\title{
FINITE ELEMENT APPROXIMATION OF SURFACTANT SPREADING ON A THIN FILM*
}

\author{
JOHN W. BARRETT ${ }^{\dagger \S}$, HARALD GARCKE ${ }^{\ddagger}$ AND ROBERT NÜRNBERG ฯ $^{-1}$
}

Abstract. We consider a fully practical finite element approximation of the following system of nonlinear degenerate parabolic equations:

$$
\begin{aligned}
\frac{\partial u}{\partial t}+\frac{1}{2} \nabla \cdot\left(u^{2} \nabla[\sigma(v)]\right)-\frac{1}{3} \nabla \cdot\left(u^{3} \nabla w\right) & =0, \quad w=-c \Delta u+a u^{-3}-\delta u^{-\nu}, \\
\frac{\partial v}{\partial t}+\nabla \cdot(u v \nabla[\sigma(v)])-\rho \Delta v-\frac{1}{2} \nabla \cdot\left(u^{2} v \nabla w\right) & =0 .
\end{aligned}
$$

The above models a surfactant-driven thin film flow in the presence of both attractive, $a>0$, and repulsive, $\delta>0$ with $\nu>3$, van der Waals forces, where $u$ is the height of the film, $v$ is the concentration of the insoluble surfactant monolayer, and $\sigma(v):=1-v$ is the typical surface tension. Here $\rho \geq 0$ and $c>0$ are the inverses of the surface Peclet number and the modified capillary number. In addition to showing stability bounds for our approximation, we prove convergence in one space dimension when $\rho>0$ and either $a=\delta=0$ or $\delta>0$. Furthermore, iterative schemes for solving the resulting nonlinear discrete system are discussed. Finally, some numerical experiments are presented.

Key words. thin film flow, surfactant, fourth order degenerate parabolic system, finite elements, convergence analysis

AMS subject classifications. 65M60, 65M12, 35K55, 35K65, 35K35, 76A20, 76D08

DOI. $10.1137 /$ S003614290139799X

1. Introduction. The study of the motion of surfactants placed on a thin layer of a viscous fluid is motivated by applications ranging from the medical treatment of premature infants to industrial coating and drying processes (cf. [13, 18, 24, 26, $27]$ ). We are interested in situations in which a free surface of a thin film contains a monolayer of a surfactant, which is a chemical that lowers the surface tension. Surface tension gradients then lead to shear stresses which force the liquid to flow toward regions of higher surface tension (Marangoni effect). In total, the liquid flow is driven by capillarity and surfactant gradient induced convection (Marangoni convection).

We consider a situation in which the thin layer of a viscous fluid spreads on a horizontal planar surface. The evolution then can be described by a free boundary problem for the Navier-Stokes equations coupled to a convection-diffusion equation for the surfactant, where the latter equation has to be solved only on the free surface. Starting from this complicated free boundary problem, it is possible, under appropriate assumptions, to use lubrication theory to derive a coupled set of nonlinear partial differential equations for the two unknowns: film thickness and surfactant concentration. It is the goal of this paper to develop and analyze a finite element method for this set of equations.

${ }^{*}$ Received by the editors November 13, 2001; accepted for publication (in revised form) February 17, 2003; published electronically September 9, 2003.

http://www.siam.org/journals/sinum/41-4/39799.html

${ }^{\dagger}$ Department of Mathematics, Imperial College, London, SW7 2AZ, UK (j.barrett@ic.ac.uk).

$¥$ Institut für Angewandte Mathematik, Wegelerstraße 6, 53115 Bonn, Germany. Present address: NWF I - Mathematik, Universität Regensburg, 93040 Regensburg, Germany (harald.garcke@ mathematik.uni-regensburg.de). The research of this author was partially supported by the RTN Programme Fronts-Singularities.

$\S$ The research of this author was partially supported by the DFG through the SFB256 Nichtlineare Partielle DGL.

IThe research of this author was supported by the EPSRC, and by the DAAD through a Doktorandenstipendium. 
Denoting by $u$ the height of the film, by $w$ the pressure, and by $v$ the concentration of the insoluble surfactant, the governing equations that one derives from lubrication theory (cf. $[13,18,31])$ are

$$
\begin{aligned}
\frac{\partial u}{\partial t}+\frac{1}{2} \nabla \cdot\left(u^{2} \nabla[\sigma(v)]\right)-\frac{1}{3} \nabla \cdot\left(u^{3} \nabla w\right) & =0, \quad w=-c \Delta u, \\
\frac{\partial v}{\partial t}+\nabla \cdot(u v \nabla[\sigma(v)])-\rho \Delta v-\frac{1}{2} \nabla \cdot\left(u^{2} v \nabla w\right) & =0 .
\end{aligned}
$$

In the following, we will denote by the vector $x$ the horizontal variables and by $y \in \mathbb{R}$ the vertical variable. The functions $u, v$, and $w$ are functions of $x$ and the time $t$, and all spatial operators like $\nabla, \nabla$., and $\Delta$ in this paper act on the horizontal variables only. The given data are $\rho \in \mathbb{R}_{\geq 0}$, the inverse of the surface Peclet number, and $c \in \mathbb{R}_{>0}$, the inverse of a modified capillary number. In addition, $\sigma:[0,1] \rightarrow[0,1]$ is the constitutive equation of state relating the surface tension $\sigma$ to $v$, e.g.,

$$
\sigma(s):=(\beta+1)[1+\theta(\beta) s]^{-3}-\beta, \quad \text { where } \quad \theta(\beta):=\left(\frac{\beta+1}{\beta}\right)^{\frac{1}{3}}-1,
$$

and $\beta \in \mathbb{R}_{>0}$ relates to the activity of the surfactant (cf. [24, p. 262]). It is reasonable to assume throughout that $\sigma$ is a monotonically decreasing function of $v$, as the surfactant lowers surface tension.

Let us now discuss some properties of the system which are important for later developments. First, we want to show how one can recover the pressure and velocity in the fluid if one knows a solution $\{u, v, w\}$ of $(1.1 \mathrm{a}, \mathrm{b})$. In lubrication theory (see [19]), it turns out that the pressure in the fluid is independent of the vertical variable $y$, and we obtain that $p(x, y, t) \equiv w(x, t)=-c \Delta u(x, t)$, where the right-hand side is an approximation to the mean curvature of the air/liquid interface. We remark that one obtains this identity from the leading order equation in lubrication theory. Another important quantity in lubrication theory is the horizontal component of the velocity, $\overrightarrow{\mathbf{V}}_{H}$, which can be computed from $\{u(x, t), v(x, t), w(x, t)\}$ as follows:

$$
\overrightarrow{\mathbf{V}}_{H}(x, y, t)=y \nabla[\sigma(v)]+\left(\frac{1}{2} y^{2}-y u\right) \nabla w,
$$

where a no-slip condition has been assumed at $y=0$. One notices that $\overrightarrow{\mathbf{V}}_{H}$ is quadratic in the $y$-direction. Furthermore, the fluid is driven by two effects: namely, by pressure gradients due to capillarity effects, $-c \nabla(\Delta u)$, and by surface tension gradients, $\nabla[\sigma(v)]$. Equation (1.3) evaluated for $y=u(x, t)$ yields the horizontal velocity on the free surface, and hence the equation for the surfactant concentration, (1.1b), can be rewritten as

$$
\frac{\partial v}{\partial t}+\nabla \cdot\left(v \overrightarrow{\mathbf{V}}_{H}(x, u(x, t), t)\right)=\rho \Delta v,
$$

which shows that it can be interpreted as a convection-diffusion equation, where the surfactant is transported with the velocity of the fluid. In addition, the equation for the film height can be expressed with the help of the fluid velocity. A straightforward computation starting from (1.1a) shows that the change of height of the film is given in terms of the horizontal component of the velocity as follows:

$$
\frac{\partial u}{\partial t}=-\nabla \cdot\left(\int_{0}^{u(x, t)} \overrightarrow{\mathbf{V}}_{H}(x, y, t) \mathrm{d} y\right) .
$$

A basic ingredient of our approach is an energy estimate for surfactant-driven flows. To derive an energy estimate involving a density function $F(v)$, we use some 
ideas from thermodynamics. First, we relate $F$ to $\sigma$ by the Gibbs identity

$$
\sigma(v)=F(v)-v F^{\prime}(v) \quad \Rightarrow \quad \sigma^{\prime}(v)=-v F^{\prime \prime}(v) .
$$

Knowing $\sigma$, the above identity determines $F$ up to a linear term. Assuming appropriate boundary conditions, which will be specified later on, one can derive an energy estimate for the surfactant-driven thin film system as

$$
\frac{\mathrm{d}}{\mathrm{d} t} \int_{\Omega}\left[\frac{c}{2}|\nabla u|^{2}+F(v)\right] \mathrm{d} x+\int_{\Omega} \int_{0}^{u}\left|\partial_{y} \overrightarrow{\mathbf{V}}_{H}\right|^{2} \mathrm{~d} y \mathrm{~d} x+\rho \int_{\Omega} F^{\prime \prime}(v)|\nabla v|^{2} \mathrm{~d} x=0
$$

where $\Omega$ is a bounded domain in $\mathbb{R}^{d}, d=1$ or 2 . To derive the above, we have used the identity

$$
\int_{0}^{u}\left|\partial_{y} \overrightarrow{\mathbf{V}}_{H}\right|^{2} \mathrm{~d} y=u|\nabla[\sigma(v)]|^{2}-u^{2} \nabla[\sigma(v)] . \nabla w+\frac{1}{3} u^{3}|\nabla w|^{2} .
$$

The identity (1.5) directly corresponds to the energy estimate for the free boundary value problem for the Navier-Stokes equations. The term $\frac{\mathrm{d}}{\mathrm{d} t} \int_{\Omega}\left[\frac{c}{2}|\nabla u|^{2}+F(v)\right] \mathrm{d} x$ describes the rate of change of energy in time, and, since one neglects inertia effects, only capillarity terms appear in the energy. The two remaining terms in (1.5) represent energy dissipation due to friction in the fluid and diffusion of the surfactant, respectively.

On recalling that $\sigma$ is monotonically decreasing, we deduce from (1.4) that $F^{\prime \prime}$ is nonnegative, and hence the identity (1.5) shows that the energy decreases in time. This energy estimate will lead to important a priori estimates. In particular, the identity (1.6) together with the inequality

$$
u^{2} \nabla[\sigma(v)] . \nabla w \leq \frac{\gamma}{2} u|\nabla[\sigma(v)]|^{2}+\frac{1}{2 \gamma} u^{3}|\nabla w|^{2}, \quad \gamma \in\left(\frac{3}{2}, 2\right),
$$

then shows that we can control $u|\nabla[\sigma(v)]|^{2}$ and $u^{3}|\nabla w|^{2}$ with the help of the energy estimate (1.5).

It is the goal of this paper to derive a finite element method that is consistent with the energy estimate (1.5); i.e., we want to derive a method that satisfies a discrete analogue.

To conclude the system, we need to specify initial and boundary conditions for $(1.1 \mathrm{a}-\mathrm{c})$. One possibility that leads to the above energy estimate would be to describe periodic boundary conditions. Instead we prescribe the following conditions at the horizontal boundary: a no-penetration condition for the velocity, a $90^{\circ}$ angle condition for the film height, and a no-flux condition for the surfactant concentration. This implies the following conditions for $x \in \partial \Omega$ and $0 \leq y \leq u(x, t)$ :

$$
\begin{array}{crr}
\nu_{\partial \Omega} \cdot \overrightarrow{\mathbf{V}}_{H}(x, y, t) \equiv \nu_{\partial \Omega} \cdot\left(y \nabla[\sigma(v)]+\left(\frac{1}{2} y^{2}-y u\right) \nabla w\right)=0, & \frac{\partial u}{\partial \nu \partial \Omega}=0, \\
\nu_{\partial \Omega} \cdot\left(v \overrightarrow{\mathbf{V}}_{H}(x, u(x, t), t)-\rho \nabla v\right) \equiv \nu_{\partial \Omega} \cdot\left(v\left(u \nabla[\sigma(v)]-\frac{1}{2} u^{2} \nabla w\right)-\rho \nabla v\right)=0,
\end{array}
$$

where $\nu_{\partial \Omega}$ is normal to $\partial \Omega$, the Lipschitz boundary of $\Omega$. Integrating the first equation in $(1.8 \mathrm{a})$ with respect to $y$ yields that

$$
\frac{1}{2} u^{2} \frac{\partial[\sigma(v)]}{\partial \nu \partial \Omega}-\frac{1}{3} u^{3} \frac{\partial w}{\partial \nu_{\partial} \Omega}=0 \quad \text { on } \partial \Omega,
$$

which means that the height averaged normal component of the horizontal velocity is zero on the boundary. Note that in the case that either $\rho>0$ or $v \sigma^{\prime}(v) \neq 0$, it can 
be seen that the first boundary condition in (1.8a) and (1.8b) are equivalent to (1.9) and (1.8b) (observe that (1.8a) holds for all $y \in[0, u(x, t)], x \in \partial \Omega$ ).

In what follows, we will therefore specify the boundary conditions

$$
\frac{1}{2} u^{2} \frac{\partial[\sigma(v)]}{\partial \nu_{\partial \Omega}}-\frac{1}{3} u^{3} \frac{\partial w}{\partial \nu_{\partial \Omega}}=\frac{\partial u}{\partial \nu_{\partial \Omega}}=u v \frac{\partial[\sigma(v)]}{\partial \nu_{\partial \Omega}}-\frac{1}{2} u^{2} v \frac{\partial w}{\partial \nu_{\partial \Omega}}-\rho \frac{\partial v}{\partial \nu_{\partial \Omega}}=0 \quad \text { on } \partial \Omega .
$$

We remark that if either $u \rho>0$ or $-u v \sigma^{\prime}(v)>0$ on $\partial \Omega$, these boundary conditions are equivalent to $\frac{\partial u}{\partial \nu_{\partial \Omega}}=\frac{\partial w}{\partial \nu_{\partial \Omega}}=\frac{\partial v}{\partial \nu_{\partial \Omega}}=0$ on $\partial \Omega$.

When surfactant is placed on the film, a thinning effect can be observed (see the numerical results in section 5). If the film thickness is in the range of a few hundred Angstroms, then molecular effects due to van der Waals forces become important. If van der Waals forces are included, an additional conservative body force enters the Navier-Stokes equations (see, e.g., [28]). In the thin film equations and in all the formulae above, the pressure $w$, related to the height $u$ previously by (1.1a), is then replaced by the reduced pressure

$$
w=-c \Delta u+\phi(u), \quad \text { where } \quad \phi(u):=a u^{-3}-\delta u^{-\nu}, \quad \nu>3 .
$$

Here $a \in \mathbb{R}_{\geq 0}$ is the scaled dimensionless Hamaker constant and $\delta \in \mathbb{R}_{\geq 0}$ represents the effect of repulsive van der Waals forces; see, e.g., [28]. When van der Waals forces are included, the energy estimate (1.5) is replaced by

$$
\frac{\mathrm{d}}{\mathrm{d} t} \int_{\Omega}\left[\frac{c}{2}|\nabla u|^{2}+F(v)+\Phi(u)\right] \mathrm{d} x+\int_{\Omega} \int_{0}^{u}\left|\partial_{y} \overrightarrow{\mathbf{V}}_{H}\right|^{2} \mathrm{~d} y \mathrm{~d} x+\rho \int_{\Omega} F^{\prime \prime}(v)|\nabla v|^{2} \mathrm{~d} x=0,
$$

where $\Phi$ is an antiderivative of $\phi$, i.e., $\Phi^{\prime}=\phi$, and the horizontal velocity $\overrightarrow{\mathbf{V}}_{H}$ is still given by (1.3) but with $w$ now defined by (1.10).

Altogether, in this paper we consider the following initial boundary value problem.

(P) Find functions $u, w, v: \Omega \times[0, T] \rightarrow \mathbb{R}$ such that

$$
\begin{aligned}
& \frac{\partial u}{\partial t}+\frac{1}{2} \nabla \cdot\left(u^{2} \nabla[\sigma(v)]\right)-\frac{1}{3} \nabla \cdot\left(u^{3} \nabla w\right)=0 \quad \text { in } \Omega_{T}, \\
& w=-c \Delta u+\phi(u) \quad \text { in } \Omega_{T}, \text { where } u>0, \\
& \frac{\partial v}{\partial t}+\nabla \cdot(u \lambda(v) \nabla[\sigma(v)])-\rho \Delta v-\frac{1}{2} \nabla \cdot\left(u^{2} \lambda(v) \nabla w\right)=0 \text { in } \Omega_{T}, \\
& u(x, 0)=u^{0}(x), \quad v(x, 0)=v^{0}(x) \quad \forall x \in \Omega, \\
& \frac{1}{2} u^{2} \frac{\partial[\sigma(v)]}{\partial \nu \partial \Omega}-\frac{1}{3} u^{3} \frac{\partial w}{\partial \nu_{\partial} \Omega}=\frac{\partial u}{\partial \nu_{\partial} \Omega}=u \lambda(v) \frac{\partial[\sigma(v)]}{\partial \nu \partial \Omega}-\frac{1}{2} u^{2} \lambda(v) \frac{\partial w}{\partial \nu_{\partial} \Omega} \\
& -\rho \frac{\partial v}{\partial \nu_{\partial} \Omega}=0 \quad \text { on } \partial \Omega \times(0, T),
\end{aligned}
$$

where $\Omega_{T}:=\Omega \times(0, T]$ and $T>0$ is a fixed positive time. In the above, $c \in \mathbb{R}_{>0}$ and $\rho \in \mathbb{R}_{\geq 0}$ are given constants, while $\phi: \mathbb{R}_{>0} \rightarrow \mathbb{R}$ is defined as in (1.10); $u^{0}$ and $v^{0}$ are given nonnegative initial profiles (e.g., $u^{0} \equiv 1$ for a film of uniform thickness and $u^{0}$ having support $\subset \subset \Omega$ for a drop). Throughout this paper, we will restrict ourselves to the model case $\sigma(v):=1-v$, the $\beta \rightarrow \infty$ limit of (1.2). We remark that physically relevant values of $v(x, t)$ lie in the interval $[0,1]$. Noting this, it is convenient for the analysis in this paper to replace the terms $u^{i} v, i=1 \rightarrow 2$, in (1.1b) by $u^{i} \lambda(v)$, where $\lambda: \mathbb{R} \rightarrow(-\infty, 1]$ is defined as

$$
\lambda(s):=[s-1]_{-}+1, \quad \text { with } \quad[s]_{-}:=\min \{s, 0\} .
$$


As $u$ and $\lambda(v)$ can take on zero values, $(\mathrm{P})$ is a degenerate parabolic system, which is fourth order in $u$. This degeneracy makes the analysis/numerical analysis of $(\mathrm{P})$ particularly difficult. Although we have assumed a no-slip condition at $y=0$ in the derivation of $(\mathrm{P})$ (see (1.3)) the results in this paper can easily be generalized to models allowing slip (see [31]).

Let us mention some work on problems that also lead to degenerate parabolic equations of fourth order. In particular, we would like to mention work on the following topics: thin film flow (cf. $[9,19,10,8,11]$ ), the Cahn-Hilliard equation with a degenerate mobility (cf. $[14,16,17])$, and models that describe dislocation densities in plasticity (cf. [20]). An existence result for the system (P) studied in this paper has been given by Wieland [31] in the case of one space dimension.

Problem (P) with $v^{0} \equiv 0$ and $\phi \equiv 0$ collapses to the thin film equation, i.e., a degenerate parabolic equation of fourth order. Degenerate parabolic equations of higher order exhibit some new characteristic features which are fundamentally different from those for second order degenerate parabolic equations such as the porous medium equation $\frac{\partial u}{\partial t}-\nabla \cdot\left(|u|^{\alpha} \nabla u\right)=0$ for a given $\alpha \in \mathbb{R}_{>0}$. The key point is that there is no maximum or comparison principle for parabolic equations of higher order. This drastically complicates the analysis since many results which are known for second order equations are proven with the help of comparison techniques. Related to this is the fact that there is no uniqueness result known for the thin film equation. Although there is no comparison principle, one of the main features of the thin film equation is the fact that one can show existence of nonnegative solutions if given nonnegative initial data. This is in contrast to linear parabolic equations of fourth order, where solutions which are initially positive may become negative in certain regions.

There is very little work on the numerical analysis of degenerate parabolic equations of fourth order; for work on thin film flows in the absence of surfactants, see $[4,32,22,21]$ and for work on degenerate Cahn-Hilliard systems, see $[5,6,3]$.

This paper is organized as follows. In section 2 we formulate a fully practical finite element approximation of problem $(\mathrm{P})$. On the discrete level, the nonnegativity of the approximation to $u$ is not guaranteed when we discretize the system $(1.12 \mathrm{a}-\mathrm{e})$ in a naive way. Following [4], we impose the nonnegativity of the discrete approximation to $u$ as a constraint and require (1.12b) only where $u$ is positive. In addition, in order to derive a discrete analogue of the energy estimate (1.11), we adapt a technique introduced in [32] and [22] for deriving a discrete entropy bound for the thin film equation.

We can derive stability bounds in space dimensions $d=1$ and 2, but we are only able to show convergence in one space dimension. This is due to the fact that the a priori bounds we derive guarantee in one space dimension only that the discrete approximation to $u$ is uniformly bounded and equicontinuous, which is necessary to be able to pass to the limit in the discrete problem. For similar reasons, the results in $[9,4,22,5,6,3]$ were restricted to one space dimension. This convergence is carried out in section 3. A convergence result for a finite element method of the thin film equation in two dimensions has been given recently by Grün [21]. Unfortunately a generalization of Grün's result to the problem presented in this paper does not seem to be possible in a straightforward manner. In section 4, we introduce and analyze algorithms to solve the nonlinear algebraic systems at each time level. Finally, in section 5 , we present some numerical computations in one and two space dimensions. We compare the computed discrete solutions with results published in $[24,26]$ and other papers. 
Notation and auxiliary results. We adopt the standard notation for Sobolev spaces, denoting the norm of $W^{m, q}(\Omega)(m \in \mathbb{N}, q \in[1, \infty])$ by $\|\cdot\|_{m, q}$ and the seminorm by $|\cdot|_{m, q}$. For $q=2, W^{m, 2}(\Omega)$ will be denoted by $H^{m}(\Omega)$ with the associated norm and seminorm written as, respectively, $\|\cdot\|_{m}$ and $|\cdot|_{m}$. Throughout $(\cdot, \cdot)$ denotes the standard $L^{2}$ inner product over $\Omega$ and $\langle\cdot, \cdot\rangle$ denotes the duality pairing between $\left(H^{1}(\Omega)\right)^{\prime}$ and $H^{1}(\Omega)$. In addition we define

$$
f \eta:=\frac{1}{\underline{m}(\Omega)}(\eta, 1) \quad \forall \eta \in L^{1}(\Omega), \quad \text { where } \underline{m}(\Omega) \text { is the measure of } \Omega .
$$

We require also the Hölder space $C_{x, t}^{p_{1}, p_{2}}\left(\bar{\Omega}_{T}\right)$ for $p_{i} \in(0,1]$, which denotes those functions whose time (spatial) derivative(s) is (are) Hölder continuous over $\bar{\Omega}_{T}$ with exponent $p_{1}\left(p_{2}\right)$.

For later purposes, we recall the following well-known Sobolev interpolation result (see, e.g., [1]): Let $m \geq 1, q \in\left(\frac{d}{m}, \infty\right], r \in[q, \infty]$, and $\mu:=\frac{d}{m}\left(\frac{1}{q}-\frac{1}{r}\right)$. Then there is a constant $C$ depending only on $\Omega, m, q, r$ such that

$$
|z|_{0, r} \leq C|z|_{0, q}^{1-\mu}\|z\|_{m, q}^{\mu} \quad \forall z \in W^{m, q}(\Omega) .
$$

It is convenient to introduce the "inverse Laplacian" operator $\mathcal{G}: \mathcal{F} \rightarrow Z$ such that

$$
(\nabla \mathcal{G} z, \nabla \eta)=\langle z, \eta\rangle \quad \forall \eta \in H^{1}(\Omega),
$$

where $\mathcal{F}:=\left\{z \in\left(H^{1}(\Omega)\right)^{\prime}:\langle z, 1\rangle=0\right\}$ and $Z:=\left\{z \in H^{1}(\Omega):(z, 1)=0\right\}$. The wellposedness of $\mathcal{G}$ follows from the Lax-Milgram theorem and the Poincaré inequality

$$
|\eta|_{0, q} \leq C\left(|\eta|_{1, q}+|(\eta, 1)|\right) \quad \forall \eta \in W^{1, q}(\Omega) \quad \text { and } \quad q \in[1, \infty] .
$$

One can define a norm on $\mathcal{F}$ by

$$
\|z\|_{-1}:=|\mathcal{G} z|_{1}=\langle z, \mathcal{G} z\rangle^{\frac{1}{2}} \quad \forall z \in \mathcal{F} .
$$

We note also for future reference that using Young's inequality

$$
r s \leq \frac{\gamma}{2} r^{2}+\frac{1}{2 \gamma} s^{2} \quad \forall r, s \in \mathbb{R}, \gamma \in \mathbb{R}_{>0}
$$

yields for all $\gamma \in \mathbb{R}_{>0}$ that

$$
\langle z, \eta\rangle=(\nabla \mathcal{G} z, \nabla \eta) \leq\|z\|_{-1}|\eta|_{1} \leq \frac{\gamma}{2}|\eta|_{1}^{2}+\frac{1}{2 \gamma}\|z\|_{-1}^{2} \quad \forall z \in \mathcal{F}, \eta \in H^{1}(\Omega) .
$$

Throughout $C$ denotes a generic constant independent of $h, \tau$, and $\varepsilon$, the mesh and temporal discretization parameters and the regularization parameter. In addition, $C\left(a_{1}, \ldots, a_{I}\right)$ denotes a constant depending on the arguments $\left\{a_{i}\right\}_{i=1}^{I}$.

2. Finite element approximation. We consider the finite element approximation of $(\mathrm{P})$ at first under the following assumptions on the mesh:

(A) Let $\Omega$ be a polygonal domain if $d=2$. Let $\left\{\mathcal{T}^{h}\right\}_{h>0}$ be a quasi-uniform family of partitionings of $\Omega$ into disjoint open simplices $\kappa$ with $h_{\kappa}:=\operatorname{diam}(\kappa)$ and $h:=\max _{\kappa \in \mathcal{T}^{h}} h_{\kappa}$ so that $\bar{\Omega}=\cup_{\kappa \in \mathcal{T}^{h}} \bar{\kappa}$. In addition, it is assumed for $d=2$ that all simplices $\kappa \in \mathcal{T}^{h}$ are right-angled.

We note that the quasi uniformity assumption can be avoided at the expense of a mild constraint on the minimum time step; see Remark 3.5 below. Furthermore we 
note that the right-angled simplices assumption is not a severe constraint, as there exist adaptive finite element codes that satisfy this requirement; see, e.g., [30].

Associated with $\mathcal{T}^{h}$ is the finite element space $S^{h}:=\left\{\chi \in C(\bar{\Omega}):\left.\chi\right|_{\kappa}\right.$ is linear for all $\left.\kappa \in \mathcal{T}^{h}\right\} \subset H^{1}(\Omega)$. We introduce also $K^{h}:=\left\{\chi \in S^{h}: \chi \geq 0\right.$ in $\left.\Omega\right\} \subset K$, where $K:=\left\{\eta \in H^{1}(\Omega): \eta \geq 0\right.$ a.e. in $\left.\Omega\right\}$. Let $J$ be the set of nodes of $\mathcal{T}^{h}$ and $\left\{p_{j}\right\}_{j \in J}$ the coordinates of these nodes. Let $\left\{\chi_{j}\right\}_{j \in J}$ be the standard basis functions for $S^{h}$; that is, $\chi_{j} \in K^{h}$ and $\chi_{j}\left(p_{i}\right)=\delta_{i j}$ for all $i, j \in J$. We introduce $\pi^{h}: C(\bar{\Omega}) \rightarrow S^{h}$, the interpolation operator, such that $\left(\pi^{h} \eta\right)\left(p_{j}\right)=\eta\left(p_{j}\right)$ for all $j \in J$. A discrete semi-inner product on $C(\bar{\Omega})$ is then defined by

$$
\left(\eta_{1}, \eta_{2}\right)^{h}:=\int_{\Omega} \pi^{h}\left(\eta_{1}(x) \eta_{2}(x)\right) \mathrm{d} x=\sum_{j \in J} m_{j} \eta_{1}\left(p_{j}\right) \eta_{2}\left(p_{j}\right)
$$

where $m_{j}:=\left(1, \chi_{j}\right)>0$. The induced discrete seminorm is then $|\eta|_{h}:=\left[(\eta, \eta)^{h}\right]^{\frac{1}{2}}$, where $\eta \in C(\bar{\Omega})$. We introduce also the $L^{2}$ projection $Q^{h}: L^{2}(\Omega) \rightarrow S^{h}$ defined by

$$
\left(Q^{h} \eta, \chi\right)^{h}=(\eta, \chi) \quad \forall \chi \in S^{h} .
$$

In this paper, for simplicity, we consider only the model case when the surface tension is given by $\sigma(s):=1-s$, which is the limit as $\beta \rightarrow \infty$ in (1.2) and is commonly used in the physics/engineering literature (cf. [27]). On recalling (1.4), we then define a function $F$ such that $v \nabla\left[F^{\prime}(v)\right]=-\nabla[\sigma(v)]$; that is, $F^{\prime \prime}(s)=-s^{-1} \sigma^{\prime}(s)=s^{-1} \Rightarrow$ $F(s)=s(\ln s-1)+1$. For computational purposes, we replace $F \in C^{\infty}\left(\mathbb{R}_{>0}\right)$ for any $\varepsilon \in(0,1)$ by the regularized function $F_{\varepsilon}: \mathbb{R} \rightarrow \mathbb{R}_{\geq 0}$ such that

$$
F_{\varepsilon}(s):= \begin{cases}\frac{s^{2}-\varepsilon^{2}}{2 \varepsilon}+(\ln \varepsilon-1) s+1, & s \leq \varepsilon \\ s(\ln s-1)+1, & \varepsilon \leq s \leq 1 \\ \frac{1}{2}(s-1)^{2}, & 1 \leq s\end{cases}
$$

Hence $F_{\varepsilon} \in C^{2,1}(\mathbb{R})$ with the first two derivatives of $F_{\varepsilon}$ given by

$$
F_{\varepsilon}^{\prime}(s):=\left\{\begin{array}{ll}
\varepsilon^{-1} s+\ln \varepsilon-1, & s \leq \varepsilon, \\
\ln s, & \varepsilon \leq s \leq 1, \\
s-1, & 1 \leq s,
\end{array} \quad \text { and } \quad F_{\varepsilon}^{\prime \prime}(s):= \begin{cases}\varepsilon^{-1}, & s \leq \varepsilon, \\
s^{-1}, & \varepsilon \leq s \leq 1, \\
1, & 1 \leq s\end{cases}\right.
$$

respectively. For later purposes, we note that

$$
F_{\varepsilon}(s) \geq \frac{s^{2}}{4}-\frac{1}{2} \quad \forall s \geq 0 \quad \text { and } \quad F_{\varepsilon}(s) \geq \frac{s^{2}}{2 \varepsilon} \quad \forall s \leq 0 .
$$

This holds since

$$
\begin{array}{rlrl}
F_{\varepsilon}(s):=\frac{1}{2}(s-1)^{2} \geq \frac{1}{2}(s-1)^{2}-\left(\frac{1}{2} s-1\right)^{2} & =\frac{s^{2}}{4}-\frac{1}{2} & & \text { if } s \geq 1, \\
F_{\varepsilon}^{\prime}(s) \leq 0 \quad \Rightarrow \quad F_{\varepsilon}(s) \geq F_{\varepsilon}(1)=0 \geq \frac{s^{2}}{4}-\frac{1}{2} & & \text { if } s \in[0,1], \\
F_{\varepsilon}(s):=\frac{s^{2}-\varepsilon^{2}}{2 \varepsilon}+(\ln \varepsilon-1) s+1 \geq \frac{s^{2}}{2 \varepsilon}+\left(1-\frac{\varepsilon}{2}\right) \geq \frac{s^{2}}{2 \varepsilon} & & \text { if } s \leq 0 .
\end{array}
$$

Similarly to the approach in [32] and [22], we introduce $\Lambda_{\varepsilon}: S^{h} \rightarrow\left[L^{\infty}(\Omega)\right]^{d \times d}$ such that for all $z^{h} \in S^{h}$ and a.e. in $\Omega$,

$\Lambda_{\varepsilon}\left(z^{h}\right)$ is symmetric and positive semidefinite and $\Lambda_{\varepsilon}\left(z^{h}\right) \nabla \pi^{h}\left[F_{\varepsilon}^{\prime}\left(z^{h}\right)\right]=\nabla z^{h}$. 
Following [22], we now give the construction of $\Lambda_{\varepsilon}$. Let $\left\{e_{i}\right\}_{i=1}^{d}$ be the orthonormal vectors in $\mathbb{R}^{d}$ such that the $j$ th component of $e_{i}$ is $\delta_{i j}, i, j=1 \rightarrow d$. Given nonzero constants $\alpha_{i}, i=1 \rightarrow d$, let $\widehat{\kappa}\left(\left\{\alpha_{i}\right\}_{i=1}^{d}\right)$ be the reference open simplex in $\mathbb{R}^{d}$ with vertices $\left\{\widehat{p}_{i}\right\}_{i=0}^{d}$, where $\widehat{p}_{0}$ is the origin and $\widehat{p}_{i}=\alpha_{i} e_{i}, i=1 \rightarrow d$. Given a $\kappa \in \mathcal{T}^{h}$ with vertices $\left\{p_{j_{i}}\right\}_{i=0}^{d}$ such that $p_{j_{0}}$ is the right-angled vertex, there exist a rotation matrix $R_{\kappa}$ and nonzero constants $\left\{\alpha_{i}\right\}_{i=1}^{d}$ such that the mapping $\mathcal{R}_{\kappa}: \widehat{x} \in \mathbb{R}^{d} \rightarrow$ $p_{j_{0}}+R_{\kappa} \widehat{x} \in \mathbb{R}^{d}$ maps the vertex $\widehat{p}_{i}$ to $p_{j_{i}}, i=0 \rightarrow d$, and hence $\widehat{\kappa} \equiv \widehat{\kappa}\left(\left\{\alpha_{i}\right\}_{i=1}^{d}\right)$ to $\kappa$. For any $z^{h} \in S^{h}$, we then set

$$
\left.\Lambda_{\varepsilon}\left(z^{h}\right)\right|_{\kappa}:=\left.R_{\kappa} \widehat{\Lambda}_{\varepsilon}\left(\widehat{z}^{h}\right)\right|_{\widehat{\kappa}} R_{\kappa}^{T},
$$

where $\widehat{z}^{h}(\widehat{x}) \equiv z^{h}\left(\mathcal{R}_{\kappa} \widehat{x}\right)$ for all $\widehat{x} \in \bar{\kappa}$ and $\left.\widehat{\Lambda}_{\varepsilon}\left(\widehat{z}^{h}\right)\right|_{\widehat{\kappa}}$ is the $d \times d$ diagonal matrix with diagonal entries, $k=1 \rightarrow d$,

$$
\left.\left[\widehat{\Lambda}_{\varepsilon}\left(\widehat{z}^{h}\right)\right]_{k k}\right|_{\widehat{\kappa}}:= \begin{cases}\frac{\widehat{z}^{h}\left(\widehat{p}_{k}\right)-\widehat{z}^{h}\left(\widehat{p}_{0}\right)}{F_{\varepsilon}^{\prime}\left(\widehat{z}^{h}\left(\widehat{p}_{k}\right)\right)-F_{\varepsilon}^{\prime}\left(\widehat{z}^{h}\left(\widehat{p}_{0}\right)\right)} \equiv \frac{z^{h}\left(p_{j_{k}}\right)-z^{h}\left(p_{j_{0}}\right)}{F_{\varepsilon}^{\prime}\left(z^{h}\left(p_{j_{k}}\right)\right)-F_{\varepsilon}^{\prime}\left(z^{h}\left(p_{j_{0}}\right)\right)} & \text { if } z^{h}\left(p_{j_{k}}\right) \neq z^{h}\left(p_{j_{0}}\right), \\ \frac{1}{F_{\varepsilon}^{\prime \prime}\left(\widehat{z}^{h}\left(\widehat{p}_{0}\right)\right)} \equiv \frac{1}{F_{\varepsilon}^{\prime \prime}\left(z^{h}\left(p_{j_{0}}\right)\right)} & \text { if } z^{h}\left(p_{j_{k}}\right)=z^{h}\left(p_{j_{0}}\right) .\end{cases}
$$

As $R_{\kappa}^{T} \equiv R_{\kappa}^{-1}, \nabla z^{h} \equiv R_{\kappa} \widehat{\nabla} \widehat{z}^{h}$, where $x \equiv\left(x_{1}, \ldots, x_{d}\right)^{T}, \nabla \equiv\left(\frac{\partial}{\partial x_{1}}, \ldots, \frac{\partial}{\partial x_{d}}\right)^{T}$, $\widehat{x} \equiv\left(\widehat{x}_{1}, \ldots, \widehat{x}_{d}\right)^{T}$, and $\widehat{\nabla} \equiv\left(\frac{\partial}{\partial \widehat{x}_{1}}, \ldots, \frac{\partial}{\partial \widehat{x}_{d}}\right)^{T}$, it easily follows that $\Lambda_{\varepsilon}\left(z^{h}\right)$ constructed in (2.6) and (2.7) satisfies (2.5). It is this construction that requires the right angle constraint on the partitioning $\mathcal{T}^{h}$. Furthermore, we note from (2.3) that for all $\kappa \in \mathcal{T}^{h}$,

$$
\begin{aligned}
\left.\nabla z^{h} \cdot \nabla \pi^{h}\left[F_{\varepsilon}^{\prime}\left(z^{h}\right)\right]\right|_{\kappa} & \left.\equiv R_{\kappa} \widehat{\nabla} \widehat{z}^{h} \cdot R_{\kappa} \widehat{\nabla} \widehat{\pi}^{h}\left[F_{\varepsilon}^{\prime}\left(\widehat{z}^{h}\right)\right]\right|_{\widehat{\kappa}} \\
& \left.\equiv \widehat{\nabla} \widehat{z}^{h} \cdot \widehat{\nabla} \widehat{\pi}^{h}\left[F_{\varepsilon}^{\prime}\left(\widehat{z}^{h}\right)\right]\right|_{\widehat{\kappa}} \geq\left.\left.\left|\widehat{\nabla} \widehat{z}^{h}\right|^{2}\right|_{\widehat{\kappa}} \equiv\left|\nabla z^{h}\right|^{2}\right|_{\kappa} \\
\Longrightarrow \quad\left(\nabla z^{h}, \nabla \pi^{h}\left[F_{\varepsilon}^{\prime}\left(z^{h}\right)\right]\right) & \geq\left|z^{h}\right|_{1}^{2} \quad \forall z^{h} \in S^{h},
\end{aligned}
$$

where $\left(\widehat{\pi}^{h} \widehat{\eta}\right)(\widehat{x}) \equiv\left(\pi^{h} \eta\right)\left(\mathcal{R}_{\kappa} \widehat{x}\right)$ and $\widehat{\eta}(\widehat{x}) \equiv \eta\left(\mathcal{R}_{\kappa} \widehat{x}\right)$ for all $\widehat{x} \in \bar{\kappa}$.

To define our approximation of (P), it is convenient to split $\Phi$ (recall (1.11)) into its convex and concave parts. We have for given $a, \delta \in \mathbb{R}_{\geq 0}$, and $\nu>3$ that for all $s \in \mathbb{R}_{>0}$

$$
\begin{array}{lll}
\Phi(s)=\Phi^{+}(s)+\Phi^{-}(s), & \text { where } & \Phi^{+}(s):=\frac{\delta}{\nu-1} s^{1-\nu}, \quad \Phi^{-}(s):=-\frac{a}{2} s^{-2}, \\
\phi(s)=\Phi^{\prime}(s)=\phi^{+}(s)+\phi^{-}(s), & \text { where } \quad \phi^{+}(s):=\left(\Phi^{+}\right)^{\prime}(s)=-\delta s^{-\nu}, \\
& \phi^{-}(s):=\left(\Phi^{-}\right)^{\prime}(s)=a s^{-3} .
\end{array}
$$

For future reference, we note that the following hold for all $s \in \mathbb{R}_{>0}$ :

$$
\Phi(s) \geq \Phi\left(\left(\frac{\delta}{a}\right)^{\frac{1}{\nu-3}}\right)=\frac{a(3-\nu)}{2(\nu-1)}\left(\frac{a}{\delta}\right)^{\frac{2}{\nu-3}} \text { and }\left|\Phi^{-}(s)\right| \leq \frac{a(\nu-3)}{2(\nu-1)}\left(\frac{2 a}{\delta}\right)^{\frac{2}{\nu-3}}+\frac{1}{2} \Phi^{+}(s) .
$$

In addition to $\mathcal{T}^{h}$, let $0=t_{0}<t_{1}<\cdots<t_{N-1}<t_{N}=T$ be a partitioning of $[0, T]$ into possibly variable time steps $\tau_{n}:=t_{n}-t_{n-1}, n=1 \rightarrow N$. We set $\tau:=\max _{n=1 \rightarrow N} \tau_{n}$. For any given $\varepsilon \in(0,1)$, we then consider the following fully practical finite element approximation of $(\mathrm{P})$ with $\sigma(v):=1-v$ and $\delta=0\left(\phi^{+} \equiv 0\right)$. 
$\left(\mathrm{P}_{\varepsilon}^{h, \tau}\right)$ For $n \geq 1$ find $\left\{U_{\varepsilon}^{n}, W_{\varepsilon}^{n}, V_{\varepsilon}^{n}\right\} \in K^{h} \times\left[S^{h}\right]^{2}$ such that for all $\chi \in S^{h}$, $z^{h} \in K^{h}$

$$
\begin{gathered}
\left(\frac{U_{\varepsilon}^{n}-U_{\varepsilon}^{n-1}}{\tau_{n}}, \chi\right)^{h}+\frac{1}{3}\left(\pi^{h}\left[\left(U_{\varepsilon}^{n-1}\right)^{3}\right] \nabla W_{\varepsilon}^{n}, \nabla \chi\right)=-\frac{1}{2}\left(\pi^{h}\left[\left(U_{\varepsilon}^{n-1}\right)^{2}\right] \nabla V_{\varepsilon}^{n-1}, \nabla \chi\right), \\
c\left(\nabla U_{\varepsilon}^{n}, \nabla\left(z^{h}-U_{\varepsilon}^{n}\right)\right)+\left(\phi^{-}\left(U_{\varepsilon}^{n-1}+\varepsilon\right), z^{h}-U_{\varepsilon}^{n}\right)^{h} \geq\left(W_{\varepsilon}^{n}, z^{h}-U_{\varepsilon}^{n}\right)^{h}, \\
\left(\frac{V_{\varepsilon}^{n}-V_{\varepsilon}^{n-1}}{\tau_{n}}, \chi\right)^{h}+\rho\left(\nabla V_{\varepsilon}^{n}, \nabla \chi\right)+\left(U_{\varepsilon}^{n} \Lambda_{\varepsilon}\left(V_{\varepsilon}^{n}\right) \nabla V_{\varepsilon}^{n}, \nabla \chi\right) \\
=-\frac{1}{2}\left(\pi^{h}\left[\left(U_{\varepsilon}^{n}\right)^{\frac{1}{2}}\left(U_{\varepsilon}^{n-1}\right)^{\frac{3}{2}}\right] \Lambda_{\varepsilon}\left(V_{\varepsilon}^{n}\right) \nabla W_{\varepsilon}^{n}, \nabla \chi\right),
\end{gathered}
$$

$$
\text { approximations of } u^{0} \text { and } v^{0} \text {, respectively; e.g., }
$$
$U_{\varepsilon}^{0} \equiv \pi^{h} u^{0}$ or $Q^{h} u^{0}$ and similarly $V_{\varepsilon}^{0}$.

If $a=0\left(\phi^{-} \equiv 0\right)$, then setting $V_{\varepsilon}^{n} \equiv 0, n=0 \rightarrow N,(2.11 \mathrm{a}, \mathrm{b})$ collapses to the approximation of the thin film equation, (1.12a-e) with $v \equiv 0$, studied in [4], except that there $\pi^{h}\left[\left(U_{\varepsilon}^{n-1}\right)^{3}\right]$ in (2.11a) is replaced by $\left(U_{\varepsilon}^{n-1}\right)^{3}$ and so is less practical than (2.11a). If $\delta>0\left(\phi^{+} \not \equiv 0\right)$, then $\left(\mathrm{P}_{\varepsilon}^{h, \tau}\right)$ above is modified as follows.

$\left(\mathrm{P}_{\delta, \varepsilon}^{h, \tau}\right)$ For $n \geq 1$ find $\left\{U_{\varepsilon}^{n}, W_{\varepsilon}^{n}, V_{\varepsilon}^{n}\right\} \in\left[S^{h}\right]^{3}$ such that (2.11a,c) hold with (2.11b) replaced by

$$
c\left(\nabla U_{\varepsilon}^{n}, \nabla \chi\right)+\left(\phi^{+}\left(U_{\varepsilon}^{n}\right)+\phi^{-}\left(U_{\varepsilon}^{n-1}\right), \chi\right)^{h}=\left(W_{\varepsilon}^{n}, \chi\right)^{h} \quad \forall \chi \in S^{h},
$$

where in addition it is assumed that $U_{\varepsilon}^{0}>0$.

Note that the convex (concave) terms in $\Phi$ are approximated implicitly (explicitly) in (2.11b) and (2.12). If $\delta=0$, we can guarantee only that $U_{\varepsilon}^{n-1} \geq 0$ and hence the choice of $\phi^{-}\left(U_{\varepsilon}^{n-1}+\varepsilon\right)$ instead of $\phi^{-}\left(U_{\varepsilon}^{n-1}\right)$ on the left-hand side of $(2.11 \mathrm{~b})$. Whereas if $\delta>0$ and $U_{\varepsilon}^{0}>0$, one can ensure that $\phi^{-}\left(U_{\varepsilon}^{n-1}\right)$ is well defined for $n \geq 1$; see Theorem 2.6 below.

Below we recall some well-known results concerning $S^{h}$ for any $\kappa \in \mathcal{T}^{h}, \chi, z^{h} \in$ $S^{h}, \eta_{1}, \eta_{2} \in C(\bar{\Omega})$ and for $m=0$ or 1 :

$$
\begin{aligned}
\lim _{h \rightarrow 0}\left|\left(I-\pi^{h}\right) \eta_{1}\right|_{0, \infty} & =0, \\
\int_{\kappa} \chi^{2} \mathrm{~d} x & \leq \int_{\kappa} \pi^{h}\left[\chi^{2}\right] \mathrm{d} x \leq(d+2) \int_{\kappa} \chi^{2} \mathrm{~d} x, \\
\int_{\kappa} \pi^{h}\left[\eta_{1} \eta_{2}\right] \nabla \chi \cdot \nabla z^{h} \mathrm{~d} x & \leq\left[\int_{\kappa} \pi^{h}\left[\eta_{1}^{2}\right]|\nabla \chi|^{2} \mathrm{~d} x\right]^{\frac{1}{2}}\left[\int_{\kappa} \pi^{h}\left[\eta_{2}^{2}\right]\left|\nabla z^{h}\right|^{2} \mathrm{~d} x\right]^{\frac{1}{2}}, \\
\left|\pi^{h}\left[\eta_{1} \eta_{2}\right](x)\right|^{2} & \leq\left|\pi^{h} \eta_{1}\right|_{0, \infty}^{2} \pi^{h}\left[\eta_{2}^{2}\right](x) \quad \forall x \in \bar{\Omega} ; \\
\left|\left(\chi, z^{h}\right)-\left(\chi, z^{h}\right)^{h}\right| & \leq\left|\left(I-\pi^{h}\right)\left(\chi z^{h}\right)\right|_{0,1} \leq C h^{1+m}|\chi|_{m}\left|z^{h}\right|_{1} .
\end{aligned}
$$

If $d=1$, then we have for $m=0$ or 1 that

$$
\begin{aligned}
\left|\left(I-\pi^{h}\right) \eta\right|_{m, r} & \leq C h^{1-m}|\eta|_{1, r} \quad \forall \eta \in W^{1, r}(\Omega), \quad \text { for any } r \in[1, \infty] ; \\
\lim _{h \rightarrow 0}\left\|\left(I-\pi^{h}\right) \eta\right\|_{1} & =0 \quad \forall \eta \in H^{1}(\Omega) .
\end{aligned}
$$

It follows from (2.2) that

$$
\left(Q^{h} \eta\right)\left(p_{j}\right)=\frac{\left(\eta, \chi_{j}\right)}{\left(1, \chi_{j}\right)} \quad \forall j \in J \quad \Longrightarrow \quad\left|Q^{h} \eta\right|_{0, \infty} \leq|\eta|_{0, \infty} \quad \forall \eta \in L^{\infty}(\Omega) .
$$

Finally, as we have a quasi-uniform family of partitionings, it holds for $m=0$ or 1 that

$$
\left|\left(I-Q^{h}\right) \eta\right|_{m} \leq C h^{1-m}\|\eta\|_{1} \quad \forall \eta \in H^{1}(\Omega) .
$$


We define $Z^{h}:=\left\{z^{h} \in S^{h}:\left(z^{h}, 1\right)=0\right\} \subset \mathcal{F}^{h}:=\left\{z \in C(\bar{\Omega}):(z, 1)^{h}=0\right\}$. Then, similarly to (1.16), we introduce $\mathcal{G}^{h}: \mathcal{F}^{h} \rightarrow Z^{h}$ such that

$$
\left(\nabla \mathcal{G}^{h} z, \nabla \chi\right)=(z, \chi)^{h} \quad \forall \chi \in S^{h} .
$$

It is easily established, as we have a quasi-uniform family of partitionings, that

$$
\left|z^{h}\right|_{0} \leq C h^{-1}\left|\mathcal{G} z^{h}\right|_{1} \quad \forall z^{h} \in Z^{h} .
$$

We now adapt and extend the approach in [4] to establish the existence of a solution $\left\{U_{\varepsilon}^{n}, W_{\varepsilon}^{n}, V_{\varepsilon}^{n}\right\}_{n=1}^{N}$ to $\left(\mathrm{P}_{\varepsilon}^{h, \tau}\right)$. First, we need to introduce some notation. In particular, we define sets $Z^{h}\left(U_{\varepsilon}^{n-1}\right)$ in which we seek the update $U_{\varepsilon}^{n}-U_{\varepsilon}^{n-1}$. Given $q^{h} \in K^{h}$, we set $J_{0}\left(q^{h}\right):=\left\{j \in J:\left(\pi^{h}\left[\left(q^{h}\right)^{3}\right], \chi_{j}\right)=0\right\}$. All other nodes we call active nodes, and they can be uniquely partitioned so that $J_{+}\left(q^{h}\right):=J \backslash$ $J_{0}\left(q^{h}\right)=\bigcup_{m=1}^{M} I_{m}\left(q^{h}\right), M \geq 1$, where $I_{m}\left(q^{h}\right), m=1 \rightarrow M$, are mutually disjoint and maximally connected in the following sense: $I_{m}\left(q^{h}\right)$ is said to be connected if for all $j, k \in I_{m}\left(q^{h}\right)$, there exist $\left\{\kappa_{\ell}\right\}_{\ell=1}^{L} \subseteq \mathcal{T}^{h}$, not necessarily distinct, such that

$$
p_{j} \in \bar{\kappa}_{1}, p_{k} \in \bar{\kappa}_{L} ; \quad \bar{\kappa}_{\ell} \cap \bar{\kappa}_{\ell+1} \neq \emptyset, \ell=1 \rightarrow L-1 ; \quad q^{h} \not \equiv 0 \text { on } \kappa_{\ell}, \ell=1 \rightarrow L .
$$

$I_{m}\left(q^{h}\right)$ is said to be maximally connected if there is no other connected subset of $J_{+}\left(q^{h}\right)$, which contains $I_{m}\left(q^{h}\right)$. We then set

$$
\begin{gathered}
Z^{h}\left(q^{h}\right):=\left\{z^{h} \in S^{h}: z^{h}\left(p_{j}\right)=0 \quad \forall j \in J_{0}\left(q^{h}\right) \quad\right. \text { and } \\
\left.\left(z^{h}, \Xi_{m}\left(q^{h}\right)\right)^{h}=0, m=1 \rightarrow M\right\},
\end{gathered}
$$

where $\Xi_{m}\left(q^{h}\right):=\sum_{j \in I_{m}\left(q^{h}\right)} \chi_{j}$ for $m=1 \rightarrow M$. An immediate consequence of the above definitions is that on any $\kappa \in \mathcal{T}^{h}$ either

$$
q^{h} \equiv 0 \quad \text { or } \quad \Xi_{m_{\star}}\left(q^{h}\right) \equiv 1 \text { for some } m_{\star} \text { and } \Xi_{m}\left(q^{h}\right) \equiv 0 \text { for } m \neq m_{\star} .
$$

This follows since if $q^{h} \not \equiv 0$ on $\kappa$, then all vertices of $\kappa$ belong to the set of active nodes $J_{+}\left(q^{h}\right)$. Using the fact that $I_{m}\left(q^{h}\right), m=1 \rightarrow M$, are maximally connected, we can conclude that there exists an $m_{\star}$ such that all vertices of $\kappa$ belong to $I_{m_{\star}}\left(q^{h}\right)$ and therefore, $\Xi_{m_{\star}}\left(q^{h}\right) \equiv 1$ on $\kappa$. The desired result now follows since $I_{m}\left(q^{h}\right), m=$ $1 \rightarrow M$, are mutually disjoint.

For later reference, we state that any $z^{h} \in S^{h}$ can be written as

$$
z^{h} \equiv \sum_{j \in J} z^{h}\left(p_{j}\right) \chi_{j} \equiv \bar{z}^{h}+\sum_{j \in J_{0}\left(q^{h}\right)} z^{h}\left(p_{j}\right) \chi_{j}+\sum_{m=1}^{M} \frac{\left(z^{h}, \Xi_{m}\left(q^{h}\right)\right)^{h}}{\left(1, \Xi_{m}\left(q^{h}\right)\right)} \Xi_{m}\left(q^{h}\right)
$$

where $\bar{z}^{h}:=\sum_{m=1}^{M} \sum_{j \in I_{m}\left(q^{h}\right)}\left[z^{h}\left(p_{j}\right)-\frac{\left(z^{h}, \Xi_{m}\left(q^{h}\right)\right)^{h}}{\left(1, \Xi_{m}\left(q^{h}\right)\right)}\right] \chi_{j} \in Z^{h}\left(q^{h}\right)$ is the projection with respect to the $(\cdot, \cdot)^{h}$ scalar product of $z^{h}$ onto $Z^{h}\left(q^{h}\right)$. In order to express $W_{\varepsilon}^{n}$ in terms of $U_{\varepsilon}^{n}$ and $U_{\varepsilon}^{n-1}$, we introduce for all $q^{h} \in K^{h}$ the discrete anisotropic Green's operator $\mathcal{G}_{q^{h}}^{h}: Z^{h}\left(q^{h}\right) \rightarrow Z^{h}\left(q^{h}\right)$ such that

$$
\left(\pi^{h}\left[\left(q^{h}\right)^{3}\right] \nabla \mathcal{G}_{q^{h}}^{h} z^{h}, \nabla \chi\right)=\left(z^{h}, \chi\right)^{h} \quad \forall \chi \in S^{h} .
$$

To show the well-posedness of $\mathcal{G}_{q^{h}}^{h}$, we first note that on choosing $\chi \equiv \chi_{j}, j \in J_{0}\left(q^{h}\right)$, in (2.27) leads to both sides vanishing on noting (2.24). Similarly, choosing $\chi \equiv \Xi_{m}\left(q^{h}\right)$, 
$m=1 \rightarrow M$, in (2.27) leads to both sides vanishing on noting (2.25) and (2.24). Therefore, for well-posedness, it remains to prove uniqueness as $Z^{h}\left(q^{h}\right)$ has finite dimension. If there exist two solutions $Z^{(i)}, i=1,2$, with $\left(\pi^{h}\left[\left(q^{h}\right)^{3}\right] \nabla Z^{(i)}, \nabla \chi\right)=$ $\left(z^{h}, \chi\right)^{h}$ for all $\chi \in S^{h}$, then $Z:=Z^{(2)}-Z^{(1)} \in Z^{h}\left(q^{h}\right)$ satisfies, on noting (2.25),

$$
C\left(q^{h}, h\right) \sum_{m=1}^{M} \int_{\Omega_{m}}|\nabla Z|^{2} \mathrm{~d} x \leq \sum_{m=1}^{M} \int_{\Omega_{m}} \pi^{h}\left[\left(q^{h}\right)^{3}\right]|\nabla Z|^{2} \mathrm{~d} x=\int_{\Omega} \pi^{h}\left[\left(q^{h}\right)^{3}\right]|\nabla Z|^{2} \mathrm{~d} x=0
$$

for some positive constant $C\left(q^{h}, h\right)$, where $\Omega_{m}:=\left\{\cup_{\kappa \in \mathcal{T} h} \bar{\kappa}:\left.\Xi_{m}\left(q^{h}\right)\right|_{\kappa} \equiv 1\right\}$. Hence it follows that $Z$ is constant on each $\Omega_{m}$. However, as $Z \in Z^{h}\left(q^{h}\right)$, it follows that $Z \equiv 0$. Finally, note that $Z^{h}\left(q^{h}\right) \subseteq Z^{h}$ for all $q^{h} \in K^{h}$ and in addition that $Z^{h}\left(q^{h}\right)$ defined in (2.24) is equal to $Z^{h}$ if $q^{h}$ is strictly positive.

LEMma 2.1. Let the assumptions (A) hold, and let $\|\cdot\|$ denote the spectral norm on $\mathbb{R}^{d \times d}$. Then for any given $\varepsilon \in(0,1)$, the function $\Lambda_{\varepsilon}: S^{h} \rightarrow\left[L^{\infty}(\Omega)\right]^{d \times d}$ satisfies

$$
\varepsilon \xi^{T} \xi \leq \xi^{T} \Lambda_{\varepsilon}\left(z^{h}\right) \xi \leq \xi^{T} \xi \quad \forall \xi \in \mathbb{R}^{d}, \quad \forall z^{h} \in S^{h}
$$

and is continuous. In particular, it holds for all $z_{1}^{h}, z_{2}^{h} \in S^{h}, \kappa \in \mathcal{T}^{h}$ that

$$
\begin{aligned}
& \left\|\left.\left[\Lambda_{\varepsilon}\left(z_{1}^{h}\right)-\Lambda_{\varepsilon}\left(z_{2}^{h}\right)\right]\right|_{\kappa}\right\|=\left\|\left.\left[\widehat{\Lambda}_{\varepsilon}\left(\widehat{z}_{1}^{h}\right)-\widehat{\Lambda}_{\varepsilon}\left(\widehat{z}_{2}^{h}\right)\right]\right|_{\kappa}\right\| \\
& \quad \leq \max _{s \in \mathbb{R}} F_{\varepsilon}^{\prime \prime}(s) \max _{s \in \mathbb{R}}\left[F_{\varepsilon}^{\prime \prime}(s)\right]^{-1} \max _{k=1 \rightarrow d}\left[\left|z_{1}^{h}\left(p_{j_{k}}\right)-z_{2}^{h}\left(p_{j_{k}}\right)\right|+\left|z_{1}^{h}\left(p_{j_{0}}\right)-z_{2}^{h}\left(p_{j_{0}}\right)\right|\right] \\
& \quad \leq \varepsilon^{-1} \max _{k=1 \rightarrow d}\left[\left|z_{1}^{h}\left(p_{j_{k}}\right)-z_{2}^{h}\left(p_{j_{k}}\right)\right|+\left|z_{1}^{h}\left(p_{j_{0}}\right)-z_{2}^{h}\left(p_{j_{0}}\right)\right|\right],
\end{aligned}
$$

where we have adopted the notation of (2.6) and (2.7).

Proof. It follows immediately from (2.6), (2.7), and (2.3) that (2.28) holds. The proof of (2.29) is a straightforward adaptation of the proof of Lemma 2.1 in [7], where a similar inequality is shown for a slightly modified $F_{\varepsilon}$.

THEOREM 2.2. Let the assumptions (A) hold and $U_{\varepsilon}^{n-1} \in K^{h}, V_{\varepsilon}^{n-1} \in S^{h}$. Then for all $\varepsilon \in(0,1)$ and for all $h, \tau_{n}>0$, there exists a solution $\left\{U_{\varepsilon}^{n}, W_{\varepsilon}^{n}, V_{\varepsilon}^{n}\right\}$ to the nth step of $\left(\mathrm{P}_{\varepsilon}^{h, \tau}\right)$ with $f U_{\varepsilon}^{n}=f U_{\varepsilon}^{n-1}$ and $f V_{\varepsilon}^{n}=f V_{\varepsilon}^{n-1}$. Moreover, $U_{\varepsilon}^{n}$ is unique. In addition, $W^{n}\left(p_{j}\right)$ is unique if $\left(\pi^{h}\left[\left(U_{\varepsilon}^{n-1}\right)^{3}\right], \chi_{j}\right)>0$ for all $j \in J$.

Proof. For $n \geq 1$, given $U_{\varepsilon}^{n-1} \in K^{h}, V_{\varepsilon}^{n-1} \in S^{h}$, we define $X_{\varepsilon}^{n-1} \in Z^{h}\left(U_{\varepsilon}^{n-1}\right)$ such that

$$
\left(X_{\varepsilon}^{n-1}, \chi\right)^{h}:=\frac{1}{2}\left(\pi^{h}\left[\left(U_{\varepsilon}^{n-1}\right)^{2}\right] \nabla V_{\varepsilon}^{n-1}, \nabla \chi\right) \quad \forall \chi \in S^{h} .
$$

It follows from (2.11a), (2.27), and (2.30) that we seek $U_{\varepsilon}^{n} \in K^{h}\left(U_{\varepsilon}^{n-1}\right)$, where for all $q^{h} \in K^{h}$

$$
S^{h}\left(q^{h}\right):=\left\{\chi \in S^{h}: \chi-q^{h} \in Z^{h}\left(q^{h}\right)\right\} \quad \text { and } \quad K^{h}\left(q^{h}\right):=S^{h}\left(q^{h}\right) \cap K^{h} .
$$

In addition, we have that (cf. (2.26))

$$
W_{\varepsilon}^{n} \equiv-3 \mathcal{G}_{U_{\varepsilon}^{n-1}}^{h}\left[\frac{U_{\varepsilon}^{n}-U_{\varepsilon}^{n-1}}{\tau_{n}}+X_{\varepsilon}^{n-1}\right]+\sum_{j \in J_{0}\left(U_{\varepsilon}^{n-1}\right)} \alpha_{j}^{n} \chi_{j}+\sum_{m=1}^{M} \beta_{m}^{n} \Xi_{m}\left(U_{\varepsilon}^{n-1}\right),
$$

where $\left\{\alpha_{j}^{n}\right\}_{j \in J_{0}\left(U_{\varepsilon}^{n-1}\right)}$ and $\left\{\beta_{m}^{n}\right\}_{m=1}^{M}$ are arbitrary constants. Hence (2.11a) and (2.11b) can be restated as follows. 
For $n \geq 1$, find $U_{\varepsilon}^{n} \in K^{h}\left(U_{\varepsilon}^{n-1}\right)$ and constant Lagrange multipliers $\left\{\alpha_{j}^{n}\right\}_{j \in J_{0}\left(U_{\varepsilon}^{n-1}\right)}$, $\left\{\beta_{m}^{n}\right\}_{m=1}^{M}$ such that

$$
c\left(\nabla U_{\varepsilon}^{n}, \nabla\left(\chi-U_{\varepsilon}^{n}\right)\right)+3\left(\mathcal{G}_{U_{\varepsilon}^{n-1}}^{h}\left[\frac{U_{\varepsilon}^{n}-U_{\varepsilon}^{n-1}}{\tau_{n}}\right], \chi-U_{\varepsilon}^{n}\right)^{h}
$$

$$
\geq\left(\sum_{j \in J_{0}\left(U_{\varepsilon}^{n-1}\right)} \alpha_{j}^{n} \chi_{j}+\sum_{m=1}^{M} \beta_{m}^{n} \Xi_{m}\left(U_{\varepsilon}^{n-1}\right)+\bar{X}_{\varepsilon}^{n-1}, \chi-U_{\varepsilon}^{n}\right)^{h} \quad \forall \chi \in K^{h},
$$

where $\bar{X}_{\varepsilon}^{n-1} \in S^{h}$ is such that

$$
\left(\bar{X}_{\varepsilon}^{n-1}, \chi\right)^{h}:=-\left(\phi^{-}\left(U_{\varepsilon}^{n-1}+\varepsilon\right)+3 \mathcal{G}_{U_{\varepsilon}^{n-1}}^{h} X_{\varepsilon}^{n-1}, \chi\right)^{h} \quad \forall \chi \in S^{h} .
$$

It follows from (2.33), (2.31), and (2.24) that $U_{\varepsilon}^{n} \in K^{h}\left(U_{\varepsilon}^{n-1}\right)$ is such that

$$
A_{U_{\varepsilon}^{n-1}}\left(U_{\varepsilon}^{n}, \widetilde{z}^{h}-U_{\varepsilon}^{n}\right) \geq\left(\bar{X}_{\varepsilon}^{n-1}, \widetilde{z}^{h}-U_{\varepsilon}^{n}\right)^{h} \quad \forall \widetilde{z}^{h} \in K^{h}\left(U_{\varepsilon}^{n-1}\right),
$$

where $A_{U_{\varepsilon}^{n-1}}: S^{h}\left(U_{\varepsilon}^{n-1}\right) \times S^{h} \rightarrow \mathbb{R}$ is defined by

$$
A_{U_{\varepsilon}^{n-1}}\left(z^{h}, \chi\right):=c\left(\nabla z^{h}, \nabla \chi\right)+3\left(\mathcal{G}_{U_{\varepsilon}^{n-1}}^{h}\left[\frac{z^{h}-U_{\varepsilon}^{n-1}}{\tau_{n}}\right], \chi\right)^{h} \quad \forall z^{h} \in S^{h}\left(U_{\varepsilon}^{n-1}\right), \chi \in S^{h} .
$$

There exists $U_{\varepsilon}^{n} \in K^{h}\left(U_{\varepsilon}^{n-1}\right)$ satisfying (2.34) since, on noting (2.27), this is the Euler-Lagrange variational inequality of the convex minimization problem

$$
\min _{\widetilde{z}^{h} \in K^{h}\left(U_{\varepsilon}^{n-1}\right)}\left\{\frac{c}{2}\left|\widetilde{z}^{h}\right|_{1}^{2}+\frac{3}{2 \tau_{n}}\left|\left[\pi^{h}\left[\left(U_{\varepsilon}^{n-1}\right)^{3}\right]\right]^{\frac{1}{2}} \nabla \mathcal{G}_{U_{\varepsilon}^{n-1}}^{h}\left(\widetilde{z}^{h}-U_{\varepsilon}^{n-1}\right)\right|_{0}^{2}-\left(\bar{X}_{\varepsilon}^{n-1}, \widetilde{z}^{h}\right)^{h}\right\} .
$$

Furthermore, given any $z^{h} \in K^{h}$, similarly to $(2.26)$ there exists a $\zeta \in \mathbb{R}_{>0}$ such that

$$
\begin{aligned}
& \widetilde{z}^{h}:=U_{\varepsilon}^{n}+\zeta\left\{\left(z^{h}-U_{\varepsilon}^{n}\right)-\sum_{j \in J_{0}\left(U_{\varepsilon}^{n-1}\right)}\left(z^{h}-U_{\varepsilon}^{n}\right)\left(p_{j}\right) \chi_{j}\right. \\
&\left.-\sum_{m=1}^{M} \frac{\left(z^{h}-U_{\varepsilon}^{n}, \Xi_{m}\left(U_{\varepsilon}^{n-1}\right)\right)^{h}}{\left(U_{\varepsilon}^{n}, \Xi_{m}\left(U_{\varepsilon}^{n-1}\right)\right)^{h}} \pi^{h}\left[U_{\varepsilon}^{n} \Xi_{m}\left(U_{\varepsilon}^{n-1}\right)\right]\right\} \\
& \equiv \pi^{h}\left[\left(1-\zeta\left(1+\sum_{m=1}^{M} \frac{\left(z^{h}-U_{\varepsilon}^{n}, \Xi_{m}\left(U_{\varepsilon}^{n-1}\right)\right)^{h}}{\left(U_{\varepsilon}^{n}, \Xi_{m}\left(U_{\varepsilon}^{n-1}\right)\right)^{h}} \Xi_{m}\left(U_{\varepsilon}^{n-1}\right)\right)\right) U_{\varepsilon}^{n}\right] \\
&+\zeta\left(z^{h}-\sum_{j \in J_{0}\left(U_{\varepsilon}^{n-1}\right)} z^{h}\left(p_{j}\right) \chi_{j}\right) \in K^{h}\left(U_{\varepsilon}^{n-1}\right) .
\end{aligned}
$$

Here we have used that $\Xi_{m}\left(U_{\varepsilon}^{n-1}\right)\left(p_{j}\right)=U_{\varepsilon}^{n}\left(p_{j}\right)=0$ for all $j \in J_{0}\left(U_{\varepsilon}^{n-1}\right)$, and $\left(\pi^{h}\left[U_{\varepsilon}^{n} \Xi_{m}\left(U_{\varepsilon}^{n-1}\right)\right], \Xi_{m}\left(U_{\varepsilon}^{n-1}\right)\right)^{h}=\left(U_{\varepsilon}^{n}, \Xi_{m}\left(U_{\varepsilon}^{n-1}\right)\right)^{h}=\left(U_{\varepsilon}^{n-1}, \Xi_{m}\left(U_{\varepsilon}^{n-1}\right)\right)^{h}>0$ for 
$m=1 \rightarrow M$. For all $z^{h} \in K^{h}$, choosing $\widetilde{z}^{h} \in K^{h}\left(U_{\varepsilon}^{n-1}\right)$ (as constructed in (2.37)) in (2.34) yields the existence of a solution to (2.33) with

$$
\alpha_{j}^{n}=\frac{A_{U_{\varepsilon}^{n-1}}\left(U_{\varepsilon}^{n}, \chi_{j}\right)-\left(\bar{X}_{\varepsilon}^{n-1}, \chi_{j}\right)^{h}}{\left(1, \chi_{j}\right)} \quad \forall j \in J_{0}\left(U_{\varepsilon}^{n-1}\right)
$$

and

$$
\beta_{m}^{n}=\frac{A_{U_{\varepsilon}^{n-1}}\left(U_{\varepsilon}^{n}, \pi^{h}\left[U_{\varepsilon}^{n} \Xi_{m}\left(U_{\varepsilon}^{n-1}\right)\right]\right)-\left(\bar{X}_{\varepsilon}^{n-1}, U_{\varepsilon}^{n} \Xi_{m}\left(U_{\varepsilon}^{n-1}\right)\right)^{h}}{\left(U_{\varepsilon}^{n}, \Xi_{m}\left(U_{\varepsilon}^{n-1}\right)\right)^{h}} \quad m=1 \rightarrow M .
$$

Therefore, on noting (2.32), we have the existence of a solution $\left\{U_{\varepsilon}^{n}, W_{\varepsilon}^{n}\right\}$ to $\left(\mathrm{P}_{\varepsilon}^{h, \tau}\right)$ with $f U_{\varepsilon}^{n}=f U_{\varepsilon}^{n-1}$.

To prove the existence of $V_{\varepsilon}^{n}$, we will make use of the Brouwer fixed point theorem (see, e.g., [29, Theorem 9.36, p. 357]). Let $\mathcal{J}:=\# J$, and let $g: \mathbb{R}^{\mathcal{J}} \rightarrow \mathbb{R}^{\mathcal{J}}$ be defined by

$$
\begin{aligned}
g_{j}(\underline{V}):= & \left(V, \chi_{j}\right)^{h}+\rho \tau_{n}\left(\nabla V, \nabla \chi_{j}\right)+\tau_{n}\left(U_{\varepsilon}^{n} \Lambda_{\varepsilon}(V) \nabla V, \nabla \chi_{j}\right) \\
& +\frac{\tau_{n}}{2}\left(\pi^{h}\left[\left(U_{\varepsilon}^{n}\right)^{\frac{1}{2}}\left(U_{\varepsilon}^{n-1}\right)^{\frac{3}{2}}\right] \Lambda_{\varepsilon}(V) \nabla W_{\varepsilon}^{n}, \nabla \chi_{j}\right) \quad \forall j \in J,
\end{aligned}
$$

where $V \equiv \sum_{j \in J} V_{j} \chi_{j}$ and $\underline{V}:=\left(V_{1}, \ldots, V_{\mathcal{J}}\right)^{T} \in \mathbb{R}^{\mathcal{J}}$. Noting Lemma 2.1, we have that $g$ is continuous, and hence it is sufficient to show that $g$ is coercive. We have that

$$
\begin{aligned}
\sum_{j \in J} g_{j}(\underline{V}) V_{j} & =|V|_{h}^{2}+\rho \tau_{n}|V|_{1}^{2}+\tau_{n}\left(U_{\varepsilon}^{n} \Lambda_{\varepsilon}(V) \nabla V, \nabla V\right) \\
& +\frac{\tau_{n}}{2}\left(\pi^{h}\left[\left(U_{\varepsilon}^{n}\right)^{\frac{1}{2}}\left(U_{\varepsilon}^{n-1}\right)^{\frac{3}{2}}\right] \Lambda_{\varepsilon}(V) \nabla W_{\varepsilon}^{n}, \nabla V\right) \quad \forall V \in S^{h} .
\end{aligned}
$$

From (1.19), (2.15), and (2.28), we have

$$
\begin{gathered}
\frac{\tau_{n}}{2}\left|\left(\pi^{h}\left[\left(U_{\varepsilon}^{n}\right)^{\frac{1}{2}}\left(U_{\varepsilon}^{n-1}\right)^{\frac{3}{2}}\right] \Lambda_{\varepsilon}(V) \nabla W_{\varepsilon}^{n}, \nabla V\right)\right| \\
\quad \leq \frac{\tau_{n}}{2}\left(U_{\varepsilon}^{n} \Lambda_{\varepsilon}(V) \nabla V, \nabla V\right)+\frac{\tau_{n}}{8}\left(\pi^{h}\left[\left(U_{\varepsilon}^{n-1}\right)^{3}\right] \Lambda_{\varepsilon}(V) \nabla W_{\varepsilon}^{n}, \nabla W_{\varepsilon}^{n}\right) \\
\quad \leq \frac{\tau_{n}}{2}\left(U_{\varepsilon}^{n} \Lambda_{\varepsilon}(V) \nabla V, \nabla V\right)+C\left(\tau_{n}, U_{\varepsilon}^{n-1}, W_{\varepsilon}^{n}\right) .
\end{gathered}
$$

It follows from (2.38), (2.39), and (2.28) that

$$
\sum_{j \in J} g_{j}(\underline{V}) V_{j} \geq|V|_{h}^{2}-C\left(\tau_{n}, U_{\varepsilon}^{n-1}, W_{\varepsilon}^{n}\right) \quad \forall V \in S^{h} .
$$

Hence the coerciveness of $g$ follows from (2.40) and (2.1). Therefore, on noting the aforementioned theorem, we have the existence of $V_{\varepsilon}^{n}$ to $(2.11 \mathrm{c})$ and hence the existence of a solution $\left\{U_{\varepsilon}^{n}, W_{\varepsilon}^{n}, V_{\varepsilon}^{n}\right\}$ to $\left(\mathrm{P}_{\varepsilon}^{h, \tau}\right)$. Choosing $\chi \equiv 1$ in (2.11c) yields that $f V_{\varepsilon}^{n}=f V_{\varepsilon}^{n-1}$.

If (2.33) has two solutions $\left\{U_{\varepsilon}^{n, i},\left\{\alpha_{j}^{n, i}\right\}_{j \in J_{0}\left(U_{\varepsilon}^{n-1}\right)},\left\{\beta_{m}^{n, i}\right\}_{m=1}^{M}\right\}, i=1,2$, then it follows from (2.34) and (2.27) that $\widetilde{U}_{\varepsilon}^{n}:=U_{\varepsilon}^{n, 1}-U_{\varepsilon}^{n, 2} \in Z^{h}\left(U_{\varepsilon}^{n-1}\right)$ satisfies

$$
c\left|\widetilde{U}_{\varepsilon}^{n}\right|_{1}^{2}+3 \tau_{n}^{-1}\left|\left[\pi^{h}\left[\left(U_{\varepsilon}^{n-1}\right)^{3}\right]\right]^{\frac{1}{2}} \nabla\left(\mathcal{G}_{U_{\varepsilon}^{n-1}}^{h} \widetilde{U}_{\varepsilon}^{n}\right)\right|_{0}^{2} \leq 0 .
$$


Therefore, the uniqueness of $U_{\varepsilon}^{n}$ follows from (1.17). For any $\zeta \in(0,1)$, choosing $\chi \equiv$ $U_{\varepsilon}^{n} \pm \zeta \pi^{h}\left[U_{\varepsilon}^{n} \Xi_{m}\left(U_{\varepsilon}^{n-1}\right)\right] \equiv \pi^{h}\left[\left(1 \pm \zeta \Xi_{m}\left(U_{\varepsilon}^{n-1}\right)\right) U_{\varepsilon}^{n}\right]$ in (2.33) for $m=1 \rightarrow M$ yields the uniqueness of the Lagrange multipliers $\left\{\beta_{m}^{n}\right\}_{m=1}^{M}$. Hence the desired uniqueness result on $W_{\varepsilon}^{n}$ follows from noting (2.32).

Lemma 2.3. Let the assumptions of Theorem 2.2 hold. Then for all $\varepsilon \in(0,1)$ and for all $h, \tau_{n}>0$ a solution $\left\{U_{\varepsilon}^{n}, W_{\varepsilon}^{n}, V_{\varepsilon}^{n}\right\}$ to the nth step of $\left(\mathrm{P}_{\varepsilon}^{h, \tau}\right)$ is such that

$$
\begin{gathered}
\mathcal{E}\left(U_{\varepsilon}^{n}, V_{\varepsilon}^{n}\right)+\frac{c}{2}\left|U_{\varepsilon}^{n}-U_{\varepsilon}^{n-1}\right|_{1}^{2}+\frac{1}{2}\left|V_{\varepsilon}^{n}-V_{\varepsilon}^{n-1}\right|_{h}^{2}+\rho \tau_{n}\left(\nabla V_{\varepsilon}^{n}, \nabla \pi^{h}\left[F_{\varepsilon}^{\prime}\left(V_{\varepsilon}^{n}\right)\right]\right) \\
+\frac{\tau_{n}}{24}\left(\pi^{h}\left[\left(U_{\varepsilon}^{n-1}\right)^{3}\right] \nabla W_{\varepsilon}^{n}, \nabla W_{\varepsilon}^{n}\right)+\frac{5}{8} \tau_{n}\left(U_{\varepsilon}^{n} \nabla V_{\varepsilon}^{n}, \nabla V_{\varepsilon}^{n}\right) \\
\leq \mathcal{E}\left(U_{\varepsilon}^{n-1}, V_{\varepsilon}^{n-1}\right)+\frac{\tau_{n}}{2}\left(U_{\varepsilon}^{n-1} \nabla V_{\varepsilon}^{n-1}, \nabla V_{\varepsilon}^{n-1}\right),
\end{gathered}
$$

where

$$
\mathcal{E}\left(U_{\varepsilon}^{n}, V_{\varepsilon}^{n}\right):=\frac{c}{2}\left|U_{\varepsilon}^{n}\right|_{1}^{2}+\left(F_{\varepsilon}\left(V_{\varepsilon}^{n}\right)+\Phi^{-}\left(U_{\varepsilon}^{n}+\varepsilon\right), 1\right)^{h} .
$$

Proof. Choosing $\chi \equiv W_{\varepsilon}^{n}$ in (2.11a), $z^{h} \equiv U_{\varepsilon}^{n-1}$ in (2.11b), and $\chi \equiv \pi^{h}\left[F_{\varepsilon}^{\prime}\left(V_{\varepsilon}^{n}\right)\right]$ in $(2.11 \mathrm{c})$ and noting $(2.5)$ yield that

$$
\begin{aligned}
\left(U_{\varepsilon}^{n}-U_{\varepsilon}^{n-1}, W_{\varepsilon}^{n}\right)^{h} & +\frac{\tau_{n}}{3}\left(\pi^{h}\left[\left(U_{\varepsilon}^{n-1}\right)^{3}\right] \nabla W_{\varepsilon}^{n}, \nabla W_{\varepsilon}^{n}\right) \\
& =-\frac{\tau_{n}}{2}\left(\pi^{h}\left[\left(U_{\varepsilon}^{n-1}\right)^{2}\right] \nabla V_{\varepsilon}^{n-1}, \nabla W_{\varepsilon}^{n}\right), \\
c\left(\nabla U_{\varepsilon}^{n}, \nabla\left(U_{\varepsilon}^{n}-U_{\varepsilon}^{n-1}\right)\right) & +\left(\phi^{-}\left(U_{\varepsilon}^{n-1}+\varepsilon\right), U_{\varepsilon}^{n}-U_{\varepsilon}^{n-1}\right)^{h} \\
& \leq\left(W_{\varepsilon}^{n}, U_{\varepsilon}^{n}-U_{\varepsilon}^{n-1}\right)^{h}, \\
\left(V_{\varepsilon}^{n}-V_{\varepsilon}^{n-1}, F_{\varepsilon}^{\prime}\left(V_{\varepsilon}^{n}\right)\right)^{h} & +\rho \tau_{n}\left(\nabla V_{\varepsilon}^{n}, \nabla \pi^{h}\left[F_{\varepsilon}^{\prime}\left(V_{\varepsilon}^{n}\right)\right]\right) \\
& +\tau_{n}\left(U_{\varepsilon}^{n} \nabla V_{\varepsilon}^{n}, \nabla V_{\varepsilon}^{n}\right) \\
& =-\frac{\tau_{n}}{2}\left(\pi^{h}\left[\left(U_{\varepsilon}^{n}\right)^{\frac{1}{2}}\left(U_{\varepsilon}^{n-1}\right)^{\frac{3}{2}}\right] \nabla W_{\varepsilon}^{n}, \nabla V_{\varepsilon}^{n}\right) .
\end{aligned}
$$

On noting the elementary identity

$$
2 r(r-s)=\left(r^{2}-s^{2}\right)+(r-s)^{2} \quad \forall r, s \in \mathbb{R}
$$

and the concavity of $\Phi^{-}$, it follows from $(2.43 \mathrm{~b})$ that

$$
\begin{aligned}
\frac{c}{2}\left|U_{\varepsilon}^{n}\right|_{1}^{2}+\frac{c}{2} \mid U_{\varepsilon}^{n}- & \left.U_{\varepsilon}^{n-1}\right|_{1} ^{2}+\left(\Phi^{-}\left(U_{\varepsilon}^{n}+\varepsilon\right), 1\right)^{h} \\
& \leq \frac{c}{2}\left|U_{\varepsilon}^{n-1}\right|_{1}^{2}+\left(\Phi^{-}\left(U_{\varepsilon}^{n-1}+\varepsilon\right), 1\right)^{h}+\left(W_{\varepsilon}^{n}, U_{\varepsilon}^{n}-U_{\varepsilon}^{n-1}\right)^{h} .
\end{aligned}
$$

Combining (2.43a) and (2.45) yields that

$$
\frac{c}{2}\left|U_{\varepsilon}^{n}\right|_{1}^{2}+\left(\Phi^{-}\left(U_{\varepsilon}^{n}+\varepsilon\right), 1\right)^{h}+\frac{\tau_{n}}{3}\left(\pi^{h}\left[\left(U_{\varepsilon}^{n-1}\right)^{3}\right] \nabla W_{\varepsilon}^{n}, \nabla W_{\varepsilon}^{n}\right)+\frac{c}{2}\left|U_{\varepsilon}^{n}-U_{\varepsilon}^{n-1}\right|_{1}^{2}
$$

$$
\leq \frac{c}{2}\left|U_{\varepsilon}^{n-1}\right|_{1}^{2}+\left(\Phi^{-}\left(U_{\varepsilon}^{n-1}+\varepsilon\right), 1\right)^{h}-\frac{\tau_{n}}{2}\left(\pi^{h}\left[\left(U_{\varepsilon}^{n-1}\right)^{2}\right] \nabla V_{\varepsilon}^{n-1}, \nabla W_{\varepsilon}^{n}\right) .
$$

Now $F_{\varepsilon}^{\prime \prime} \geq 1$ implies that

$$
\left(V_{\varepsilon}^{n}-V_{\varepsilon}^{n-1}, F_{\varepsilon}^{\prime}\left(V_{\varepsilon}^{n}\right)\right)^{h} \geq\left(F_{\varepsilon}\left(V_{\varepsilon}^{n}\right)-F_{\varepsilon}\left(V_{\varepsilon}^{n-1}\right), 1\right)^{h}+\frac{1}{2}\left|V_{\varepsilon}^{n-1}-V_{\varepsilon}^{n}\right|_{h}^{2} .
$$

Combining (2.43c), (2.46), and (2.47) and noting (1.19), (2.15), and (2.42) yield that

$$
\begin{aligned}
& \mathcal{E}\left(U_{\varepsilon}^{n}, V_{\varepsilon}^{n}\right)+\frac{c}{2}\left|U_{\varepsilon}^{n}-U_{\varepsilon}^{n-1}\right|_{1}^{2}+\frac{1}{2}\left|V_{\varepsilon}^{n}-V_{\varepsilon}^{n-1}\right|_{h}^{2}+\rho \tau_{n}\left(\nabla V_{\varepsilon}^{n}, \nabla \pi^{h}\left[F_{\varepsilon}^{\prime}\left(V_{\varepsilon}^{n}\right)\right]\right)+\frac{\tau_{n}}{3}\left(\pi^{h}\left[\left(U_{\varepsilon}^{n-1}\right)^{3}\right] \nabla W_{\varepsilon}^{n}, \nabla W_{\varepsilon}^{n}\right)+\tau_{n}\left(U_{\varepsilon}^{n} \nabla V_{\varepsilon}^{n}, \nabla V_{\varepsilon}^{n}\right) \\
& \leq \mathcal{E}\left(U_{\varepsilon}^{n-1}, V_{\varepsilon}^{n-1}\right)-\frac{\tau_{n}}{2}\left(\pi^{h}\left[\left(U_{\varepsilon}^{n}\right)^{\frac{1}{2}}\left(U_{\varepsilon}^{n-1}\right)^{\frac{3}{2}}\right] \nabla W_{\varepsilon}^{n}, \nabla V_{\varepsilon}^{n}\right) \\
&-\frac{\tau_{n}}{2}\left(\pi^{h}\left[\left(U_{\varepsilon}^{n-1}\right)^{2}\right] \nabla W_{\varepsilon}^{n}, \nabla V_{\varepsilon}^{n-1}\right) \\
& \leq \mathcal{E}\left(U_{\varepsilon}^{n-1}, V_{\varepsilon}^{n-1}\right)+\frac{\zeta+\gamma}{4} \tau_{n}\left(\pi^{h}\left[\left(U_{\varepsilon}^{n-1}\right)^{3}\right] \nabla W_{\varepsilon}^{n}, \nabla W_{\varepsilon}^{n}\right) \\
&+\frac{\tau_{n}}{4 \zeta}\left(U_{\varepsilon}^{n-1} \nabla V_{\varepsilon}^{n-1}, \nabla V_{\varepsilon}^{n-1}\right)+\frac{\tau_{n}}{4 \gamma}\left(U_{\varepsilon}^{n} \nabla V_{\varepsilon}^{n}, \nabla V_{\varepsilon}^{n}\right)
\end{aligned}
$$


for arbitrary $\zeta, \gamma \in \mathbb{R}_{>0}$. Choosing $\zeta=\frac{1}{2}$ and $\gamma=\frac{2}{3}$ leads to the desired result (2.41).

THEOREM 2.4. Let the assumptions (A) hold and $U_{\varepsilon}^{0} \in K^{h}, V_{\varepsilon}^{0} \in S^{h}$. Then for all $\varepsilon \in(0,1), h>0$ a solution $\left\{U_{\varepsilon}^{n}, W_{\varepsilon}^{n}, V_{\varepsilon}^{n}\right\}_{n=1}^{N}$ to $\left(\mathrm{P}_{\varepsilon}^{h, \tau}\right)$ with $a=0\left(\phi^{-} \equiv 0\right)$ is such that $f U_{\varepsilon}^{n}=f U_{\varepsilon}^{0}$ and $f V_{\varepsilon}^{n}=f V_{\varepsilon}^{0}$, and if $\tau_{n} \leq \frac{5}{4} \omega \tau_{n-1}, n=2 \rightarrow N$, for an $\omega \in(0,1)$, then

$$
\begin{aligned}
& c \max _{1 \leq n \leq N}\left\|U_{\varepsilon}^{n}\right\|_{1}^{2}+\max _{1 \leq n \leq N}\left(F_{\varepsilon}\left(V_{\varepsilon}^{n}\right), 1\right)^{h}+\max _{1 \leq n \leq N}\left|V_{\varepsilon}^{n}\right|_{0}^{2}+\varepsilon^{-1} \max _{1 \leq n \leq N}\left|\pi^{h}\left[V_{\varepsilon}^{n}\right]_{-}\right|_{0}^{2} \\
& \quad+c \sum_{n=1}^{N}\left\|U_{\varepsilon}^{n}-U_{\varepsilon}^{n-1}\right\|_{1}^{2}+\sum_{n=1}^{N}\left|V_{\varepsilon}^{n}-V_{\varepsilon}^{n-1}\right|_{0}^{2}+\rho \sum_{n=1}^{N} \tau_{n}\left(\nabla V_{\varepsilon}^{n}, \nabla \pi^{h}\left[F_{\varepsilon}^{\prime}\left(V_{\varepsilon}^{n}\right)\right]\right) \\
& \quad+\sum_{n=1}^{N} \tau_{n}\left(\pi^{h}\left[\left(U_{\varepsilon}^{n-1}\right)^{3}\right] \nabla W_{\varepsilon}^{n}, \nabla W_{\varepsilon}^{n}\right)+(1-\omega) \sum_{n=1}^{N} \tau_{n}\left(U_{\varepsilon}^{n} \nabla V_{\varepsilon}^{n}, \nabla V_{\varepsilon}^{n}\right)
\end{aligned}
$$

$$
\begin{aligned}
& +\rho \sum_{n=1}^{N} \tau_{n}\left\|V_{\varepsilon}^{n}\right\|_{1}^{2} \leq C\left[1+\left\|U_{\varepsilon}^{0}\right\|_{1}^{2}+\left(U_{\varepsilon}^{0} \nabla V_{\varepsilon}^{0}, \nabla V_{\varepsilon}^{0}\right)+\left(F_{\varepsilon}\left(V_{\varepsilon}^{0}\right), 1\right)^{h}\right] \\
& \sum_{n=1}^{N} \tau_{n}\left|\mathcal{G}\left[\frac{U_{\varepsilon}^{n}-U_{\varepsilon}^{n-1}}{\tau_{n}}\right]\right|_{1}^{2}+\sum_{n=1}^{N} \tau_{n}\left|\mathcal{G}\left[\frac{V_{\varepsilon}^{n}-V_{\varepsilon}^{n-1}}{\tau_{n}}\right]\right|_{1}^{2}
\end{aligned}
$$

$$
\leq C\left(\max _{n=0 \rightarrow N}\left\|U_{\varepsilon}^{n}\right\|_{0, \infty}\right)\left[1+\left\|U_{\varepsilon}^{0}\right\|_{1}^{2}+\left(U_{\varepsilon}^{0} \nabla V_{\varepsilon}^{0}, \nabla V_{\varepsilon}^{0}\right)+\left(F_{\varepsilon}\left(V_{\varepsilon}^{0}\right), 1\right)^{h}\right] .
$$

Proof. Summing (2.41) from $n=1 \rightarrow k$ and observing that $\tau_{n} \leq \frac{5}{4} \omega \tau_{n-1}$, $n=2 \rightarrow k$, yield for any $k \leq N$ that

$$
\begin{gathered}
\mathcal{E}\left(U_{\varepsilon}^{k}, V_{\varepsilon}^{k}\right)+\frac{1}{2} \sum_{n=1}^{k}\left[c\left|U_{\varepsilon}^{n}-U_{\varepsilon}^{n-1}\right|_{1}^{2}+\left|V_{\varepsilon}^{n}-V_{\varepsilon}^{n-1}\right|_{h}^{2}\right]+\rho \sum_{n=1}^{k} \tau_{n}\left(\nabla V_{\varepsilon}^{n}, \nabla \pi^{h}\left[F_{\varepsilon}^{\prime}\left(V_{\varepsilon}^{n}\right)\right]\right) \\
+\frac{1}{24} \sum_{n=1}^{k} \tau_{n}\left(\pi^{h}\left[\left(U_{\varepsilon}^{n-1}\right)^{3}\right] \nabla W_{\varepsilon}^{n}, \nabla W_{\varepsilon}^{n}\right)+\frac{5}{8}(1-\omega) \sum_{n=1}^{k} \tau_{n}\left(U_{\varepsilon}^{n} \nabla V_{\varepsilon}^{n}, \nabla V_{\varepsilon}^{n}\right) \\
\leq \mathcal{E}\left(U_{\varepsilon}^{0}, V_{\varepsilon}^{0}\right)+\frac{\tau_{1}}{2}\left(U_{\varepsilon}^{0} \nabla V_{\varepsilon}^{0}, \nabla V_{\varepsilon}^{0}\right) .
\end{gathered}
$$

As $a=0$, we have that

$$
\mathcal{E}\left(U_{\varepsilon}^{n}, V_{\varepsilon}^{n}\right)=\frac{c}{2}\left|U_{\varepsilon}^{n}\right|_{1}^{2}+\left(F_{\varepsilon}\left(V_{\varepsilon}^{n}\right), 1\right)^{h} \geq 0
$$

Therefore, the bounds $1 \rightarrow 2$ and $5 \rightarrow 9$ in (2.48a) follow from (2.49), (2.50), $U_{\varepsilon}^{n}-$ $U_{\varepsilon}^{n-1} \in Z^{h}$, (1.17), (2.1), and (2.14). Combining the bound on $F_{\varepsilon}\left(V_{\varepsilon}^{n}\right)$ in (2.48a) and (2.4) yields the bounds $3 \rightarrow 4$ in (2.48a). Bounds 3 and 7 in (2.48a) yield, on noting (2.8), bound 10 in (2.48a). 
From (1.16), (2.2), (2.11a), (2.21), and (1.17), we obtain that

$$
\begin{aligned}
& \left|\mathcal{G}\left[\frac{U_{\varepsilon}^{n}-U_{\varepsilon}^{n-1}}{\tau_{n}}\right]\right|_{1}^{2}=\left(\frac{U_{\varepsilon}^{n}-U_{\varepsilon}^{n-1}}{\tau_{n}}, \mathcal{G}\left[\frac{U_{\varepsilon}^{n}-U_{\varepsilon}^{n-1}}{\tau_{n}}\right]\right)=\left(\frac{U_{\varepsilon}^{n}-U_{\varepsilon}^{n-1}}{\tau_{n}}, Q^{h} \mathcal{G}\left[\frac{U_{\varepsilon}^{n}-U_{\varepsilon}^{n-1}}{\tau_{n}}\right]\right)^{h} \\
& \quad=-\left(\frac{1}{3} \pi^{h}\left[\left(U_{\varepsilon}^{n-1}\right)^{3}\right] \nabla W_{\varepsilon}^{n}+\frac{1}{2} \pi^{h}\left[\left(U_{\varepsilon}^{n-1}\right)^{2}\right] \nabla V_{\varepsilon}^{n-1}, \nabla\left[Q^{h} \mathcal{G}\left[\frac{U_{\varepsilon}^{n}-U_{\varepsilon}^{n-1}}{\tau_{n}}\right]\right]\right) \\
& \quad \leq C\left|U_{\varepsilon}^{n-1}\right|_{0, \infty}^{\frac{3}{2}}\left[\left|\left[\pi^{h}\left[\left(U_{\varepsilon}^{n-1}\right)^{3}\right]\right]^{\frac{1}{2}} \nabla W_{\varepsilon}^{n}\right|_{0}+\left|\left(U_{\varepsilon}^{n-1}\right)^{\frac{1}{2}} \nabla V_{\varepsilon}^{n-1}\right|_{0}\right]\left|Q^{h} \mathcal{G}\left[\frac{U_{\varepsilon}^{n}-U_{\varepsilon}^{n-1}}{\tau_{n}}\right]\right|_{1} \\
& \quad \leq C\left|U_{\varepsilon}^{n-1}\right|_{0, \infty}^{3}\left[\left|\left[\pi^{h}\left[\left(U_{\varepsilon}^{n-1}\right)^{3}\right]\right]^{\frac{1}{2}} \nabla W_{\varepsilon}^{n}\right|_{0}^{2}+\left|\left(U_{\varepsilon}^{n-1}\right)^{\frac{1}{2}} \nabla V_{\varepsilon}^{n-1}\right|_{0}^{2}\right] .
\end{aligned}
$$

Similarly to (2.51), from (1.16), (2.2), (2.11c), (2.28), (2.16), (2.21), and (1.17), we obtain that

$$
\begin{aligned}
& \left|\mathcal{G}\left[\frac{V_{\varepsilon}^{n}-V_{\varepsilon}^{n-1}}{\tau_{n}}\right]\right|_{1}^{2}=\left(\frac{V_{\varepsilon}^{n}-V_{\varepsilon}^{n-1}}{\tau_{n}}, Q^{h} \mathcal{G}\left[\frac{V_{\varepsilon}^{n}-V_{\varepsilon}^{n-1}}{\tau_{n}}\right]\right)^{h}=-\rho\left(\nabla V_{\varepsilon}^{n}, \nabla\left[Q^{h} \mathcal{G}\left[\frac{V_{\varepsilon}^{n}-V_{\varepsilon}^{n-1}}{\tau_{n}}\right]\right]\right) \\
& \quad-\left(U_{\varepsilon}^{n} \Lambda_{\varepsilon}\left(V_{\varepsilon}^{n}\right) \nabla V_{\varepsilon}^{n}+\frac{1}{2} \pi^{h}\left[\left(U_{\varepsilon}^{n}\right)^{\frac{1}{2}}\left(U_{\varepsilon}^{n-1}\right)^{\frac{3}{2}}\right] \Lambda_{\varepsilon}\left(V_{\varepsilon}^{n}\right) \nabla W_{\varepsilon}^{n}, \nabla\left[Q^{h} \mathcal{G}\left[\frac{V_{\varepsilon}^{n}-V_{\varepsilon}^{n-1}}{\tau_{n}}\right]\right]\right) \\
& \quad \leq C\left[\rho^{2}\left|\nabla V_{\varepsilon}^{n}\right|_{0}^{2}+\left|U_{\varepsilon}^{n}\right|_{0, \infty}\left(\left|\left(U_{\varepsilon}^{n}\right)^{\frac{1}{2}} \nabla V_{\varepsilon}^{n}\right|_{0}^{2}+\left|\left[\pi^{h}\left[\left(U_{\varepsilon}^{n-1}\right)^{3}\right]\right]^{\frac{1}{2}} \nabla W_{\varepsilon}^{n}\right|_{0}^{2}\right)\right] .
\end{aligned}
$$

Combining (2.51), (2.52), the assumptions on $\tau_{n}$, and the bounds $8 \rightarrow 10$ in (2.48a) yields the bounds (2.48b).

Lemma 2.5. Let $u^{0}, v^{0} \in K$, and the assumptions (A) hold. On choosing $U_{\varepsilon}^{0} \equiv Q^{h} u^{0}$ and $V_{\varepsilon}^{0} \equiv Q^{h} v^{0}$, or $U_{\varepsilon}^{0} \equiv \pi^{h} u^{0}$ and $V_{\varepsilon}^{0} \equiv \pi^{h} v^{0}$ in the case $d=1$, it follows that $U_{\varepsilon}^{0}, V_{\varepsilon}^{0} \in K^{h}$ are such that for all $h>0$

$$
\left\|U_{\varepsilon}^{0}\right\|_{1}^{2}+\left(U_{\varepsilon}^{0} \nabla V_{\varepsilon}^{0}, \nabla V_{\varepsilon}^{0}\right)+\left(F_{\varepsilon}\left(V_{\varepsilon}^{0}\right), 1\right)^{h} \leq C .
$$

Proof. The desired result (2.53) follows from (2.21), (2.20), and (2.18).

2.1. Inclusion of repulsive van der Waals forces. We end this section by extending Theorems 2.2 and 2.4 and Lemmas 2.3 and 2.5 to the approximation $\left(\mathrm{P}_{\delta, \varepsilon}^{h, \tau}\right)$. In order to prove the existence of a solution to $\left(\mathrm{P}_{\delta, \varepsilon}^{h, \tau}\right)$, we need to go through a regularization procedure which is similar to that used for the logarithmic Cahn-Hilliard equation; see, e.g., [5, 3]. For this purpose we introduce, for any $\mu \in \mathbb{R}_{>0}$, the $C^{2,1}$ convex function $\Phi_{\mu}^{+}: \mathbb{R} \rightarrow \mathbb{R}_{\geq 0}$ such that

$$
\Phi_{\mu}^{+}(s):= \begin{cases}\Phi^{+}(\mu)+\phi^{+}(\mu)(s-\mu)+\frac{(s-\mu)^{2}}{2}\left(\phi^{+}\right)^{\prime}(\mu), & s \leq \mu, \\ \Phi^{+}(s), & \mu \leq s .\end{cases}
$$

We set $\phi_{\mu}^{+}(\cdot):=\left(\Phi_{\mu}^{+}\right)^{\prime}(\cdot)$ and note that $\Phi^{+}(s) \geq \Phi_{\mu}^{+}(s) \geq 0$ for all $s \in \mathbb{R}_{>0}$.

A consequence of the monotonicity of $\phi_{\mu}^{+}$and our mesh assumption (A) is that for all $\mu \in \mathbb{R}_{>0}$

$$
\left|\pi^{h}\left[\phi_{\mu}^{+}(\chi)\right]\right|_{1}^{2} \leq\left(\phi^{+}\right)^{\prime}(\mu)\left(\nabla \chi, \nabla \pi^{h}\left[\phi_{\mu}^{+}(\chi)\right]\right) \quad \forall \chi \in S^{h} ;
$$

see, for example, [15].

THEOREM 2.6. Let the assumptions (A) hold and $U_{\varepsilon}^{n-1}, V_{\varepsilon}^{n-1} \in S^{h}$ with $U_{\varepsilon}^{n-1}>$ 0 . Then for all $\varepsilon \in(0,1)$ and for all $h, \tau_{n}>0$ there exists a solution $\left\{U_{\varepsilon}^{n}, W_{\varepsilon}^{n}, V_{\varepsilon}^{n}\right\}$ to the nth step of $\left(\mathrm{P}_{\delta, \varepsilon}^{h, \tau}\right)$ with $U_{\varepsilon}^{n}>0, f U_{\varepsilon}^{n}=f U_{\varepsilon}^{n-1}$, and $f V_{\varepsilon}^{n}=f V_{\varepsilon}^{n-1}$. Moreover, $U_{\varepsilon}^{n}$ and $W_{\varepsilon}^{n}$ are unique. 
Proof. As $\mathcal{U}_{\varepsilon}^{n-1}:=\min _{x \in \bar{\Omega}} U_{\varepsilon}^{n-1}(x)>0$, we have in place of (2.32), on noting (2.12) for $\chi \equiv 1$, that

$$
W_{\varepsilon}^{n} \equiv-3 \mathcal{G}_{U_{\varepsilon}^{n-1}}^{h}\left[\frac{U_{\varepsilon}^{n}-U_{\varepsilon}^{n-1}}{\tau_{n}}+X_{\varepsilon}^{n-1}\right]+\frac{1}{\underline{m}(\Omega)}\left(\phi^{+}\left(U_{\varepsilon}^{n}\right)+\phi^{-}\left(U_{\varepsilon}^{n-1}\right), 1\right)^{h},
$$

where $X_{\varepsilon}^{n-1} \in Z^{h}$ is defined by (2.30). Hence (2.11a) and (2.12) can be restated as follows.

Find $U_{\varepsilon}^{n} \in S^{h}\left(U_{\varepsilon}^{n-1}\right)$ such that

$$
A_{U_{\varepsilon}^{n-1}}\left(U_{\varepsilon}^{n}, \chi\right)+\left(\phi^{+}\left(U_{\varepsilon}^{n}\right),(I-f) \chi\right)^{h}=\left(\bar{X}_{\delta, \varepsilon}^{n-1}, \chi\right)^{h} \quad \forall \chi \in S^{h},
$$

where $A_{U_{\varepsilon}^{n-1}}(\cdot, \cdot)$ is defined as in (2.35) and $\bar{X}_{\delta, \varepsilon}^{n-1} \in Z^{h}$ is such that

$$
\left(\bar{X}_{\delta, \varepsilon}^{n-1}, \chi\right)^{h}:=-\left(\phi^{-}\left(U_{\varepsilon}^{n-1}\right)+3 \mathcal{G}_{U_{\varepsilon}^{n-1}}^{h} X_{\varepsilon}^{n-1},(I-f) \chi\right)^{h} \quad \forall \chi \in S^{h} .
$$

Due to the singular nature of the nonlinearity $\phi^{+}(s)$, we have to go through a regularization procedure in order to prove the existence of a solution to (2.57). For any $\mu \in \mathbb{R}_{>0}$, we introduce the regularized version of (2.57): Find $U_{\varepsilon, \mu}^{n} \in S^{h}\left(U_{\varepsilon}^{n-1}\right)$ such that

$$
A_{U_{\varepsilon}^{n-1}}\left(U_{\varepsilon, \mu}^{n}, \chi\right)+\left(\phi_{\mu}^{+}\left(U_{\varepsilon, \mu}^{n}\right),(I-f) \chi\right)^{h}=\left(\bar{X}_{\delta, \varepsilon}^{n-1}, \chi\right)^{h} \quad \forall \chi \in S^{h},
$$

where $\phi_{\mu}^{+}$is defined via (2.54). Similarly to (2.36), there exists a unique $U_{\varepsilon, \mu}^{n}$ satisfying (2.58) since this is the Euler-Lagrange equation of the convex minimization problem

$$
\min \left\{\frac{c}{2}|\chi|_{1}^{2}+\left(\Phi_{\mu}^{+}(\chi), 1\right)^{h}+\frac{3}{2 \tau_{n}}\left|\left[\pi^{h}\left[\left(U_{\varepsilon}^{n-1}\right)^{3}\right]\right]^{\frac{1}{2}} \nabla \mathcal{G}_{U_{\varepsilon}^{n-1}}^{h}\left(\chi-U_{\varepsilon}^{n-1}\right)\right|_{0}^{2}-\left(\bar{X}_{\delta, \varepsilon}^{n-1}, \chi\right)^{h}\right\} .
$$
$\chi \in S^{h}\left(U_{\varepsilon}^{n-1}\right)$

Choosing $\chi \equiv U_{\varepsilon, \mu}^{n}-U_{\varepsilon}^{n-1} \in Z^{h}$ in (2.58) and rearranging using (2.44), (2.27), (1.17), and the convexity of $\Phi_{\mu}^{+} \leq \Phi^{+}$yield that

$$
\begin{aligned}
c\left\|U_{\varepsilon, \mu}^{n}\right\|_{1}^{2}+\tau_{n}\left|\left[\pi^{h}\left[\left(U_{\varepsilon}^{n-1}\right)^{3}\right]\right]^{\frac{1}{2}} \nabla \mathcal{G}_{U_{\varepsilon}^{n-1}}^{h}\left[\frac{U_{\varepsilon, \mu}^{n}-U_{\varepsilon}^{n-1}}{\tau_{n}}\right]\right|_{0}^{2} \\
\leq C\left[\left(\Phi^{+}\left(U_{\varepsilon}^{n-1}\right), 1\right)^{h}+\left|\bar{X}_{\delta, \varepsilon}^{n-1}\right|_{h}^{2}+\left\|U_{\varepsilon}^{n-1}\right\|_{1}^{2}\right] \leq C,
\end{aligned}
$$

where, in the above and below, $C \in \mathbb{R}_{>0}$ is also independent of $\mu$. Choosing $\chi \equiv$ $\pi^{h}\left[\phi_{\mu}^{+}\left(U_{\varepsilon, \mu}^{n}\right)\right]$ in (2.58) and noting (2.55), $\bar{X}_{\delta, \varepsilon}^{n-1} \in Z^{h},(2.27),(2.14)$, (1.17), and (2.59) yield that

$\tau_{n}\left|(I-f) \pi^{h}\left[\phi_{\mu}^{+}\left(U_{\varepsilon, \mu}^{n}\right)\right]\right|_{h}^{2} \leq C \tau_{n}\left[\left|\bar{X}_{\delta, \varepsilon}^{n-1}\right|_{h}^{2}+\left|\mathcal{G}_{U_{\varepsilon}^{n-1}}^{h}\left[\frac{U_{\varepsilon, \mu}^{n}-U_{\varepsilon}^{n-1}}{\tau_{n}}\right]\right|_{h}^{2}\right]$

$$
\leq C\left(\mathcal{U}_{\varepsilon}^{n-1}\right) \tau_{n}\left[\left|\bar{X}_{\delta, \varepsilon}^{n-1}\right|_{h}^{2}+\left|\left[\pi^{h}\left[\left(U_{\varepsilon}^{n-1}\right)^{3}\right]\right]^{\frac{1}{2}} \nabla \mathcal{G}_{U_{\varepsilon}^{n-1}}^{h}\left[\frac{U_{\varepsilon, \mu}^{n}-U_{\varepsilon}^{n-1}}{\tau_{n}}\right]\right|_{0}^{2}\right] \leq C\left(\mathcal{U}_{\varepsilon}^{n-1}\right) .
$$

Choosing $\chi \equiv U_{\varepsilon, \mu}^{n}$ in (2.58) and noting the convexity of $\Phi_{\mu}^{+}$, it follows for any constant $\zeta \in \mathbb{R}_{>0}$ that

$$
\begin{aligned}
& \left(\phi_{\mu}^{+}\left(U_{\varepsilon, \mu}^{n}\right), \zeta-f U_{\varepsilon, \mu}^{n}\right)^{h} \\
& \quad \leq\left(\Phi_{\mu}^{+}(\zeta)-\Phi_{\mu}^{+}\left(U_{\varepsilon, \mu}^{n}\right), 1\right)^{h}+\left(\bar{X}_{\delta, \varepsilon}^{n-1}-3 \mathcal{G}_{U_{\varepsilon}^{n-1}}^{h}\left[\frac{U_{\varepsilon, \mu}^{n}-U_{\varepsilon}^{n-1}}{\tau_{n}}\right], U_{\varepsilon, \mu}^{n}\right)^{h} \\
& \quad \leq\left(\Phi^{+}(\zeta), 1\right)^{h}+\left(\bar{X}_{\delta, \varepsilon}^{n-1}-3 \mathcal{G}_{U_{\varepsilon}^{n-1}}^{h}\left[\frac{U_{\varepsilon, \mu}^{n}-U_{\varepsilon}^{n-1}}{\tau_{n}}\right], U_{\varepsilon, \mu}^{n}\right)^{h} .
\end{aligned}
$$


Choosing $\zeta=\left(f U_{\varepsilon, \mu}^{n}\right) \pm \frac{1}{2} \mathcal{U}_{\varepsilon}^{n-1}=\left(f U_{\varepsilon}^{n-1}\right) \pm \frac{1}{2} \mathcal{U}_{\varepsilon}^{n-1} \geq \frac{1}{2} \mathcal{U}_{\varepsilon}^{n-1}>0$ in (2.61) and noting (2.59) and (2.60) yield that

$$
\tau_{n}\left|f\left(\pi^{h}\left[\phi_{\mu}^{+}\left(U_{\varepsilon, \mu}^{n}\right)\right]\right)\right|_{h}^{2} \leq C\left(\mathcal{U}_{\varepsilon}^{n-1}\right) .
$$

It follows from (2.59), (2.60), and (2.62) that there exist $U_{\varepsilon}^{n} \in S^{h}\left(U_{\varepsilon}^{n-1}\right), \phi_{h}^{+} \in$ $S^{h}$, and a subsequence $\left\{U_{\varepsilon, \mu^{\prime}}^{n}, \pi^{h}\left[\phi_{\mu^{\prime}}^{+}\left(U_{\varepsilon, \mu^{\prime}}^{n}\right)\right]\right\}_{\mu^{\prime}}$ such that $U_{\varepsilon, \mu^{\prime}}^{n} \rightarrow U_{\varepsilon}^{n}$ and $\pi^{h}\left[\phi_{\mu^{\prime}}^{+}\left(U_{\varepsilon, \mu^{\prime}}^{n}\right)\right] \rightarrow \phi_{h}^{+}$as $\mu^{\prime} \rightarrow 0$. Noting that for all $s \in \mathbb{R},\left[\phi_{\mu}^{+}\right]^{-1}(s) \rightarrow\left[\phi^{+}\right]^{-1}(s)$ as $\mu \rightarrow 0$, we have that $\left(U_{\varepsilon}^{n}\left(p_{j}\right)-\left[\phi^{+}\right]^{-1}(s)\right)\left(\phi_{h}^{+}\left(p_{j}\right)-s\right) \geq 0$ for all $s \in \mathbb{R}, j \in J$ and hence that $\phi_{h}^{+} \equiv \pi^{h}\left[\phi^{+}\left(U_{\varepsilon}^{n}\right)\right]$. Therefore, we may pass to the limit $\mu^{\prime} \rightarrow 0$ in (2.58) to prove the existence of a solution $U_{\varepsilon}^{n}>0$ to (2.57). Uniqueness of this solution follows from the monotonicity of $\phi^{+}$. Hence noting (2.56), we have existence and uniqueness of a solution $\left\{U_{\varepsilon}^{n}, W_{\varepsilon}^{n}\right\}$ to (2.11a) and (2.12). Finally, existence of a solution $V_{\varepsilon}^{n}$ to (2.11c) follows as in the proof of Theorem 2.2 .

LEmma 2.7. Let the assumptions of Theorem 2.6 hold. Then for all $\varepsilon \in(0,1)$ and for all $h, \tau_{n}>0$ a solution $\left\{U_{\varepsilon}^{n}, W_{\varepsilon}^{n}, V_{\varepsilon}^{n}\right\}$ to the nth step of $\left(\mathrm{P}_{\delta, \varepsilon}^{h, \tau}\right)$ is such that (2.41) holds with $\mathcal{E}(\cdot, \cdot)$ replaced by $\mathcal{E}_{\delta}\left(U_{\varepsilon}^{n}, V_{\varepsilon}^{n}\right):=\frac{c}{2}\left|U_{\varepsilon}^{n}\right|_{1}^{2}+\left(F_{\varepsilon}\left(V_{\varepsilon}^{n}\right)+\Phi\left(U_{\varepsilon}^{n}\right), 1\right)^{h}$.

Proof. The proof is a straightforward adaptation of the proof of Lemma 2.3 on noting (2.9) and the convexity of $\Phi^{+}$.

THEOREM 2.8. Let the assumptions (A) hold and $U_{\varepsilon}^{0}, V_{\varepsilon}^{0} \in S^{h}$ with $U_{\varepsilon}^{0}>0$. Then for all $\varepsilon \in(0,1), h>0$, a solution $\left\{U_{\varepsilon}^{n}, W_{\varepsilon}^{n}, V_{\varepsilon}^{n}\right\}_{n=1}^{N}$ to $\left(\mathrm{P}_{\delta, \varepsilon}^{h, \tau}\right)$ is such that $f U_{\varepsilon}^{n}=f U_{\varepsilon}^{0}$ and $f V_{\varepsilon}^{n}=f V_{\varepsilon}^{0}$, and if $\tau_{n} \leq \frac{5}{4} \omega \tau_{n-1}, n=2 \rightarrow N$, for an $\omega \in(0,1)$, then $(2.48 \mathrm{a}, \mathrm{b})$ hold with the additional terms $\max _{1 \leq n \leq N}\left(\Phi\left(U_{\varepsilon}^{n}\right), 1\right)^{h}$ on the left-hand side of $(2.48 \mathrm{a})$ and $\left(\Phi\left(U_{\varepsilon}^{0}\right), 1\right)^{h}$ inside the square brackets on the right-hand sides of $(2.48 \mathrm{a}, \mathrm{b})$.

Proof. A straightforward adaptation of the proof of Theorem 2.4 on noting (2.10) yields the desired result.

LEMmA 2.9. Let $u^{0}, v^{0} \in K$, with $u^{0} \in L^{\infty}(\Omega)$ and $u^{0}(x) \geq \zeta>0$ for a.e. $x \in \Omega$, and let the assumptions $(\mathrm{A})$ hold. On choosing $U_{\varepsilon}^{0} \equiv Q^{h} u^{0}$ and $V_{\varepsilon}^{0} \equiv Q^{h} v^{0}$, or $U_{\varepsilon}^{0} \equiv \pi^{h} u^{0}$ and $V_{\varepsilon}^{0} \equiv \pi^{h} v^{0}$ in the case $d=1$, it follows that $U_{\varepsilon}^{0}, V_{\varepsilon}^{0} \in K^{h}$ with $U_{\varepsilon}^{0} \geq \zeta$ are such that for all $h>0$

$$
\left\|U_{\varepsilon}^{0}\right\|_{1}^{2}+\left(U_{\varepsilon}^{0} \nabla V_{\varepsilon}^{0}, \nabla V_{\varepsilon}^{0}\right)+\left(F_{\varepsilon}\left(V_{\varepsilon}^{0}\right)+\Phi\left(U_{\varepsilon}^{0}\right), 1\right)^{h} \leq C .
$$

Proof. The desired result (2.63) follows from (2.21), (2.20), and (2.18).

Remark 2.10. We note that Lemmas 2.3 and 2.7 are the discrete analogues of the energy estimates (1.5) and (1.11), respectively, on recalling (1.6), (1.7), and that $\sigma(s):=1-s$.

\section{Convergence in one space dimension. Let}

$$
\begin{aligned}
& U_{\varepsilon}(t):=\frac{t-t_{n-1}}{\tau_{n}} U_{\varepsilon}^{n}+\frac{t_{n}-t}{\tau_{n}} U_{\varepsilon}^{n-1}, \quad t \in\left[t_{n-1}, t_{n}\right], \quad n \geq 1, \\
& U_{\varepsilon}^{+}(t):=U_{\varepsilon}^{n}, \quad U_{\varepsilon}^{-}(t):=U_{\varepsilon}^{n-1}, \quad t \in\left(t_{n-1}, t_{n}\right], \quad n \geq 1 \text {. }
\end{aligned}
$$

We note for future reference that

$$
U_{\varepsilon}-U_{\varepsilon}^{ \pm}=\left(t-t_{n}^{ \pm}\right) \frac{\partial U_{\varepsilon}}{\partial t}, \quad t \in\left(t_{n-1}, t_{n}\right) \quad n \geq 1
$$

where $t_{n}^{+}:=t_{n}$ and $t_{n}^{-}:=t_{n-1}$. We introduce also $\bar{\tau}(t):=\tau_{n}$ for $t \in\left(t_{n-1}, t_{n}\right]$ and $n \geq 1$. Using the above notation and introducing analogous notation for $W_{\varepsilon}$ and $V_{\varepsilon}$, $(2.11 \mathrm{a}-\mathrm{c})$ can be restated as follows. 
Find $\left\{U_{\varepsilon}, W_{\varepsilon}, V_{\varepsilon}\right\} \in H^{1}\left(0, T ; S^{h}\right) \times L^{2}\left(0, T ; S^{h}\right) \times H^{1}\left(0, T ; S^{h}\right)$ such that $U_{\varepsilon}(\cdot, t) \in$ $K^{h}$ and for all $\chi \in L^{2}\left(0, T ; S^{h}\right), z^{h} \in L^{2}\left(0, T ; K^{h}\right)$,

$$
\int_{0}^{T}\left[\left(\frac{\partial U_{\varepsilon}}{\partial t}, \chi\right)^{h}+\frac{1}{3}\left(\pi^{h}\left[\left(U_{\varepsilon}^{-}\right)^{3}\right] \nabla W_{\varepsilon}^{+}, \nabla \chi\right)\right] \mathrm{d} t=-\frac{1}{2} \int_{0}^{T}\left(\pi^{h}\left[\left(U_{\varepsilon}^{-}\right)^{2}\right] \nabla V_{\varepsilon}^{-}, \nabla \chi\right) \mathrm{d} t,
$$

$$
\begin{aligned}
& \int_{0}^{T}\left[c\left(\nabla U_{\varepsilon}^{+}, \nabla\left(z^{h}-U_{\varepsilon}^{+}\right)\right)+\left(\phi^{-}\left(U_{\varepsilon}^{-}+\varepsilon\right)-W_{\varepsilon}^{+}, z^{h}-U_{\varepsilon}^{+}\right)^{h}\right] \mathrm{d} t \geq 0, \\
& \int_{0}^{T}\left[\left(\frac{\partial V_{\varepsilon}}{\partial t}, \chi\right)^{h}+\rho\left(\nabla V_{\varepsilon}^{+}, \nabla \chi\right)+\left(U_{\varepsilon}^{+} \Lambda_{\varepsilon}\left(V_{\varepsilon}^{+}\right) \nabla V_{\varepsilon}^{+}, \nabla \chi\right)\right] \mathrm{d} t
\end{aligned}
$$

$$
=-\frac{1}{2} \int_{0}^{T}\left(\pi^{h}\left[\left(U_{\varepsilon}^{+}\right)^{\frac{1}{2}}\left(U_{\varepsilon}^{-}\right)^{\frac{3}{2}}\right] \Lambda_{\varepsilon}\left(V_{\varepsilon}^{+}\right) \nabla W_{\varepsilon}^{+}, \nabla \chi\right) \mathrm{d} t
$$

Lemma 3.1. Let $d=1, a=0\left(\phi^{-} \equiv 0\right), \rho>0$, and $u^{0}, v^{0} \in K$ with $u^{0} \not \equiv 0$. Let $\left\{\mathcal{T}^{h}, U_{\varepsilon}^{0}, V_{\varepsilon}^{0},\left\{\tau_{n}\right\}_{n=1}^{N}, \varepsilon\right\}_{h>0}$ be such that

(i) $U_{\varepsilon}^{0} \equiv \pi^{h} u^{0}, V_{\varepsilon}^{0} \equiv \pi^{h} v^{0}$;

(ii) $\Omega$ and $\left\{\mathcal{T}^{h}\right\}_{h>0}$ fulfil assumption (A), $\varepsilon \in(0,1)$, and $\tau_{n} \leq \frac{5}{4} \omega \tau_{n-1}, n=$ $2 \rightarrow N$, for an $\omega \in(0,1)$;

(iii) $\varepsilon, \tau \rightarrow 0$ as $h \rightarrow 0$.

Then there exist a subsequence of $\left\{U_{\varepsilon}, V_{\varepsilon}\right\}_{h}$, where $\left\{U_{\varepsilon}, W_{\varepsilon}, V_{\varepsilon}\right\}$ solve $\left(\mathrm{P}_{\varepsilon}^{h, \tau}\right)$, and functions

$$
\begin{aligned}
& u \in L^{\infty}(0, T ; K) \cap H^{1}\left(0, T ;\left(H^{1}(\Omega)\right)^{\prime}\right) \cap C_{x, t}^{\frac{1}{2}, \frac{1}{8}}\left(\bar{\Omega}_{T}\right), \\
& v \in L^{\infty}\left(0, T ; L^{2}(\Omega)\right) \cap L^{2}(0, T ; K) \cap H^{1}\left(0, T ;\left(H^{1}(\Omega)\right)^{\prime}\right)
\end{aligned}
$$

with $u(x, 0)=u^{0}(x)$ for all $x \in \bar{\Omega}, v(\cdot, 0)=v^{0}(\cdot)$ in $\left(H^{1}(\Omega)\right)^{\prime}, f u(\cdot, t)=f u^{0}>0$ for all $t \in[0, T]$ and $f v(\cdot, t)=f v^{0}$ for a.e. $t \in[0, T]$ such that as $h \rightarrow 0$

$$
\begin{array}{llll}
U_{\varepsilon}, U_{\varepsilon}^{ \pm} \rightarrow u & & & \text { uniformly on } \bar{\Omega}_{T} \\
U_{\varepsilon}, U_{\varepsilon}^{ \pm} \rightarrow u & \text { and } & \mathcal{G} \frac{\partial U_{\varepsilon}}{\partial t} \rightarrow \mathcal{G} \frac{\partial u}{\partial t} & \text { weakly in } L^{2}\left(0, T ; H^{1}(\Omega)\right), \\
V_{\varepsilon}, V_{\varepsilon}^{ \pm} \rightarrow v & \text { and } & \mathcal{G} \frac{\partial V_{\varepsilon}}{\partial t} \rightarrow \mathcal{G} \frac{\partial v}{\partial t} & \text { weakly in } L^{2}\left(0, T ; H^{1}(\Omega)\right), \\
V_{\varepsilon}, V_{\varepsilon}^{ \pm} \rightarrow v & \text { and } & \Lambda_{\varepsilon}\left(V_{\varepsilon}^{+}\right) \rightarrow \lambda(v) & \text { strongly in } L^{2}\left(\Omega_{T}\right) .
\end{array}
$$

Proof. From (2.48a), (2.53), and (1.15), we have for $d=1$

$$
\max _{1 \leq n \leq N}\left\|U_{\varepsilon}^{n}\right\|_{1} \leq C \quad \Longrightarrow \quad \max _{1 \leq n \leq N}\left|U_{\varepsilon}^{n}\right|_{0, \infty} \leq C .
$$

Noting the definitions $(3.1 \mathrm{a}, \mathrm{b})$ and $(3.7)$, the bounds in $(2.48 \mathrm{a}, \mathrm{b})$ together with (1.17), (2.53), and the time step control in (ii) imply that

$$
\begin{aligned}
& \left\|U_{\varepsilon}\right\|_{L^{\infty}\left(0, T ; H^{1}(\Omega)\right)}^{2}+\left\|V_{\varepsilon}\right\|_{L^{\infty}\left(0, T ; L^{2}(\Omega)\right)}^{2}+\rho\left\|V_{\varepsilon}\right\|_{L^{2}\left(0, T ; H^{1}(\Omega)\right)}^{2}+\rho\left\|V_{\varepsilon}^{ \pm}\right\|_{L^{2}\left(0, T ; H^{1}(\Omega)\right)}^{2} \\
& \quad+\varepsilon^{-1}\left\|\pi^{h}\left[V_{\varepsilon}^{+}\right]_{-}\right\|_{L^{\infty}\left(0, T ; L^{2}(\Omega)\right)}^{2}+\left\|\bar{\tau}^{\frac{1}{2}} \frac{\partial U_{\varepsilon}}{\partial t}\right\|_{L^{2}\left(0, T ; H^{1}(\Omega)\right)}^{2}+\left\|\bar{\tau}^{\frac{1}{2}} \frac{\partial V_{\varepsilon}}{\partial t}\right\|_{L^{2}\left(\Omega_{T}\right)}^{2} \\
& \quad+\left\|\left[\pi^{h}\left[\left(U_{\varepsilon}^{-}\right)^{3}\right]\right]^{\frac{1}{2}} \nabla W_{\varepsilon}^{+}\right\|_{L^{2}\left(\Omega_{T}\right)}^{2}+\left\|\mathcal{G} \frac{\partial U_{\varepsilon}}{\partial t}\right\|_{L^{2}\left(0, T ; H^{1}(\Omega)\right)}^{2}+\left\|\mathcal{G} \frac{\partial V_{\varepsilon}}{\partial t}\right\|_{L^{2}\left(0, T ; H^{1}(\Omega)\right)}^{2} \leq C .
\end{aligned}
$$


In addition, we deduce from (3.2) and (3.8) that

$$
\begin{aligned}
\left\|U_{\varepsilon}-U_{\varepsilon}^{ \pm}\right\|_{L^{2}\left(0, T ; H^{1}(\Omega)\right)}^{2}+\left\|V_{\varepsilon}-V_{\varepsilon}^{ \pm}\right\|_{L^{2}\left(\Omega_{T}\right)}^{2} & \leq\left\|\bar{\tau} \frac{\partial U_{\varepsilon}}{\partial t}\right\|_{L^{2}\left(0, T ; H^{1}(\Omega)\right)}^{2}+\left\|\bar{\tau} \frac{\partial V_{\varepsilon}}{\partial t}\right\|_{L^{2}\left(\Omega_{T}\right)}^{2} \\
& \leq C \tau .
\end{aligned}
$$

Moreover, the first and ninth bound in (3.8) imply that the $C_{x, t}^{\frac{1}{2}, \frac{1}{8}}\left(\bar{\Omega}_{T}\right)$ norm of $U_{\varepsilon}$ is bounded independently of $h, \tau, \varepsilon$, and $T$; see, e.g., [4, Theorem 2.2]. Therefore, by the Arzelà-Ascoli theorem there exists a subsequence $\left\{U_{\varepsilon}, V_{\varepsilon}\right\}_{h}$ and a $u \geq 0$, as $U_{\varepsilon}(\cdot, t) \in K^{h}$, such that

$$
U_{\varepsilon}, U_{\varepsilon}^{ \pm} \rightarrow u \in C_{x, t}^{\frac{1}{2}, \frac{1}{8}}\left(\bar{\Omega}_{T}\right) \quad \text { uniformly on } \bar{\Omega}_{T} \text { as } h \rightarrow 0 .
$$

Furthermore, (3.10), (3.8), (2.19), (3.1a,b), (3.9), $f U_{\varepsilon}^{n}=f U_{\varepsilon}^{0}, f V_{\varepsilon}^{n}=f V_{\varepsilon}^{0}$, and our assumptions (iii) imply, as $\rho>0$, that this same subsequence $\left\{U_{\varepsilon}, V_{\varepsilon}\right\}_{h}$ can be chosen such that (3.4a), (3.4b) with $K$ replaced by $H^{1}(\Omega),(3.5 \mathrm{a}, \mathrm{b})$, and (3.6a) hold. The strong convergence result for $V_{\varepsilon}^{( \pm)}$in $(3.6 \mathrm{~b})$ follows immediately from (3.6a) and a standard embedding result. Furthermore, it follows from (3.8), (3.6a), (1.18), and a standard compactness argument that

$$
V_{\varepsilon} \rightarrow v \quad \text { in } \quad C\left([0, T] ;\left(H^{1}(\Omega)\right)^{\prime}\right) .
$$

Noting the assumptions (i) and (2.19), we have that

$$
U_{\varepsilon}^{0} \rightarrow u^{0} \quad \text { and } \quad V_{\varepsilon}^{0} \rightarrow v^{0} \quad \text { strongly in } \quad H^{1}(\Omega) .
$$

Combining (3.12), (3.11), and (3.10) yields that $u(\cdot, 0)=u^{0}(\cdot)$ in $C(\bar{\Omega})$ and $v(\cdot, 0)=$ $v^{0}(\cdot)$ in $\left(H^{1}(\Omega)\right)^{\prime}$.

We now prove the remaining result in (3.6b). For this we introduce for all $\varepsilon \in$ $(0,1), \lambda_{\varepsilon}: \mathbb{R} \rightarrow[\varepsilon, 1]$ defined, on recalling (1.13), by

$$
\lambda_{\varepsilon}(s):=[\lambda(s)-\varepsilon]_{+}+\varepsilon, \quad \text { where } \quad[s]_{+}:=\max \{s, 0\} .
$$

Then we have that

$$
\begin{aligned}
& \left\|\lambda(v)-\Lambda_{\varepsilon}\left(V_{\varepsilon}^{+}\right)\right\|_{L^{2}\left(\Omega_{T}\right)} \leq\left\|\lambda(v)-\lambda\left(V_{\varepsilon}^{+}\right)\right\|_{L^{2}\left(\Omega_{T}\right)}+\left\|\left(I-\pi^{h}\right) \lambda\left(V_{\varepsilon}^{+}\right)\right\|_{L^{2}\left(\Omega_{T}\right)} \\
& \quad+\left\|\pi^{h}\left[\lambda\left(V_{\varepsilon}^{+}\right)-\lambda_{\varepsilon}\left(V_{\varepsilon}^{+}\right)\right]\right\|_{L^{2}\left(\Omega_{T}\right)}+\left\|\pi^{h}\left[\lambda_{\varepsilon}\left(V_{\varepsilon}^{+}\right)\right]-\Lambda_{\varepsilon}\left(V_{\varepsilon}^{+}\right)\right\|_{L^{2}\left(\Omega_{T}\right)} .
\end{aligned}
$$

Noting the global Lipschitz continuity of $\lambda$, (2.18), and (3.8), we have that

$$
\begin{aligned}
\| \lambda(v) & -\lambda\left(V_{\varepsilon}^{+}\right)\left\|_{L^{2}\left(\Omega_{T}\right)}+\right\|\left(I-\pi^{h}\right) \lambda\left(V_{\varepsilon}^{+}\right) \|_{L^{2}\left(\Omega_{T}\right)} \\
& \leq\left\|v-V_{\varepsilon}^{+}\right\|_{L^{2}\left(\Omega_{T}\right)}+C h\left\|\nabla\left[\lambda\left(V_{\varepsilon}^{+}\right)\right]\right\|_{L^{2}\left(\Omega_{T}\right)} \\
& \leq\left\|v-V_{\varepsilon}^{+}\right\|_{L^{2}\left(\Omega_{T}\right)}+C h\left\|\nabla V_{\varepsilon}^{+}\right\|_{L^{2}\left(\Omega_{T}\right)} \leq\left\|v-V_{\varepsilon}^{+}\right\|_{L^{2}\left(\Omega_{T}\right)}+C h .
\end{aligned}
$$

It follows from (2.1), (2.14), (3.1b), (1.13), (3.13), and (3.8) that

$$
\begin{aligned}
& \left\|\pi^{h}\left[\lambda\left(V_{\varepsilon}^{+}\right)-\lambda_{\varepsilon}\left(V_{\varepsilon}^{+}\right)\right]\right\|_{L^{2}\left(\Omega_{T}\right)}^{2} \leq \sum_{n=1}^{N} \tau_{n}\left|\pi^{h}\left[\lambda\left(V_{\varepsilon}^{n}\right)-\lambda_{\varepsilon}\left(V_{\varepsilon}^{n}\right)\right]\right|_{h}^{2} \\
& \leq \sum_{n=1}^{N} \tau_{n}\left|\pi^{h}\left[\varepsilon-\left[V_{\varepsilon}^{n}\right]_{-}\right]\right|_{h}^{2} \leq C\left[\varepsilon^{2}+\sum_{n=1}^{N} \tau_{n}\left|\pi^{h}\left[V_{\varepsilon}^{n}\right]_{-}\right|_{0}^{2}\right] \leq C \varepsilon^{2} .
\end{aligned}
$$


From (2.7), (2.3), and (3.13), we have that $\left.\Lambda_{\varepsilon}\left(V_{\varepsilon}^{n}\right)\right|_{\left(p_{j-1}, p_{j}\right)}$ lies between $\lambda_{\varepsilon}\left(V_{\varepsilon}^{n}\left(p_{j-1}\right)\right)$ and $\lambda_{\varepsilon}\left(V_{\varepsilon}^{n}\left(p_{j}\right)\right)$ for $j=1 \rightarrow J$ and $n=1 \rightarrow N$. This together with (2.18), the global Lipschitz continuity of $\lambda_{\varepsilon}$, and (3.8) implies that

$$
\begin{aligned}
\| \pi^{h}\left[\lambda_{\varepsilon}\left(V_{\varepsilon}^{+}\right)\right]-\Lambda_{\varepsilon}\left(V_{\varepsilon}^{+}\right) & \left\|_{L^{2}\left(\Omega_{T}\right)} \leq C h\right\| \nabla \pi^{h}\left[\lambda_{\varepsilon}\left(V_{\varepsilon}^{+}\right)\right] \|_{L^{2}\left(\Omega_{T}\right)} \\
& \leq C h\left\|\nabla\left[\lambda_{\varepsilon}\left(V_{\varepsilon}^{+}\right)\right]\right\|_{L^{2}\left(\Omega_{T}\right)} \leq C h\left\|\nabla V_{\varepsilon}^{+}\right\|_{L^{2}\left(\Omega_{T}\right)} \leq C h .
\end{aligned}
$$

Combining (3.14), (3.15), (3.16), and (3.17) and noting the result on $V_{\varepsilon}^{+}$in (3.6b) and our assumption (iii) on $\varepsilon$ yield the desired result on $\Lambda_{\varepsilon}\left(V_{\varepsilon}^{+}\right)$in (3.6b). Finally, we note that $\Lambda_{\varepsilon}\left(V_{\varepsilon}^{+}\right) \geq 0$ and $(3.6 \mathrm{~b}) \Rightarrow \lambda(v) \geq 0$ a.e. $\Rightarrow v \geq 0$ a.e. $\Rightarrow K$ in (3.4b).

For any $\alpha>0$, we set

$$
B_{\alpha}:=\left\{(x, t) \in \bar{\Omega}_{T}: u(x, t)>\alpha\right\} \quad \text { and } \quad B_{\alpha}(t):=\{x \in \bar{\Omega}: u(x, t)>\alpha\} .
$$

From (3.4a), we have that there exist positive constants $C_{x}$ and $C_{t}$ such that

$$
\begin{array}{lll}
\left|u\left(y_{2}, t\right)-u\left(y_{1}, t\right)\right| \leq C_{x}\left|y_{2}-y_{1}\right|^{\frac{1}{2}} & \forall y_{1}, y_{2} \in \bar{\Omega}, \quad \forall t \in[0, T] ; \\
\left|u\left(x, t_{b}\right)-u\left(x, t_{a}\right)\right| \leq C_{t}\left|t_{b}-t_{a}\right|^{\frac{1}{8}} & \forall t_{a}, t_{b} \in[0, T], \quad \forall x \in \bar{\Omega} .
\end{array}
$$

As $f u(\cdot, t)=f u^{0}>0$ for all $t \in[0, T]$, it follows that there exists an $\alpha_{0} \in\left(0, f u^{0}\right)$ such that $B_{\alpha_{0}}(t) \neq \emptyset$ for all $t \in[0, T]$. It immediately follows from (3.18) and (3.19a,b) for any $t_{a}, t_{b} \in[0, T]$ and for any $\alpha_{1}, \alpha_{2} \in\left(0, \alpha_{0}\right)$ with $\alpha_{1}>\alpha_{2}$ that

$$
\begin{gathered}
y_{1} \in B_{\alpha_{1}}\left(t_{a}\right) \text { and } y_{2} \in \partial B_{\alpha_{2}}\left(t_{b}\right) \text { with } y_{2} \notin \partial \Omega \\
C_{x}\left|y_{2}-y_{1}\right|^{\frac{1}{2}}+C_{t}\left|t_{b}-t_{a}\right|^{\frac{1}{8}} \geq u\left(y_{1}, t_{a}\right)-u\left(y_{2}, t_{b}\right)>\left(\alpha_{1}-\alpha_{2}\right),
\end{gathered}
$$

where $\partial B_{\alpha}(t)$ is the boundary of $B_{\alpha}(t)$. Therefore, (3.20) implies that for any $\alpha \in$ $\left(0, \alpha_{0}\right)$, there exists an $h_{0}(\alpha)$ such that for all $h \leq h_{0}(\alpha)$ and $t \in[0, T]$ there exists a collection of simplices $\mathcal{T}_{\alpha}^{h}(t) \subset \mathcal{T}^{h}$ such that

$$
B_{\alpha}(t) \subset B_{\alpha}^{h}(t):=\cup_{\kappa \in \mathcal{T}_{\alpha}^{h}(t)} \bar{\kappa} \subset B_{\frac{\alpha}{2}}(t) \quad \forall t \in[0, T] .
$$

Similarly, it follows from (3.20) that for any $\alpha \in\left(0, \alpha_{0}\right)$, there exists a $\tau_{0}(\alpha)$ such that for all $\tau \leq \tau_{0}(\alpha)$

$$
B_{\alpha}(t) \subset B_{\frac{\alpha}{2}}\left(t_{n}\right) \subset B_{\frac{\alpha}{4}}(t) \quad \forall t \in\left(t_{n-1}, t_{n}\right], \quad n=1 \rightarrow N .
$$

Clearly, we have from (3.21) and (3.22) that $\alpha_{2}<\alpha_{1}<\alpha_{0}$ implies that $h_{0}\left(\alpha_{2}\right) \leq$ $h_{0}\left(\alpha_{1}\right)$ and $\tau_{0}\left(\alpha_{2}\right) \leq \tau_{0}\left(\alpha_{1}\right)$. For a fixed $\alpha \in\left(0, \alpha_{0}\right)$, it follows from (3.18), (3.5a), and our assumption (iii) of Lemma 3.1 that there exists an $\widehat{h}_{0}(\alpha) \leq h_{0}(\alpha)$ such that for $h \leq \widehat{h}_{0}(\alpha)$

$$
\begin{aligned}
0 & \leq U_{\varepsilon}^{ \pm}(x, t) \leq 2 \alpha & \forall(x, t) \notin B_{\alpha}, \quad \text { and } \quad \tau \leq \tau_{0}(\alpha) . \\
\frac{1}{2} \alpha & \leq U_{\varepsilon}^{ \pm}(x, t) & \forall(x, t) \in B_{\alpha},
\end{aligned}
$$

TheOREM 3.2. Let the assumptions of Lemma 3.1 hold. Then there exist a subsequence of $\left\{U_{\varepsilon}, W_{\varepsilon}, V_{\varepsilon}\right\}_{h}$, where $\left\{U_{\varepsilon}, W_{\varepsilon}, V_{\varepsilon}\right\}$ solve $\left(\mathrm{P}_{\varepsilon}^{h, \tau}\right)$, and functions $\{u, w, v\}$ satisfying $(3.4 \mathrm{a}, \mathrm{b})$ and

$$
w \in L_{l o c}^{2}(\{u>0\}) \quad \text { with } \quad \nabla w \in L_{l o c}^{2}(\{u>0\}),
$$

where $\{u>0\}:=\left\{(x, t) \in \Omega_{T}: u(x, t)>0\right\}$ such that as $h \rightarrow 0$ (3.5a,b), (3.6ac) hold and $W_{\varepsilon}^{+} \rightarrow w, \nabla W_{\varepsilon}^{+} \rightarrow \nabla w$ weakly in $L_{l o c}^{2}(\{u>0\})$. Furthermore, we 
have that $u, v$, and $w$ fulfil $u(\cdot, 0)=u^{0}(\cdot), v(\cdot, 0)=v^{0}(\cdot)$ and are such that for all $\eta, z \in L^{2}\left(0, T ; H^{1}(\Omega)\right)$, with $\operatorname{supp}(z) \subset\{u>0\}$,

$$
\begin{aligned}
& \int_{0}^{T}\left\langle\frac{\partial u}{\partial t}, \eta\right\rangle \mathrm{d} t+\frac{1}{3} \int_{\{u>0\}} u^{3} \nabla w \cdot \nabla \eta \mathrm{d} x \mathrm{~d} t+\frac{1}{2} \int_{\Omega_{T}} u^{2} \nabla v \cdot \nabla \eta \mathrm{d} x \mathrm{~d} t=0, \\
& \int_{\{u>0\}}[c \nabla u \cdot \nabla z-w z] \mathrm{d} x \mathrm{~d} t=0, \\
& \int_{0}^{T}\left\langle\frac{\partial v}{\partial t}, \eta\right\rangle \mathrm{d} t+\int_{\Omega_{T}}[\rho \nabla v \cdot \nabla \eta+u \lambda(v) \nabla v \cdot \nabla \eta] \mathrm{d} x \mathrm{~d} t \\
& \quad+\frac{1}{2} \int_{\{u>0\}} u^{2} \lambda(v) \nabla w \cdot \nabla \eta \mathrm{d} x \mathrm{~d} t=0 .
\end{aligned}
$$

Proof. For any $\eta \in L^{2}\left(0, T ; H^{1}(\Omega)\right)$, we choose $\chi \equiv \pi^{h} \eta$ in (3.3a,c) and now analyze the subsequent terms. First, (2.17), (2.23), (2.18), (1.15) in time, and (3.8) yield for $Z \equiv U_{\varepsilon}$ and $V_{\varepsilon}$, respectively, and for all $\widetilde{\eta} \in H^{1}\left(0, T ; H^{1}(\Omega)\right)$ that

$$
\begin{array}{r}
\left|\int_{0}^{T}\left[\left(\frac{\partial Z}{\partial t}, \pi^{h} \eta\right)^{h}-\left(\frac{\partial Z}{\partial t}, \pi^{h} \eta\right)\right] \mathrm{d} t\right| \leq\left|\int_{0}^{T}\left[\left(\frac{\partial Z}{\partial t}, \pi^{h}[\eta-\widetilde{\eta}]\right)^{h}-\left(\frac{\partial Z}{\partial t}, \pi^{h}[\eta-\widetilde{\eta}]\right)\right] \mathrm{d} t\right| \\
+\mid-\int_{0}^{T}\left(Z, \frac{\partial\left(\pi^{h} \widetilde{\eta}\right)}{\partial t}\right)^{h} \mathrm{~d} t+\left(Z(\cdot, T), \pi^{h} \widetilde{\eta}(\cdot, T)\right)^{h}-\left(Z(\cdot, 0), \pi^{h} \widetilde{\eta}(\cdot, 0)\right)^{h} \\
+\int_{0}^{T}\left(Z, \frac{\partial\left(\pi^{h} \widetilde{\eta}\right)}{\partial t}\right) \mathrm{d} t-\left(Z(\cdot, T), \pi^{h} \widetilde{\eta}(\cdot, T)\right)+\left(Z(\cdot, 0), \pi^{h} \widetilde{\eta}(\cdot, 0)\right) \mid \\
\leq C\left\|\mathcal{G} \frac{\partial Z}{\partial t}\right\|_{L^{2}\left(0, T ; H^{1}(\Omega)\right)}\left\|\pi^{h}[\eta-\widetilde{\eta}]\right\|_{L^{2}\left(0, T ; H^{1}(\Omega)\right)} \\
+C h\|Z\|_{L^{\infty}\left(0, T ; L^{2}(\Omega)\right)}\left\|\pi^{h} \widetilde{\eta}\right\|_{H^{1}\left(0, T ; H^{1}(\Omega)\right)}
\end{array}
$$

$$
\leq C\|\eta-\widetilde{\eta}\|_{L^{2}\left(0, T ; H^{1}(\Omega)\right)}+C h\|\widetilde{\eta}\|_{H^{1}\left(0, T ; H^{1}(\Omega)\right)} .
$$

Furthermore, it follows from (1.16) and (3.8) that

$$
\begin{aligned}
\left|\int_{0}^{T}\left(\frac{\partial Z}{\partial t},\left(I-\pi^{h}\right) \eta\right) \mathrm{d} t\right| & \leq C\left\|\mathcal{G} \frac{\partial Z}{\partial t}\right\|_{L^{2}\left(0, T ; H^{1}(\Omega)\right)}\left\|\left(I-\pi^{h}\right) \eta\right\|_{L^{2}\left(0, T ; H^{1}(\Omega)\right)} \\
& \leq C\left\|\left(I-\pi^{h}\right) \eta\right\|_{L^{2}\left(0, T ; H^{1}(\Omega)\right)} .
\end{aligned}
$$

Combining (3.26), the denseness of $H^{1}\left(0, T ; H^{1}(\Omega)\right)$ in $L^{2}\left(0, T ; H^{1}(\Omega)\right),(3.27),(2.19)$, (1.20), (3.5b), and (3.6a) yields that for $z \equiv u$ and $v$, respectively,

$$
\int_{0}^{T}\left(\frac{\partial Z}{\partial t}, \pi^{h} \eta\right)^{h} \mathrm{~d} t \rightarrow \int_{0}^{T}\left\langle\frac{\partial z}{\partial t}, \eta\right\rangle \mathrm{d} t \quad \text { as } h \rightarrow 0 .
$$

In view of $(2.28),(3.1 \mathrm{~b}),(2.16),(3.7)$, and $(3.8)$, as $\rho>0$, we deduce that

$$
\begin{aligned}
& \left|\int_{0}^{T}\left(\pi^{h}\left[\left(U_{\varepsilon}^{+}\right)^{\frac{1}{2}}\left(U_{\varepsilon}^{-}\right)^{\frac{3}{2}}\right] \Lambda_{\varepsilon}\left(V_{\varepsilon}^{+}\right) \nabla W_{\varepsilon}^{+}, \nabla\left(I-\pi^{h}\right) \eta\right) \mathrm{d} t\right| \\
& \quad \leq\left\|\pi^{h}\left[\left(U_{\varepsilon}^{+}\right)^{\frac{1}{2}}\left(U_{\varepsilon}^{-}\right)^{\frac{3}{2}}\right] \nabla W_{\varepsilon}^{+}\right\|_{L^{2}\left(\Omega_{T}\right)}\left\|\left(I-\pi^{h}\right) \eta\right\|_{L^{2}\left(0, T ; H^{1}(\Omega)\right)} \\
& \quad \leq C\left\|U_{\varepsilon}^{+}\right\|_{L^{\infty}\left(\Omega_{T}\right)}^{\frac{1}{2}}\left\|\left[\pi^{h}\left[\left(U_{\varepsilon}^{-}\right)^{3}\right]\right]^{\frac{1}{2}} \nabla W_{\varepsilon}^{+}\right\|_{L^{2}\left(\Omega_{T}\right)}\left\|\left(I-\pi^{h}\right) \eta\right\|_{L^{2}\left(0, T ; H^{1}(\Omega)\right)} \\
& \quad \leq C\left\|\left(I-\pi^{h}\right) \eta\right\|_{L^{2}\left(0, T ; H^{1}(\Omega)\right)},
\end{aligned}
$$


and similarly

$$
\begin{aligned}
& \left|\int_{0}^{T}\left(\pi^{h}\left[\left(U_{\varepsilon}^{-}\right)^{3}\right] \nabla W_{\varepsilon}^{+}, \nabla\left(I-\pi^{h}\right) \eta\right) \mathrm{d} t\right|+\left|\int_{0}^{T}\left(U_{\varepsilon}^{+} \Lambda_{\varepsilon}\left(V_{\varepsilon}^{+}\right) \nabla V_{\varepsilon}^{+}, \nabla\left(I-\pi^{h}\right) \eta\right) \mathrm{d} t\right| \\
& \quad+\left|\int_{0}^{T}\left(\pi^{h}\left[\left(U_{\varepsilon}^{-}\right)^{2}\right] \nabla V_{\varepsilon}^{-}, \nabla\left(I-\pi^{h}\right) \eta\right) \mathrm{d} t\right|+\left|\int_{0}^{T}\left(\nabla V_{\varepsilon}^{+}, \nabla\left(I-\pi^{h}\right) \eta\right) \mathrm{d} t\right| \\
& \quad+\left|\int_{0}^{T}\left(\nabla U_{\varepsilon}^{+}, \nabla\left(I-\pi^{h}\right) \eta\right) \mathrm{b} t\right| \leq C\left\|\left(I-\pi^{h}\right) \eta\right\|_{L^{2}\left(0, T ; H^{1}(\Omega)\right)} .
\end{aligned}
$$

Noting (3.29b), (2.19), (3.5b), and (3.6a), we have for $Z^{+} \equiv U_{\varepsilon}^{+}$and $V_{\varepsilon}^{+}$, and $z \equiv u$ and $v$, respectively, that

$$
\int_{0}^{T}\left(\nabla Z^{+}, \nabla\left(\pi^{h} \eta\right)\right) \mathrm{d} t \rightarrow \int_{0}^{T}(\nabla z, \nabla \eta) \mathrm{d} t \quad \text { as } h \rightarrow 0 .
$$

It also follows from (3.8), (2.28), (1.13), and (3.4a,b) that for all $\widetilde{\eta} \in L^{\infty}\left(0, T ; W^{1, \infty}(\Omega)\right)$

$$
\begin{aligned}
& \left|\int_{0}^{T}\left(\left(\pi^{h}\left[\left(U_{\varepsilon}^{-}\right)^{2}\right]-u^{2}\right) \nabla V_{\varepsilon}^{-}, \nabla \eta\right) \mathrm{d} t\right| \\
& \leq\left\|\pi^{h}\left[\left(U_{\varepsilon}^{-}\right)^{2}\right]-u^{2}\right\|_{L^{\infty}\left(\Omega_{T}\right)}\left\|V_{\varepsilon}^{-}\right\|_{L^{2}\left(0, T ; H^{1}(\Omega)\right)}\|\eta\|_{L^{2}\left(0, T ; H^{1}(\Omega)\right)} \\
& \text { (3.31a) } \\
& \leq C\left[\left\|\pi^{h}\left[\left(U_{\varepsilon}^{-}\right)^{2}-u^{2}\right]\right\|_{L^{\infty}\left(\Omega_{T}\right)}+\left\|\left(I-\pi^{h}\right) u^{2}\right\|_{L^{\infty}\left(\Omega_{T}\right)}\right]\|\eta\|_{L^{2}\left(0, T ; H^{1}(\Omega)\right)}, \\
& \left|\int_{0}^{T}\left(\left(U_{\varepsilon}^{+} \Lambda_{\varepsilon}\left(V_{\varepsilon}^{+}\right)-u \lambda(v)\right) \nabla V_{\varepsilon}^{+}, \nabla \eta\right) \mathrm{d} t\right| \\
& \leq\left|\int_{0}^{T}\left(\left(U_{\varepsilon}^{+}-u\right) \Lambda_{\varepsilon}\left(V_{\varepsilon}^{+}\right) \nabla V_{\varepsilon}^{+}, \nabla \eta\right) \mathrm{d} t\right| \\
& +\left|\int_{0}^{T}\left[\left(u\left(\Lambda_{\varepsilon}\left(V_{\varepsilon}^{+}\right)-\lambda(v)\right) \nabla V_{\varepsilon}^{+}, \nabla(\eta-\widetilde{\eta})\right)+\left(u\left(\Lambda_{\varepsilon}\left(V_{\varepsilon}^{+}\right)-\lambda(v)\right) \nabla V_{\varepsilon}^{+}, \nabla \widetilde{\eta}\right)\right] \mathrm{d} t\right| \\
& \leq\left\|U_{\varepsilon}^{+}-u\right\|_{L^{\infty}\left(\Omega_{T}\right)}\left\|V_{\varepsilon}^{+}\right\|_{L^{2}\left(0, T ; H^{1}(\Omega)\right)}\|\eta\|_{L^{2}\left(0, T ; H^{1}(\Omega)\right)} \\
& +\|u\|_{L^{\infty}\left(\Omega_{T}\right)}\left\|\Lambda_{\varepsilon}\left(V_{\varepsilon}^{+}\right)-\lambda(v)\right\|_{L^{\infty}\left(\Omega_{T}\right)}\left\|V_{\varepsilon}^{+}\right\|_{L^{2}\left(0, T ; H^{1}(\Omega)\right)}\|\eta-\widetilde{\eta}\|_{L^{2}\left(0, T ; H^{1}(\Omega)\right)} \\
& +\|u\|_{L^{\infty}\left(\Omega_{T}\right)}\left\|\Lambda_{\varepsilon}\left(V_{\varepsilon}^{+}\right)-\lambda(v)\right\|_{L^{2}\left(\Omega_{T}\right)}\left\|V_{\varepsilon}^{+}\right\|_{L^{2}\left(0, T ; H^{1}(\Omega)\right)}\|\widetilde{\eta}\|_{L^{\infty}\left(0, T ; W^{1, \infty}(\Omega)\right)} \\
& \leq C\left[\left\|U_{\varepsilon}^{+}-u\right\|_{L^{\infty}\left(\Omega_{T}\right)}\|\eta\|_{L^{2}\left(0, T ; H^{1}(\Omega)\right)}+\|\eta-\widetilde{\eta}\|_{L^{2}\left(0, T ; H^{1}(\Omega)\right)}\right. \\
& \left.+\left\|\Lambda_{\varepsilon}\left(V_{\varepsilon}^{+}\right)-\lambda(v)\right\|_{L^{2}\left(\Omega_{T}\right)}\|\widetilde{\eta}\|_{L^{\infty}\left(0, T ; W^{1, \infty}(\Omega)\right)}\right] .
\end{aligned}
$$

Noting that $L^{\infty}\left(0, T ; W^{1, \infty}(\Omega)\right)$ is dense in $L^{2}\left(0, T ; H^{1}(\Omega)\right),(3.29 \mathrm{~b}),(2.19),(3.31 \mathrm{a}, \mathrm{b})$, (3.5a), (2.13), (3.6b), and (3.6a), we have that

$$
\begin{array}{cl}
\int_{0}^{T}\left(\pi^{h}\left[\left(U_{\varepsilon}^{-}\right)^{2}\right] \nabla V_{\varepsilon}^{-}, \nabla\left(\pi^{h} \eta\right)\right) \mathrm{d} t \rightarrow \int_{0}^{T}\left(u^{2} \nabla v, \nabla \eta\right) \mathrm{d} t & \text { as } h \rightarrow 0 \\
\int_{0}^{T}\left(U_{\varepsilon}^{+} \Lambda_{\varepsilon}\left(V_{\varepsilon}^{+}\right) \nabla V_{\varepsilon}^{+}, \nabla\left(\pi^{h} \eta\right)\right) \mathrm{d} t \rightarrow \int_{0}^{T}(u \lambda(v) \nabla v, \nabla \eta) \mathrm{d} t & \text { as } h \rightarrow 0 .
\end{array}
$$


We now show the compactness of $\left\{W_{\varepsilon}^{+}\right\}_{h}$ on compact subsets of $\{u>0\}$. On noting (3.23), (3.8), (2.28), and (2.16), we have for all $h \leq \widehat{h}_{0}(\alpha)$, similarly to (3.29a), that

$$
\begin{aligned}
& \left|\int_{\Omega_{T} \backslash B_{\alpha}} \pi^{h}\left[\left(U_{\varepsilon}^{+}\right)^{\frac{1}{2}}\left(U_{\varepsilon}^{-}\right)^{\frac{3}{2}}\right] \Lambda_{\varepsilon}\left(V_{\varepsilon}^{+}\right) \nabla W_{\varepsilon}^{+} \cdot \nabla \eta \mathrm{d} x \mathrm{~d} t\right| \\
& \quad \leq C\left\|U_{\varepsilon}^{+}\right\|_{L^{\infty}\left(\Omega_{T} \backslash B_{\alpha}\right)}^{\frac{1}{2}}\left\|\left[\pi^{h}\left[\left(U_{\varepsilon}^{-}\right)^{3}\right]\right]^{\frac{1}{2}} \nabla W_{\varepsilon}^{+}\right\|_{L^{2}\left(\Omega_{T}\right)}\|\eta\|_{L^{2}\left(0, T ; H^{1}(\Omega)\right)} \\
& \quad \leq C \alpha^{\frac{1}{2}}\|\eta\|_{L^{2}\left(0, T ; H^{1}(\Omega)\right)}
\end{aligned}
$$

and similarly

$$
\left|\int_{\Omega_{T} \backslash B_{\alpha}} \pi^{h}\left[\left(U_{\varepsilon}^{-}\right)^{3}\right] \nabla W_{\varepsilon}^{+} . \nabla \eta \mathrm{d} x \mathrm{~d} t\right| \leq C \alpha^{\frac{3}{2}}\|\eta\|_{L^{2}\left(0, T ; H^{1}(\Omega)\right)} .
$$

It follows from (3.23), (3.21), and (3.8) that for all $h \leq \widehat{h}_{0}\left(\frac{\alpha}{8}\right)$

$$
\begin{aligned}
C_{1} \alpha^{3} \int_{B_{\frac{\alpha}{4}}}\left|\nabla W_{\varepsilon}^{+}\right|^{2} \mathrm{~d} x \mathrm{~d} t & \leq C_{1} \alpha^{3} \int_{B_{\frac{\alpha}{4}}^{h}}\left|\nabla W_{\varepsilon}^{+}\right|^{2} \mathrm{~d} x \mathrm{~d} t \leq \int_{B_{\frac{\alpha}{4}}^{h}} \pi^{h}\left[\left(U_{\varepsilon}^{-}\right)^{3}\right]\left|\nabla W_{\varepsilon}^{+}\right|^{2} \mathrm{~d} x \mathrm{~d} t \\
& \leq C,
\end{aligned}
$$

where $B_{\alpha}^{h}:=\left\{(x, t) \in \bar{\Omega}_{T}: x \in B_{\alpha}^{h}(t)\right\}$. Similarly to (3.31a,b), it follows from (2.28), (3.34), (1.13), and (3.4a,b) that for all $h \leq \widehat{h}_{0}\left(\frac{\alpha}{8}\right)$ and for all $\widetilde{\eta} \in L^{\infty}\left(0, T ; W^{1, \infty}(\Omega)\right)$

$$
\begin{aligned}
& \left|\int_{B_{\alpha}}\left(\pi^{h}\left[\left(U_{\varepsilon}^{-}\right)^{3}\right]-u^{3}\right) \nabla W_{\varepsilon}^{+} \cdot \nabla \eta \mathrm{d} x \mathrm{~d} t\right| \\
& \leq\left\|\pi^{h}\left[\left(U_{\varepsilon}^{-}\right)^{3}\right]-u^{3}\right\|_{L^{\infty}\left(\Omega_{T}\right)}\left\|\nabla W_{\varepsilon}^{+}\right\|_{L^{2}\left(B_{\alpha}\right)}\|\eta\|_{L^{2}\left(0, T ; H^{1}(\Omega)\right)}
\end{aligned}
$$

$$
\begin{aligned}
& \leq C \alpha^{-\frac{3}{2}}\left[\left\|\pi^{h}\left[\left(U_{\varepsilon}^{-}\right)^{3}-u^{3}\right]\right\|_{L^{\infty}\left(\Omega_{T}\right)}+\left\|\left(I-\pi^{h}\right) u^{3}\right\|_{L^{\infty}\left(\Omega_{T}\right)}\right]\|\eta\|_{L^{2}\left(0, T ; H^{1}(\Omega)\right)}, \\
& \left|\int_{B_{\alpha}}\left(\pi^{h}\left[\left(U_{\varepsilon}^{+}\right)^{\frac{1}{2}}\left(U_{\varepsilon}^{-}\right)^{\frac{3}{2}}\right] \Lambda_{\varepsilon}\left(V_{\varepsilon}^{+}\right)-u^{2} \lambda(v)\right) \nabla W_{\varepsilon}^{+} \cdot \nabla \eta \mathrm{d} x \mathrm{~d} t\right| \\
& \leq\left|\int_{B_{\alpha}}\left(\pi^{h}\left[\left(U_{\varepsilon}^{+}\right)^{\frac{1}{2}}\left(U_{\varepsilon}^{-}\right)^{\frac{3}{2}}\right]-u^{2}\right) \Lambda_{\varepsilon}\left(V_{\varepsilon}^{+}\right) \nabla W_{\varepsilon}^{+} \cdot \nabla \eta \mathrm{d} x \mathrm{~d} t\right| \\
& \quad+\left|\int_{B_{\alpha}} u^{2}\left(\Lambda_{\varepsilon}\left(V_{\varepsilon}^{+}\right)-\lambda(v)\right) \nabla W_{\varepsilon}^{+} \cdot[\nabla(\eta-\widetilde{\eta})+\nabla \widetilde{\eta}] \mathrm{d} x \mathrm{~d} t\right| \\
& \leq\left\|\pi^{h}\left[\left(U_{\varepsilon}^{+}\right)^{\frac{1}{2}}\left(U_{\varepsilon}^{-}\right)^{\frac{3}{2}}\right]-u^{2}\right\|_{L^{\infty}\left(\Omega_{T}\right)}\left\|\nabla W_{\varepsilon}^{+}\right\|_{L^{2}\left(B_{\alpha}\right)}\|\eta\|_{L^{2}\left(0, T ; H^{1}(\Omega)\right)} \\
& \quad+\left\|u^{2}\right\|_{L^{\infty}\left(\Omega_{T}\right)}\left\|\Lambda_{\varepsilon}\left(V_{\varepsilon}^{+}\right)-\lambda(v)\right\|_{L^{\infty}\left(\Omega_{T}\right)}\left\|\nabla W_{\varepsilon}^{+}\right\|_{L^{2}\left(B_{\alpha}\right)}\|\eta-\widetilde{\eta}\|_{L^{2}\left(0, T ; H^{1}(\Omega)\right)}\left\|W_{\varepsilon}^{+}\right\|_{L^{2}\left(B_{\alpha}\right)}\|\widetilde{\eta}\|_{L^{\infty}\left(0, T ; W^{1, \infty}(\Omega)\right)} \\
& \left.\quad+\left\|u^{2}\right\|_{L^{\infty}\left(\Omega_{T}\right)}\left\|\Lambda_{\varepsilon}\left(V_{\varepsilon}^{+}\right)-\lambda(v)\right\|_{L^{2}\left(\Omega_{T}\right)}\|\nabla\|^{h}\right) u^{2}\left\|_{L^{\infty}\left(\Omega_{T}\right)}\right\| \eta \|_{L^{2}\left(0, T ; H^{1}(\Omega)\right)} \\
& \leq C \alpha^{-\frac{3}{2}}\left[\| \pi^{h}\left[\left(U_{\varepsilon}^{+}\right)^{\frac{1}{2}}\left(U_{\varepsilon}^{-}\right)^{\frac{3}{2}}-u^{2}\right]-\left(I-\pi^{h}\right)\right.
\end{aligned}
$$

$$
\left.+\left\|\Lambda_{\varepsilon}\left(V_{\varepsilon}^{+}\right)-\lambda(v)\right\|_{L^{2}\left(\Omega_{T}\right)}\|\widetilde{\eta}\|_{L^{\infty}\left(0, T ; W^{1, \infty}(\Omega)\right)}+\|\eta-\widetilde{\eta}\|_{L^{2}\left(0, T ; H^{1}(\Omega)\right)}\right] .
$$

From $(3.23)$ we have for all $h \leq \widehat{h}_{0}\left(\frac{\alpha}{8}\right)$ and for a.e. $t \in(0, T)$ that $\xi^{h}(\cdot, t):=U_{\varepsilon}^{+}(\cdot, t) \pm$ $\frac{\alpha}{16} \zeta^{h}(\cdot, t) /\left\|\zeta^{h}(\cdot, t)\right\|_{L^{\infty}(\Omega)} \in K^{h}$ for any $\zeta^{h} \in L^{2}\left(0, T ; S^{h}\right)$ with $\operatorname{supp}\left(\zeta^{h}\right) \subset B_{\frac{\alpha}{8}}$. 
Choosing $z^{h} \equiv \xi^{h}$ in (3.3b) yields, as $\phi^{-} \equiv 0$, for all $h \leq \widehat{h}_{0}\left(\frac{\alpha}{8}\right)$ that

(3.36)

$\int_{0}^{T}\left[c\left(\nabla U_{\varepsilon}^{+}, \nabla \zeta^{h}\right)-\left(W_{\varepsilon}^{+}, \zeta^{h}\right)^{h}\right] \mathrm{d} t=0 \quad \forall \zeta^{h} \in L^{2}\left(0, T ; S^{h}\right) \quad$ with $\operatorname{supp}\left(\zeta^{h}\right) \subset B_{\frac{\alpha}{8}}$.

Next we derive a bound on $W_{\varepsilon}^{+}$locally on the set $\{u>0\}$. For any $\alpha \in\left(0, \alpha_{0}\right)$ and any $t \in[0, T]$, we choose a cut-off function $\theta_{\alpha}(\cdot, t) \in C^{\infty}(\bar{\Omega})$ such that

$$
\begin{array}{ll}
\theta_{\alpha}(\cdot, t) \equiv 1 & \text { on } B_{\alpha}(t), \quad 0 \leq \theta_{\alpha}(\cdot, t) \leq 1 \quad \text { on } B_{\frac{\alpha}{2}}(t) \backslash B_{\alpha}(t), \\
\theta_{\alpha}(\cdot, t) \equiv 0 & \text { on } \bar{\Omega} \backslash B_{\frac{\alpha}{2}}(t) \quad \text { and } \quad\left|\nabla \theta_{\alpha}(\cdot, t)\right| \leq C \alpha^{-2} .
\end{array}
$$

It follows from (3.20) that this last property can be achieved. We have from (3.37) and (3.21) that

$$
\operatorname{supp}\left(\pi^{h}\left[\theta_{\frac{\alpha}{2}}^{2} W_{\varepsilon}^{+}\right]\right) \subset B_{\frac{\alpha}{4}}^{h} \subset B_{\frac{\alpha}{8}} \quad \forall h \leq \widehat{h}_{0}\left(\frac{\alpha}{8}\right) .
$$

Next we note, as $d=1$, that for any $\kappa=\left(p_{j}, p_{j+1}\right) \in \mathcal{T}^{h}$ and any $z_{1}, z_{2} \in C(\bar{\kappa})$

$$
\nabla \pi^{h}\left[z_{1}^{2} z_{2}\right]=\left[\left(z_{1} z_{2}\right)\left(p_{j}\right)+\left(z_{1} z_{2}\right)\left(p_{j+1}\right)\right] \nabla \pi^{h}\left[z_{1}\right]+z_{1}\left(p_{j}\right) z_{1}\left(p_{j+1}\right) \nabla \pi^{h}\left[z_{2}\right] \quad \text { on } \kappa \text {. }
$$

It follows from (2.1), (3.36), (3.38), (3.39), (3.37), (3.21), and (1.19) that for all $h \leq \widehat{h}_{0}\left(\frac{\alpha}{8}\right)$

$$
\begin{array}{r}
\int_{\Omega_{T}} \pi^{h}\left[\left(\theta_{\frac{\alpha}{2}} W_{\varepsilon}^{+}\right)^{2}\right] \mathrm{d} x \mathrm{~d} t=\int_{0}^{T}\left(W_{\varepsilon}^{+}, \pi^{h}\left[\theta_{\frac{\alpha}{2}}^{2} W_{\varepsilon}^{+}\right]\right)^{h} \mathrm{~d} t=\int_{0}^{T} c\left(\nabla U_{\varepsilon}^{+}, \nabla\left(\pi^{h}\left[\theta_{\frac{\alpha}{2}}^{2} W_{\varepsilon}^{+}\right]\right)\right) \mathrm{d} t \\
\leq C\left\|U_{\varepsilon}^{+}\right\|_{L^{2}\left(0, T ; H^{1}(\Omega)\right)}\left[\left\|\nabla \theta_{\frac{\alpha}{2}}\right\|_{L^{\infty}\left(\Omega_{T}\right)}\left[\int_{\Omega_{T}} \pi^{h}\left[\left(\theta_{\frac{\alpha}{2}} W_{\varepsilon}^{+}\right)^{2}\right] \mathrm{d} x \mathrm{~d} t\right]^{\frac{1}{2}}+\left\|\nabla W_{\varepsilon}^{+}\right\|_{L^{2}\left(B_{\frac{\alpha}{4}}^{h}\right)}\right]
\end{array}
$$

$$
\leq C\left(1+\alpha^{-4}\right)\left\|U_{\varepsilon}^{+}\right\|_{L^{2}\left(0, T ; H^{1}(\Omega)\right)}^{2}+C\left\|\nabla W_{\varepsilon}^{+}\right\|_{L^{2}\left(B_{\frac{\alpha}{4}}^{h}\right)}^{2} .
$$

From (3.21), (3.37), and (2.14), we obtain that for all $h \leq \widehat{h}_{0}\left(\frac{\alpha}{8}\right)$

$$
\int_{\Omega_{T}} \pi^{h}\left[\left(\theta_{\frac{\alpha}{2}} W_{\varepsilon}^{+}\right)^{2}\right] \mathrm{d} x \mathrm{~d} t \geq \int_{B_{\alpha}^{h}} \pi^{h}\left[\left(W_{\varepsilon}^{+}\right)^{2}\right] \mathrm{d} x \mathrm{~d} t \geq \int_{B_{\alpha}^{h}}\left(W_{\varepsilon}^{+}\right)^{2} \mathrm{~d} x \mathrm{~d} t \geq\left\|W_{\varepsilon}^{+}\right\|_{L^{2}\left(B_{\alpha}\right)}^{2} .
$$

Therefore, combining (3.34), (3.40), (3.41), (3.9), and (3.8) yields that

$$
\left\|W_{\varepsilon}^{+}\right\|_{L^{2}\left(0, T ; H^{1}\left(B_{\alpha}(t)\right)\right)} \leq C\left(\alpha^{-1}\right) \quad \forall h \leq \widehat{h}_{0}\left(\frac{\alpha}{8}\right) .
$$

The bound (3.42) implies the existence of a subsequence and a function $w \in$ $L^{2}\left(0, T ; H^{1}\left(B_{\alpha}(t)\right)\right)$ such that

$$
W_{\varepsilon}^{+} \rightarrow w, \quad \nabla W_{\varepsilon}^{+} \rightarrow \nabla w \quad \text { weakly in } L^{2}\left(B_{\alpha}\right) \quad \text { as } h \rightarrow 0
$$

On noting that $L^{\infty}\left(0, T ; W^{1, \infty}(\Omega)\right)$ is dense in $L^{2}\left(0, T ; H^{1}(\Omega)\right)$, (3.29a,b), (2.19), (3.35a,b), (3.5a), (2.13), (3.6b), and (3.43), we have that as $h \rightarrow 0$

$$
\int_{B_{\alpha}} \pi^{h}\left[\left(U_{\varepsilon}^{-}\right)^{3}\right] \nabla W_{\varepsilon}^{+} \cdot \nabla\left(\pi^{h} \eta\right) \mathrm{d} x \mathrm{~d} t \rightarrow \int_{B_{\alpha}} u^{3} \nabla w \cdot \nabla \eta \mathrm{d} x \mathrm{~d} t
$$

$$
\int_{B_{\alpha}} \pi^{h}\left[\left(U_{\varepsilon}^{+}\right)^{\frac{1}{2}}\left(U_{\varepsilon}^{-}\right)^{\frac{3}{2}}\right] \Lambda_{\varepsilon}\left(V_{\varepsilon}^{+}\right) \nabla W_{\varepsilon}^{+} \cdot \nabla\left(\pi^{h} \eta\right) \mathrm{d} x \mathrm{~d} t \rightarrow \int_{B_{\alpha}} u^{2} \lambda(v) \nabla w \cdot \nabla \eta \mathrm{d} x \mathrm{~d} t .
$$


Using (2.1), (2.18), and (3.42), we deduce for all $\zeta \in L^{2}\left(0, T ; H^{1}(\Omega)\right)$ with $\operatorname{supp}(\zeta) \subset$ $B_{\alpha}$ and for all $h \leq \widehat{h}_{0}\left(\frac{\alpha}{8}\right)$ that

$$
\begin{aligned}
\left|\int_{0}^{T}\left[\left(W_{\varepsilon}^{+}, \pi^{h} \zeta\right)^{h}-\left(W_{\varepsilon}^{+}, \zeta\right)\right] \mathrm{d} t\right| & =\left|\int_{\Omega_{T}}\left(I-\pi^{h}\right)\left(W_{\varepsilon}^{+} \zeta\right) \mathrm{d} x \mathrm{~d} t\right| \\
& \leq C h \int_{\Omega_{T}}\left|\nabla\left(W_{\varepsilon}^{+} \zeta\right)\right| \mathrm{d} x \mathrm{~d} t \\
& \leq C h\left\|W_{\varepsilon}^{+}\right\|_{L^{2}\left(0, T ; H^{1}\left(B_{\alpha}(t)\right)\right)}\|\zeta\|_{L^{2}\left(0, T ; H^{1}(\Omega)\right)} \\
& \leq C\left(\alpha^{-1}\right) h\|\zeta\|_{L^{2}\left(0, T ; H^{1}(\Omega)\right)}
\end{aligned}
$$

It follows from (3.45) and (3.43) that for all $\zeta \in L^{2}\left(0, T ; H^{1}(\Omega)\right)$ with $\operatorname{supp}(\zeta) \subset B_{\alpha}$,

$$
\int_{0}^{T}\left(W_{\varepsilon}^{+}, \pi^{h} \zeta\right)^{h} \mathrm{~d} t \rightarrow \int_{0}^{T}(w, \zeta) \mathrm{d} t=\int_{B_{\alpha}} w \zeta \mathrm{d} x \mathrm{~d} t \quad \text { as } h \rightarrow 0 .
$$

Combining (3.30) for $u$ and (3.46) and noting (3.36) yield that

$$
\int_{B_{\alpha}}[c \nabla u \cdot \nabla \zeta-w \zeta] \mathrm{d} x \mathrm{~d} t=0 \quad \forall \zeta \in L^{2}\left(0, T ; H^{1}(\Omega)\right) \text { with } \operatorname{supp}(\zeta) \subset B_{\alpha}
$$

This uniquely defines $w$ in terms of $u$ on the set $B_{\alpha}$. Repeating (3.44a,b) for all $\alpha>0$ and noting $(3.33 \mathrm{a}, \mathrm{b})$ and $(2.19)$ yield for all $\eta \in L^{2}\left(0, T ; H^{1}(\Omega)\right)$ that as $h \rightarrow 0$

$$
\begin{aligned}
\int_{\Omega_{T}} \pi^{h}\left[\left(U_{\varepsilon}^{-}\right)^{3}\right] \nabla W_{\varepsilon}^{+} \cdot \nabla\left(\pi^{h} \eta\right) \mathrm{d} x \mathrm{~d} t & \rightarrow \int_{B_{0}} u^{3} \nabla w \cdot \nabla \eta \mathrm{d} x \mathrm{~d} t \\
\int_{\Omega_{T}} \pi^{h}\left[\left(U_{\varepsilon}^{+}\right)^{\frac{1}{2}}\left(U_{\varepsilon}^{-}\right)^{\frac{3}{2}}\right] \Lambda_{\varepsilon}\left(V_{\varepsilon}^{+}\right) \nabla W_{\varepsilon}^{+} \cdot \nabla\left(\pi^{h} \eta\right) \mathrm{d} x \mathrm{~d} t & \rightarrow \int_{B_{0}} u^{2} \lambda(v) \nabla w \cdot \nabla \eta \mathrm{d} x \mathrm{~d} t
\end{aligned}
$$

Combining (3.3a,c), (3.28), (3.30), (3.32a,b), and (3.48a,b) and repeating (3.47) for all $\alpha>0$ yield that the functions $\{u, w, v\}$ satisfy $(3.4 \mathrm{a}, \mathrm{b}),(3.24)$, and $(3.25 \mathrm{a}-\mathrm{c})$.

Remark 3.3. If $v^{0} \equiv 0$, then $(3.25 \mathrm{a}-\mathrm{c})$ collapses to

$$
\int_{0}^{T}\left\langle\frac{\partial u}{\partial t}, \eta\right\rangle \mathrm{d} t-\frac{c}{3} \int_{\{u>0\}} u^{3} \nabla(\Delta u) . \nabla \eta \mathrm{d} x \mathrm{~d} t=0 \quad \forall \eta \in L^{2}\left(0, T ; H^{1}(\Omega)\right)
$$

since $v \equiv \lambda(v) \equiv 0$ in $\Omega_{T}$ and $w \equiv-c \Delta u$ on $\{u>0\}$. This is the Bernis-Friedman weak formulation of the degenerate fourth order equation $\frac{\partial u}{\partial t}+\frac{c}{3} \nabla \cdot\left(u^{3} \nabla(\Delta u)\right)=0$; see [9]. Note that (3.49) incorporates a weak formulation of the boundary condition $\frac{c}{3} u^{3} \frac{\partial \Delta u}{\partial \nu_{\partial \Omega}}=0$. In addition, (3.25b) implies that $\frac{\partial u}{\partial \nu_{\partial \Omega}}(x, t)=0$ for $(x, t) \in \partial \Omega \times(0, T)$ whenever $u(x, t)>0$. Therefore, $(3.25 \mathrm{a}-\mathrm{c})$ is the natural extension of the BernisFriedman weak formulation to the problem $(\mathrm{P})$ in the presence of surfactant $\left(v^{0} \not \equiv 0\right)$.

Remark 3.4. The obstacle formulation in $\left(\mathrm{P}_{\varepsilon}^{h, \tau}\right)$ is not crucial in proving wellposedness and convergence of the resulting approximation $\left\{U_{\varepsilon}, W_{\varepsilon}, V_{\varepsilon}\right\}$ to a solution, 
$\{u, w, v\}$, of (P). Replacing $\pi^{h}\left[\left(U_{\varepsilon}^{n-1}\right)^{3}\right], \pi^{h}\left[\left(U_{\varepsilon}^{n-1}\right)^{2}\right]$ by $\pi^{h}\left[\left[U_{\varepsilon}^{n-1}\right]_{+}^{3}\right], \pi^{h}\left[\left[U_{\varepsilon}^{n-1}\right]_{+}^{2}\right]$ in (2.11a), the inequality by an equality, $K^{h}$ by $S^{h}$ in $(2.11 \mathrm{~b})$, and $U_{\varepsilon}^{n} \Lambda_{\varepsilon}\left(V_{\varepsilon}^{n}\right)$, $\pi^{h}\left[\left(U_{\varepsilon}^{n}\right)^{\frac{1}{2}}\left(U_{\varepsilon}^{n-1}\right)^{\frac{3}{2}}\right]$ by $\pi^{h}\left[\left[U_{\varepsilon}^{n}\right]_{+}\right] \Lambda_{\varepsilon}\left(V_{\varepsilon}^{n}\right), \pi^{h}\left[\left[U_{\varepsilon}^{n}\right]_{+}^{\frac{1}{2}}\left[U_{\varepsilon}^{n-1}\right]_{+}^{\frac{3}{2}}\right]$ in $(2.11 \mathrm{c})$, one can easily adapt the proofs of Theorems 2.2, 2.4, and 3.2 and Lemmas 2.3, 2.5, and 3.1. Hence one can pass to a limit $\{u, w, v\}$ which solves $(\mathrm{P})$ in the sense of $(3.25 \mathrm{a}-\mathrm{c})$ with $u^{2}$ replaced by $[u]_{+}^{2}$ in (3.25a) and $u \lambda(v)$ replaced by $[u]_{+} \lambda(v)$ in $(3.25 \mathrm{c})$. Using $[u]_{-}$as a test function in the modified (3.25a), one recovers the nonnegativity of $u$ and hence the weak formulation $(3.25 \mathrm{a}-\mathrm{c})$. However, as $U_{\varepsilon}^{n}(\cdot)$ can now become negative in many disconnected regions where $u\left(\cdot, t_{n}\right) \equiv 0$, this makes the location of the approximate free boundary more difficult.

Remark 3.5. On choosing $U_{\varepsilon}^{0} \equiv \pi^{h} u^{0}$ and $V_{\varepsilon}^{0} \equiv \pi^{h} v^{0}$, we need the quasi uniformity assumption on the partitioning $\mathcal{T}^{h}$ only in order to obtain the bound (2.48b) via (2.51), (2.52), and (2.21) and the bound (3.26) via (2.23). However, we can replace this with the far milder assumption that $\left\{\mathcal{T}^{h}\right\}_{h>0}$ is a regular partitioning at the expense of a minimum time step constraint as in [4]. It is easily established from (1.16), (2.22), $\left\{\mathcal{T}^{h}\right\}_{h>0}$ being a regular partitioning, elliptic regularity, assuming that $\Omega$ is convex polygonal if $d=2$, and (2.17) that

$$
\left\|\left(\mathcal{G}-\mathcal{G}^{h}\right) z^{h}\right\|_{1} \leq C h\left\|z^{h}\right\|_{0} \quad \forall z^{h} \in Z^{h} .
$$

Then choosing $\chi \equiv \mathcal{G}^{h}\left[\frac{U_{\varepsilon}^{n}-U_{\varepsilon}^{n-1}}{\tau_{n}}\right]$ and $\chi \equiv \mathcal{G}^{h}\left[\frac{V_{\varepsilon}^{n}-V_{\varepsilon}^{n-1}}{\tau_{n}}\right]$ in (2.11a) and (2.11c), respectively, we obtain, similarly to $(2.51)$ and $(2.52)$, that

$$
\left\|\mathcal{G}^{h} \frac{\partial U_{\varepsilon}}{\partial t}\right\|_{L^{2}\left(0, T ; H^{1}(\Omega)\right)}+\left\|\mathcal{G}^{h} \frac{\partial V_{\varepsilon}}{\partial t}\right\|_{L^{2}\left(0, T ; H^{1}(\Omega)\right)} \leq C\left(\left\|U_{\varepsilon}\right\|_{L^{\infty}\left(\Omega_{T}\right)}\right)
$$

on noting (2.53). Combining (3.50) and (3.51) and noting the fifth and sixth bound in (2.48a), it follows for $Z \equiv U_{\varepsilon}$ or $V_{\varepsilon}$ that

$$
\begin{aligned}
\left\|\mathcal{G} \frac{\partial Z}{\partial t}\right\|_{L^{2}\left(0, T ; H^{1}(\Omega)\right)} & \leq\left\|\left(\mathcal{G}-\mathcal{G}^{h}\right) \frac{\partial Z}{\partial t}\right\|_{L^{2}\left(0, T ; H^{1}(\Omega)\right)}+\left\|\mathcal{G}^{h} \frac{\partial Z}{\partial t}\right\|_{L^{2}\left(0, T ; H^{1}(\Omega)\right)} \\
& \leq C h\left\|\frac{\partial Z}{\partial t}\right\|_{L^{2}\left(\Omega_{T}\right)}+C\left(\left\|U_{\varepsilon}\right\|_{L^{\infty}\left(\Omega_{T}\right)}\right) \\
& \leq C\left(\left\|U_{\varepsilon}\right\|_{L^{\infty}\left(\Omega_{T}\right)}\right)\left(\tau_{\min }^{-\frac{1}{2}} h+1\right) \leq C\left(\left\|U_{\varepsilon}\right\|_{L^{\infty}\left(\Omega_{T}\right)}\right)
\end{aligned}
$$

if the mild time step constraint $C h^{2} \leq \tau_{\min }:=\min _{n=1 \rightarrow N} \tau_{n}$ is satisfied.

3.1. Inclusion of van der Waals forces. We now extend Lemma 3.1 and Theorem 3.2 to the approximation $\left(\mathrm{P}_{\delta, \varepsilon}^{h, \tau}\right)$.

Lemma 3.6. Let $d=1, \rho>0$ and $u^{0}, v^{0} \in K$, with $u^{0}(x) \geq \zeta>0$ for all $x \in \Omega$. Let $\left\{\mathcal{T}^{h}, U_{\varepsilon}^{0}, V_{\varepsilon}^{0}, \tau, \varepsilon\right\}_{h>0}$ be such that assumptions (i), (ii), and (iii) of Lemma 3.1 hold. Then there exist a subsequence of $\left\{U_{\varepsilon}, V_{\varepsilon}\right\}_{h}$, where $\left\{U_{\varepsilon}, W_{\varepsilon}, V_{\varepsilon}\right\}$ solve $\left(\mathrm{P}_{\delta, \varepsilon}^{h, \tau}\right)$, and functions $\{u, v\}$ satisfying $(3.4 \mathrm{a}, \mathrm{b})$ with $u(x, 0)=u^{0}(x)$ for all $x \in \bar{\Omega}, v(\cdot, 0)=$ $v^{0}(\cdot)$ in $\left(H^{1}(\Omega)\right)^{\prime}, f u(\cdot, t)=f u^{0}>0$ for all $t \in[0, T]$, and $f v(\cdot, t)=f v^{0}$ for a.e. $t \in[0, T]$, such that as $h \rightarrow 0(3.5 \mathrm{a}, \mathrm{b})$ and $(3.6 \mathrm{a}-\mathrm{c})$ hold.

Proof. The proof is exactly the same as that of Lemma 3.1.

TheOREM 3.7. Let the assumptions of Lemma 3.6 hold. Then there exist a subsequence of $\left\{U_{\varepsilon}, W_{\varepsilon}, V_{\varepsilon}\right\}_{h}$, where $\left\{U_{\varepsilon}, W_{\varepsilon}, V_{\varepsilon}\right\}$ solve $\left(\mathrm{P}_{\delta, \varepsilon}^{h, \tau}\right)$, and functions $\{u, w, v\}$ satisfying (3.4a,b), $w \in L^{2}\left(0, T ; H^{1}(\Omega)\right)$, and $u>0$ on $\bar{\Omega}_{T}$. In addition, as $h \rightarrow 0$, $(3.5 \mathrm{a}, \mathrm{b}),(3.6 \mathrm{a}-\mathrm{c})$, and $W_{\varepsilon}^{+} \rightarrow w$ weakly in $L^{2}\left(0, T ; H^{1}(\Omega)\right)$ hold. Furthermore, we 
have that $u, v$, and $w$ fulfil $u(\cdot, 0)=u^{0}(\cdot), v(\cdot, 0)=v^{0}(\cdot)$ and are such that for all $\eta \in L^{2}\left(0, T ; H^{1}(\Omega)\right)$,

$$
\begin{aligned}
& \int_{0}^{T}\left\langle\frac{\partial u}{\partial t}, \eta\right\rangle \mathrm{d} t+\frac{1}{3} \int_{\Omega_{T}} u^{3} \nabla w \cdot \nabla \eta \mathrm{d} x \mathrm{~d} t+\frac{1}{2} \int_{\Omega_{T}} u^{2} \nabla v \cdot \nabla \eta \mathrm{d} x \mathrm{~d} t=0, \\
& \int_{\Omega_{T}}[c \nabla u \cdot \nabla \eta+\phi(u) \eta-w \eta] \mathrm{d} x \mathrm{~d} t=0, \\
& \int_{0}^{T}\left\langle\frac{\partial v}{\partial t}, \eta\right\rangle \mathrm{d} t+\int_{\Omega_{T}}[\rho \nabla v \cdot \nabla \eta+u \lambda(v) \nabla v \cdot \nabla \eta] \mathrm{d} x \mathrm{~d} t+\frac{1}{2} \int_{\Omega_{T}} u^{2} \lambda(v) \nabla w \cdot \nabla \eta \mathrm{d} x \mathrm{~d} t=0 .
\end{aligned}
$$

Proof. Theorem 2.8 and Lemma 2.9 imply that

$$
\max _{t \in[0, T]} \int_{\Omega} \pi^{h}\left[\Phi\left(U_{\varepsilon}^{+}\right)\right](x, t) \mathrm{d} x \leq C .
$$

From the uniform Hölder continuity of $U_{\varepsilon}^{+}$and (3.52), it follows that there exists $\underline{u} \in \mathbb{R}_{>0}$ independent of $h, \tau$, and $\varepsilon$ such that $U_{\varepsilon}^{+}(x, t) \geq \underline{u}>0$ for all $(x, t) \in \bar{\Omega}_{T}$; see, e.g., [23, Corollary 5.3]. Combining this with (3.5a) yields that $u$ is strictly positive.

The rest of the proof is similar to that of Theorem 3.2 with the following minor modifications. We have that (3.36) holds with the extra term $\left(\phi^{+}\left(U_{\varepsilon}^{+}\right)+\phi^{-}\left(U_{\varepsilon}^{-}\right), \zeta^{h}\right)^{h}$ inside the square brackets on the left-hand side. Since $u$ is strictly positive, it is straightforward to show convergence of this extra term to the corresponding term in the weak formulation of the continuous problem.

4. Solution of the nonlinear discrete system. We now discuss algorithms for solving the resulting system of nonlinear equations for $\left\{U_{\varepsilon}^{n}, W_{\varepsilon}^{n}, V_{\varepsilon}^{n}\right\}$ at each time level for the approximations $\left(\mathrm{P}_{\varepsilon}^{h, \tau}\right)$ and $\left(\mathrm{P}_{\delta, \varepsilon}^{h, \tau}\right)$. As (2.11a,b) for $\left(\mathrm{P}_{\varepsilon}^{h, \tau}\right)$ and (2.11a), (2.12) for $\left(\mathrm{P}_{\delta, \varepsilon}^{h, \tau}\right)$ are independent of $V_{\varepsilon}^{n}$, we first solve these to obtain $\left\{U_{\varepsilon}^{n}, W_{\varepsilon}^{n}\right\}$; then we solve $(2.11 \mathrm{c})$ for $V_{\varepsilon}^{n}$. First, we consider $\left(\mathrm{P}_{\varepsilon}^{h, \tau}\right)$. Adapting the techniques in [4, section 3] we introduce $R_{\varepsilon}^{n} \in S^{h}$ by

$$
\left(R_{\varepsilon}^{n}, \chi\right)^{h}=c\left(\nabla U_{\varepsilon}^{n}, \nabla \chi\right)+\left(\phi^{-}\left(U_{\varepsilon}^{n-1}+\varepsilon\right), \chi\right)^{h}-\left(W_{\varepsilon}^{n}, \chi\right)^{h} \quad \forall \chi \in S^{h} .
$$

Hence, for any $\mu \in \mathbb{R}_{>0}$ and on recalling (3.13), $\left(\mathrm{P}_{\varepsilon}^{h, \tau}\right)$ is equivalent to the following.

Given $U_{\varepsilon}^{0} \in K^{h}, V_{\varepsilon}^{0} \in S^{h}$, for $n \geq 1$ find $\left\{U_{\varepsilon}^{n}, W_{\varepsilon}^{n}, V_{\varepsilon}^{n}\right\} \in\left[S^{h}\right]^{3}$ such that (2.11a), (4.1), $R_{\varepsilon}^{n}=\pi^{h}\left[R_{\varepsilon}^{n}-\mu U_{\varepsilon}^{n}\right]_{+}$, and (2.11c) hold. We use this formulation in constructing our iterative method to solve $\left(\mathrm{P}_{\varepsilon}^{h, \tau}\right)$.

Given $\left\{W_{\varepsilon}^{n, 0}, R_{\varepsilon}^{n, 0}\right\} \in\left[S^{h}\right]^{2}$, for $k \geq 1$ find $\left\{U_{\varepsilon}^{n, k}, W_{\varepsilon}^{n, k}, R_{\varepsilon}^{n, k}\right\} \in\left[S^{h}\right]^{3}$ such that for all $\chi \in S^{h}$

$$
\begin{gathered}
\left(\frac{U_{\varepsilon}^{n, k}-U_{\varepsilon}^{n-1}}{\tau_{n}}, \chi\right)^{h}+\frac{b^{n-1}}{3}\left(\nabla W_{\varepsilon}^{n, k}, \nabla \chi\right)=\frac{1}{3}\left(\left(b^{n-1}-\pi^{h}\left[\left(U_{\varepsilon}^{n-1}\right)^{3}\right]\right) \nabla W_{\varepsilon}^{n, k-1}, \nabla \chi\right) \\
-\frac{1}{2}\left(\pi^{h}\left[\left(U_{\varepsilon}^{n-1}\right)^{2}\right] \nabla V_{\varepsilon}^{n-1}, \nabla \chi\right),
\end{gathered}
$$

$c\left(\nabla U_{\varepsilon}^{n, k}, \nabla \chi\right)+\left(\phi^{-}\left(U_{\varepsilon}^{n-1}+\varepsilon\right), \chi\right)^{h}=\left(W_{\varepsilon}^{n, k}+R_{\varepsilon}^{n, k-1}, \chi\right)^{h}$,

where $b^{n-1}:=\left|U_{\varepsilon}^{n-1}\right|_{0, \infty}^{3}$. 
Then, having obtained $\left\{U_{\varepsilon}^{n}, W_{\varepsilon}^{n}\right\}$, we find $V_{\varepsilon}^{n}$ as follows. Given $V_{\varepsilon}^{n, 0} \in S^{h}$, for $k \geq 1$ find $V_{\varepsilon}^{n, k} \in S^{h}$ such that

$$
\begin{aligned}
\left(\frac{V_{\varepsilon}^{n, k}-V_{\varepsilon}^{n-1}}{\tau_{n}}, \chi\right)^{h} & +\rho\left(\nabla V_{\varepsilon}^{n, k}, \nabla \chi\right)+\left(U_{\varepsilon}^{n} \Lambda_{\varepsilon}\left(V_{\varepsilon}^{n, k-1}\right) \nabla V_{\varepsilon}^{n, k}, \nabla \chi\right) \\
= & -\frac{1}{2}\left(\pi^{h}\left[\left(U_{\varepsilon}^{n}\right)^{\frac{1}{2}}\left(U_{\varepsilon}^{n-1}\right)^{\frac{3}{2}}\right] \Lambda_{\varepsilon}\left(V_{\varepsilon}^{n, k-1}\right) \nabla W_{\varepsilon}^{n}, \nabla \chi\right) \quad \forall \chi \in S^{h} .
\end{aligned}
$$

Equation (4.3) is the natural extension of the iterative procedure proposed in [22] for solving a finite element approximation of the thin film equation. As (4.3) is linear, existence of $V_{\varepsilon}^{n, k}$ follows from uniqueness; and this is easily established on noting $\rho \geq 0,(2.5)$, and $U_{\varepsilon}^{n} \in K^{h}$. Hence the iteration (4.3) is well defined.

The algorithm $(4.2 \mathrm{a}-\mathrm{c})$ is a simple adaptation of the algorithm in [4, section 3] for problem $\left(\mathrm{P}_{\varepsilon}^{h, \tau}\right)$ in the absence of the surfactant and van der Waals forces, i.e., $V_{\varepsilon}^{n} \equiv 0$ and $a=0\left(\phi^{-} \equiv 0\right)$.

Defining $A^{n, k-1} \in Z^{h}$ such that

$$
\left(A^{n, k-1}, \chi\right)^{h}:=\frac{1}{3}\left(\pi^{h}\left[\left(U_{\varepsilon}^{n-1}\right)^{3}\right] \nabla W_{\varepsilon}^{n, k-1}, \nabla \chi\right) \quad \forall \chi \in S^{h}
$$

and $X_{\varepsilon}^{n-1} \in S^{h}$ as in (2.30), it follows from (4.2a), (2.22), (4.2b) with $\chi \equiv 1,(1.17)$, and (1.14) that

$$
\begin{aligned}
W_{\varepsilon}^{n, k}=(I-f) W_{\varepsilon}^{n, k-1}-\frac{3}{b^{n-1}} \mathcal{G}^{h}\left[\frac{U_{\varepsilon}^{n, k}-U_{\varepsilon}^{n-1}}{\tau_{n}}\right. & \left.+A^{n, k-1}+X_{\varepsilon}^{n-1}\right] \\
& +f \pi^{h}\left[\phi^{-}\left(U_{\varepsilon}^{n-1}+\varepsilon\right)\right]-f R_{\varepsilon}^{n, k-1} .
\end{aligned}
$$

Therefore, (4.2a,b) may be written equivalently as follows: Find $U_{\varepsilon}^{n, k} \in \bar{S}^{h}\left(U_{\varepsilon}^{n-1}\right):=$ $\left\{\chi \in S^{h}: \chi-U_{\varepsilon}^{n-1} \in Z^{h}\right\}$ such that

$$
\begin{aligned}
& c\left(\nabla U_{\varepsilon}^{n, k}, \nabla \chi\right)+\frac{3}{b^{n-1}}\left(\mathcal{G}^{h}\left[\frac{U_{\varepsilon}^{n, k}-U_{\varepsilon}^{n-1}}{\tau_{n}}\right], \chi\right)^{h} \\
&=\left((I-f)\left(W_{\varepsilon}^{n, k-1}+R_{\varepsilon}^{n, k-1}+\bar{X}_{\varepsilon}^{n, k-1}\right), \chi\right)^{h} \quad \forall \chi \in S^{h},
\end{aligned}
$$

where $\bar{X}_{\varepsilon}^{n, k-1} \in S^{h}$ is such that

$$
\left(\bar{X}_{\varepsilon}^{n, k-1}, \chi\right)^{h}:=-\left(\phi^{-}\left(U_{\varepsilon}^{n-1}+\varepsilon\right)+\frac{3}{b^{n-1}} \mathcal{G}^{h}\left[A^{n, k-1}+X_{\varepsilon}^{n-1}\right], \chi\right)^{h} \quad \forall \chi \in S^{h} .
$$

Existence and uniqueness of $U_{\varepsilon}^{n, k} \in \bar{S}^{h}\left(U_{\varepsilon}^{n-1}\right)$ satisfying (4.6) then follows since, on noting (2.22), this is the Euler-Lagrange equation of the convex minimization problem

$$
\min _{\chi \in \bar{S}^{h}\left(U_{\varepsilon}^{n-1}\right)}\left\{\frac{c}{2}|\chi|_{1}^{2}+\frac{3}{2 b^{n-1} \tau_{n}}\left|\nabla \mathcal{G}^{h}\left(\chi-U_{\varepsilon}^{n-1}\right)\right|_{0}^{2}-\left(W_{\varepsilon}^{n, k-1}+R_{\varepsilon}^{n, k-1}+\bar{X}_{\varepsilon}^{n, k-1}, \chi\right)^{h}\right\} .
$$

Finally, $W_{\varepsilon}^{n, k}$ and $R_{\varepsilon}^{n, k}$ are uniquely defined by (4.5) and (4.2c), respectively. Hence the iterative procedure $(4.2 \mathrm{a}-\mathrm{c})$ is well defined for any $\mu>0$.

THEOREM 4.1. Let the assumptions (A) hold. Then there exists a $\mu_{0}$ such that for all $\mu \in\left(0, \mu_{0}\right)$ and $\left\{W_{\varepsilon}^{n, 0}, R_{\varepsilon}^{n, 0}\right\} \in\left[S^{h}\right]^{2}$ the sequence $\left\{U_{\varepsilon}^{n, k}, W_{\varepsilon}^{n, k}\right\}_{k \geq 0}$ generated by the algorithm $(4.2 \mathrm{a}-\mathrm{c})$ satisfies

$$
U_{\varepsilon}^{n, k} \rightarrow U_{\varepsilon}^{n} \quad \text { and } \quad \int_{\Omega} \pi^{h}\left[\left(U_{\varepsilon}^{n-1}\right)^{3}\right]\left|\nabla\left(W_{\varepsilon}^{n}-W_{\varepsilon}^{n, k}\right)\right|^{2} \mathrm{~d} x \rightarrow 0 \quad \text { as } k \rightarrow \infty .
$$


Proof. This is a simple adaptation of the proof of Theorem 3.1 in [4]. On letting

$$
E^{k}:=U_{\varepsilon}^{n}-U_{\varepsilon}^{n, k} \in Z^{h}, \quad F^{k}:=W_{\varepsilon}^{n}-W_{\varepsilon}^{n, k} \in S^{h}, \quad \text { and } \quad D^{k}:=R_{\varepsilon}^{n}-R_{\varepsilon}^{n, k} \in S^{h},
$$

it is an easy exercise to show that

$$
\begin{aligned}
& \left|D^{k}\right|_{h}^{2}+\frac{\mu \tau_{n}}{3}\left|\left[b^{n-1}-\pi^{h}\left[\left(U_{\varepsilon}^{n-1}\right)^{3}\right]\right]^{\frac{1}{2}} \nabla F^{k}\right|_{0}^{2}+\frac{2}{3} \mu \tau_{n}\left|\left[\pi^{h}\left[\left(U_{\varepsilon}^{n-1}\right)^{3}\right]\right]^{\frac{1}{2}} \nabla F^{k}\right|_{0}^{2} \\
& \quad+\left(2 \mu c-C \mu^{2}\right)\left|E^{k}\right|_{1}^{2} \leq\left|D^{k-1}\right|_{h}^{2}+\frac{\mu \tau_{n}}{3}\left|\left[b^{n-1}-\pi^{h}\left[\left(U_{\varepsilon}^{n-1}\right)^{3}\right]\right]^{\frac{1}{2}} \nabla F^{k-1}\right|_{0}^{2} .
\end{aligned}
$$

Now (4.9) yields that $\left\{\left|D^{k}\right|_{h}^{2}+\frac{\mu \tau_{n}}{3}\left|\left[b^{n-1}-\pi^{h}\left[\left(U_{\varepsilon}^{n-1}\right)^{3}\right]\right]^{\frac{1}{2}} \nabla F^{k}\right|_{0}^{2}\right\}_{k \geq 0}$ is a decreasing sequence for $\mu$ sufficiently small and hence has a limit. Therefore, the desired results (4.8) follow from this and (4.9).

Remark 4.2. The linear system (4.3) can be solved efficiently using a conjugate gradient algorithm. Although we are unable to show convergence of the iteration (4.3) for $V_{\varepsilon}^{n}$, we observed good convergence properties in practice.

4.1. Inclusion of repulsive van der Waals forces. We now consider an algorithm for solving the nonlinear algebraic system at each time level in $\left(\mathrm{P}_{\delta, \varepsilon}^{h, \tau}\right)$. Our method for $\left\{U_{\varepsilon}^{n}, W_{\varepsilon}^{n}\right\}$ satisfying (2.11a) and (2.12) is based on the general splitting algorithm of [25]; see also $[2,5]$, where this algorithm has been adapted to solve similar variational inequality problems arising from Cahn-Hilliard systems. $V_{\varepsilon}^{n}$ satisfying (2.11c) is solved as before using (4.3). We now introduce our algorithm for $\left\{U_{\varepsilon}^{n}, W_{\varepsilon}^{n}\right\}$. Let $\mathcal{B}_{n}: S^{h} \rightarrow S^{h}$ be such that for all $q^{h} \in S^{h}, \chi \in S^{h}$

$$
\left(\mathcal{B}_{n}\left(q^{h}\right), \chi\right)^{h}:=c\left(\nabla q^{h}, \nabla \chi\right)+\left(\phi^{-}\left(U_{\varepsilon}^{n-1}\right), \chi\right)^{h} .
$$

Hence (2.12) can be rewritten as

$$
\left(\mathcal{B}_{n}\left(U_{\varepsilon}^{n}\right)+\phi^{+}\left(U_{\varepsilon}^{n}\right), \chi\right)^{h}=\left(W_{\varepsilon}^{n}, \chi\right)^{h} \quad \forall \chi \in S^{h} .
$$

Now, for $n$ fixed, multiplying (4.10) with $\mu \in \mathbb{R}_{>0}$, adding $\left(U_{\varepsilon}^{n}, \chi\right)^{h}$ to both sides, rearranging on noting (2.11a), and defining $X_{\varepsilon}^{n-1} \in S^{h}$ as in (2.30), it follows that $\left\{U_{\varepsilon}^{n}, W_{\varepsilon}^{n}\right\} \in\left[S^{h}\right]^{2}$ solving (2.11a) and (2.12) satisfy for all $\chi \in S^{h}$

$$
\begin{aligned}
\left(\frac{U_{\varepsilon}^{n}-U_{\varepsilon}^{n-1}}{\tau_{n}}, \chi\right)^{h}+\frac{b^{n-1}}{3}\left(\nabla W_{\varepsilon}^{n}, \nabla \chi\right) & =\frac{1}{3}\left(\left(b^{n-1}-\pi^{h}\left[\left(U_{\varepsilon}^{n-1}\right)^{3}\right]\right) \nabla W_{\varepsilon}^{n}, \nabla \chi\right) \\
\text { 1a) } & -\left(X_{\varepsilon}^{n-1}, \chi\right)^{h}, \\
\text { 1b) } &
\end{aligned}
$$

where $Y_{\varepsilon}^{n} \in S^{h}$ is such that

$$
\left(Y_{\varepsilon}^{n}, \chi\right)^{h}:=\left(U_{\varepsilon}^{n}, \chi\right)^{h}-\mu\left(\mathcal{B}_{n}\left(U_{\varepsilon}^{n}\right)-W_{\varepsilon}^{n}, \chi\right)^{h} \quad \forall \chi \in S^{h} .
$$

For later use we introduce also $\bar{Y}_{\varepsilon}^{n} \in S^{h}$ such that

$$
\left(\bar{Y}_{\varepsilon}^{n}, \chi\right)^{h}:=\left(U_{\varepsilon}^{n}, \chi\right)^{h}+\mu\left(\mathcal{B}_{n}\left(U_{\varepsilon}^{n}\right)-W_{\varepsilon}^{n}, \chi\right)^{h} \quad \forall \chi \in S^{h}
$$

and note that $\bar{Y}_{\varepsilon}^{n}=2 U_{\varepsilon}^{n}-Y_{\varepsilon}^{n}$. We use this as a basis for constructing our iterative procedure to find $\left\{U_{\varepsilon}^{n}, W_{\varepsilon}^{n}\right\} \in\left[S^{h}\right]^{2}$ satisfying (4.11a,b).

Given $\left\{U_{\varepsilon}^{n, k-1}, W_{\varepsilon}^{n, k-1}\right\} \in\left[S^{h}\right]^{2}$ for $k \geq 1$, we define $Y_{\varepsilon}^{n, k-1} \in S^{h}$ such that

$$
\left(Y_{\varepsilon}^{n, k-1}, \chi\right)^{h}:=\left(U_{\varepsilon}^{n, k-1}, \chi\right)^{h}-\mu\left(\mathcal{B}_{n}\left(U_{\varepsilon}^{n, k-1}\right)-W_{\varepsilon}^{n, k-1}, \chi\right)^{h} \quad \forall \chi \in S^{h} .
$$


Then we find $U_{\varepsilon}^{n, k-\frac{1}{2}} \in S^{h}$ such that

$$
\left(U_{\varepsilon}^{n, k-\frac{1}{2}}+\mu \phi^{+}\left(U_{\varepsilon}^{n, k-\frac{1}{2}}\right), \chi\right)^{h}=\left(Y_{\varepsilon}^{n, k-1}, \chi\right)^{h} \quad \forall \chi \in S^{h}
$$

and find $\left\{U_{\varepsilon}^{n, k}, W_{\varepsilon}^{n, k}\right\} \in\left[S^{h}\right]^{2}$ such that for all $\chi \in S^{h}$

$$
\begin{aligned}
&\left(\frac{U_{\varepsilon}^{n, k}-U_{\varepsilon}^{n-1}}{\tau_{n}}, \chi\right)^{h}+ \frac{b^{n-1}}{3}\left(\nabla W_{\varepsilon}^{n, k}, \nabla \chi\right) \\
&=\frac{1}{3}\left(\left(b^{n-1}-\pi^{h}\left[\left(U_{\varepsilon}^{n-1}\right)^{3}\right]\right) \nabla W_{\varepsilon}^{n, k-1}, \nabla \chi\right)-\left(X_{\varepsilon}^{n-1}, \chi\right)^{h} \\
&\left(U_{\varepsilon}^{n, k}, \chi\right)^{h}+\mu\left(\mathcal{B}_{n}\left(U_{\varepsilon}^{n, k}\right)-W_{\varepsilon}^{n, k}, \chi\right)^{h}=\left(\bar{Y}_{\varepsilon}^{n, k}, \chi\right)^{h}
\end{aligned}
$$

where $\bar{Y}_{\varepsilon}^{n, k}:=2 U_{\varepsilon}^{n, k-\frac{1}{2}}-Y_{\varepsilon}^{n, k-1}$. Existence and uniqueness of $U_{\varepsilon}^{n, k-\frac{1}{2}}>0$ in (4.12b) follow from the monotonicity of $\varphi: \mathbb{R}_{>0} \rightarrow \mathbb{R}$, where $\varphi(s):=s+\mu \phi^{+}(s)$, and the fact that $\lim _{s \rightarrow \infty} \varphi(s)=-\lim _{s \backslash 0} \varphi(s)=\infty$.

It remains to show that $(4.12 \mathrm{c}, \mathrm{d})$ possess a unique solution $\left\{U_{\varepsilon}^{n, k}, W_{\varepsilon}^{n, k}\right\} \in\left[S^{h}\right]^{2}$. This is a simple adaptation of the existence and uniqueness proof for $\left\{U_{\varepsilon}^{n, k}, W_{\varepsilon}^{n, k}\right.$, $\left.R_{\varepsilon}^{n, k}\right\}$ in (4.2a-c). Similarly to (4.5), it follows from (4.12c), (2.22), (4.12d) with $\chi \equiv 1,(1.17)$, and (1.14) that

$$
\begin{aligned}
W_{\varepsilon}^{n, k}=(I-f) W_{\varepsilon}^{n, k-1}-\frac{3}{b^{n-1}} \mathcal{G}^{h} & {\left[\frac{U_{\varepsilon}^{n, k}-U_{\varepsilon}^{n-1}}{\tau_{n}}+A^{n, k-1}+X_{\varepsilon}^{n-1}\right] } \\
& +f \pi^{h}\left[\phi^{-}\left(U_{\varepsilon}^{n-1}\right)\right]+\mu^{-1} f\left(U_{\varepsilon}^{n, k}-\bar{Y}_{\varepsilon}^{n, k}\right),
\end{aligned}
$$

where $A^{n, k-1} \in Z^{h}$ is defined as in (4.4). Then similarly to (4.6), (4.12c,d) may be written equivalently as follows: Find $U_{\varepsilon}^{n, k} \in \bar{S}^{h}\left(U_{\varepsilon}^{n-1}\right) \equiv S^{h}\left(U_{\varepsilon}^{n-1}\right)$ such that for all $\chi \in S^{h}$

$$
\begin{aligned}
& \left(U_{\varepsilon}^{n, k},(I-f) \chi\right)^{h}+\mu\left[c\left(\nabla U_{\varepsilon}^{n, k}, \nabla \chi\right)+\frac{3}{b^{n-1}}\left(\mathcal{G}^{h}\left[\frac{U_{\varepsilon}^{n, k}-U_{\varepsilon}^{n-1}}{\tau_{n}}\right], \chi\right)^{h}\right] \\
& =\left(\bar{Y}_{\varepsilon}^{n, k}+\mu\left[W_{\varepsilon}^{n, k-1}-\phi^{-}\left(U_{\varepsilon}^{n-1}\right)-\frac{3}{b^{n-1}} \mathcal{G}^{h}\left[A^{n, k-1}+X_{\varepsilon}^{n-1}\right]\right],(I-f) \chi\right)^{h} .
\end{aligned}
$$

Similarly to (4.7), existence and uniqueness of $U_{\varepsilon}^{n, k} \in \bar{S}^{h}\left(U_{\varepsilon}^{n-1}\right)$ satisfying (4.14) then follow since this is the Euler-Lagrange equation of the convex minimization problem

$$
\begin{aligned}
\min _{\chi \in \bar{S}^{h}\left(U_{\varepsilon}^{n-1}\right)}\{ & \frac{1}{2}|\chi|_{h}^{2}+\mu\left[\frac{c}{2}|\chi|_{1}^{2}+\frac{3}{2 b^{n-1} \tau_{n}}\left|\nabla \mathcal{G}^{h}\left(\chi-U_{\varepsilon}^{n-1}\right)\right|_{0}^{2}\right] \\
& \left.-\left(\bar{Y}_{\varepsilon}^{n, k}+\mu\left[W_{\varepsilon}^{n, k-1}-\phi^{-}\left(U_{\varepsilon}^{n-1}\right)-\frac{3}{b^{n-1}} \mathcal{G}^{h}\left[A^{n, k-1}+X_{\varepsilon}^{n-1}\right]\right], \chi\right)^{h}\right\} .
\end{aligned}
$$

Finally, $W_{\varepsilon}^{n, k}$ is uniquely defined by (4.13). Hence the iterative procedure (4.12a-d) is well defined for any $\mu>0$.

THEOREM 4.3. Let the assumptions (A) hold. Then for all $\mu \in \mathbb{R}_{>0}$ and $\left\{U_{\varepsilon}^{n, 0}, W_{\varepsilon}^{n, 0}\right\} \in\left[S^{h}\right]^{2}$ the sequence $\left\{U_{\varepsilon}^{n, k}, W_{\varepsilon}^{n, k}\right\}_{k \geq 0}$ generated by the algorithm (4.12a-d) satisfies

$$
U_{\varepsilon}^{n, k} \rightarrow U_{\varepsilon}^{n} \quad \text { and } \quad \int_{\Omega} \pi^{h}\left[\left(U_{\varepsilon}^{n-1}\right)^{3}\right]\left|\nabla\left(W_{\varepsilon}^{n}-W_{\varepsilon}^{n, k}\right)\right|^{2} \mathrm{~d} x \rightarrow 0 \quad \text { as } k \rightarrow \infty .
$$

In addition, it holds that $U_{\varepsilon}^{n, k-\frac{1}{2}} \rightarrow U_{\varepsilon}^{n}$ as $k \rightarrow \infty$. 
Proof. A simple adaptation of the proof of Theorem 3.1 in [5] yields that

$$
\begin{aligned}
& c\left|U_{\varepsilon}^{n}-U_{\varepsilon}^{n, k}\right|_{1}^{2}+\frac{1}{4 \mu}\left|Y_{\varepsilon}^{n}-Y_{\varepsilon}^{n, k}\right|_{h}^{2}+\frac{\tau_{n}}{6}\left|\left[b^{n-1}-\pi^{h}\left[\left(U_{\varepsilon}^{n-1}\right)^{3}\right]\right]^{\frac{1}{2}} \nabla\left(W_{\varepsilon}^{n}-W_{\varepsilon}^{n, k}\right)\right|_{0}^{2} \\
& +\frac{\tau_{n}}{3}\left|\left[\pi^{h}\left[\left(U_{\varepsilon}^{n-1}\right)^{3}\right]\right]^{\frac{1}{2}} \nabla\left(W_{\varepsilon}^{n}-W_{\varepsilon}^{n, k}\right)\right|_{0}^{2}+\left(\phi^{+}\left(U_{\varepsilon}^{n}\right)-\phi^{+}\left(U_{\varepsilon}^{n, k-\frac{1}{2}}\right), U_{\varepsilon}^{n}-U_{\varepsilon}^{n, k-\frac{1}{2}}\right)^{h}
\end{aligned}
$$

$$
\leq \frac{1}{4 \mu}\left|Y_{\varepsilon}^{n}-Y_{\varepsilon}^{n, k-1}\right|_{h}^{2}+\frac{\tau_{n}}{6}\left|\left[b^{n-1}-\pi^{h}\left[\left(U_{\varepsilon}^{n-1}\right)^{3}\right]\right]^{\frac{1}{2}} \nabla\left(W_{\varepsilon}^{n}-W_{\varepsilon}^{n, k-1}\right)\right|_{0}^{2} .
$$

Therefore, on noting the monotonicity of $\phi^{+}$, we have that $\left\{\frac{1}{4 \mu}\left|Y_{\varepsilon}^{n}-Y_{\varepsilon}^{n, k}\right|_{h}^{2}+\right.$ $\left.\frac{\tau_{n}}{6}\left|\left[b^{n-1}-\pi^{h}\left[\left(U_{\varepsilon}^{n-1}\right)^{3}\right]\right]^{\frac{1}{2}} \nabla\left(W_{\varepsilon}^{n}-W_{\varepsilon}^{n, k}\right)\right|_{0}^{2}\right\}_{k>0}$ is a decreasing sequence for all $\mu>0$. Since it is bounded below, the sequence has a limit. Hence the desired results follow from this and (4.16).

Remark 4.4. Note that the algorithm (4.12a-d) can easily be modified to solve the variational inequality that arises at each time step in $\left(\mathrm{P}_{\varepsilon}^{h, \tau}\right)$. In particular, let $\overline{\mathcal{B}}_{n}: S^{h} \rightarrow S^{h}$ be such that $\left(\overline{\mathcal{B}}_{n}\left(q^{h}\right), \chi\right)^{h}:=c\left(\nabla q^{h}, \nabla \chi\right)+\left(\phi^{-}\left(U_{\varepsilon}^{n-1}+\varepsilon\right), \chi\right)^{h}$ for all $q^{h} \in S^{h}, \chi \in S^{h}$, substitute $\overline{\mathcal{B}}_{n}$ for $\mathcal{B}_{n}$ in $(4.12 \mathrm{a}-\mathrm{d})$, and replace (4.12b) with the following: Find $U_{\varepsilon}^{n, k-\frac{1}{2}} \in K^{h}$ such that $\left(U_{\varepsilon}^{n, k-\frac{1}{2}}-Y_{\varepsilon}^{n, k}, \eta-U_{\varepsilon}^{n, k-\frac{1}{2}}\right)^{h} \geq 0$ for all $\eta \in K^{h}$. Then this new procedure satisfies the statement of Theorem 4.3 as well; see [5, section 3] for a similar proof. However, we employed algorithm (4.2a-c) to solve $\left(\mathrm{P}_{\varepsilon}^{h, \tau}\right)$ since in practice it exhibited superior convergence properties.

Remark 4.5. We see from (4.6) for $\left(\mathrm{P}_{\varepsilon}^{h, \tau}\right)$ and (4.14) for $\left(\mathrm{P}_{\delta, \varepsilon}^{h, \tau}\right)$ that at each iteration for $U_{\varepsilon}^{n}$ one needs to solve only a fixed linear system with constant coefficients. On a uniform mesh this can be done efficiently using a discrete cosine transform; see $[12$, section 5], where a similar problem is solved.

5. Numerical results. First, we present numerical experiments in one space dimension in the absence of van der Waals forces, $a=\delta=0(\phi \equiv 0)$. Throughout we chose a uniform partitioning of $\Omega=(-L, L)$, where $L \geq 1$, with mesh points $p_{j}=-L+(j-1) h, j=1 \rightarrow \# J$, where $h=\frac{2 L}{\# J-1}$. In addition, we chose uniform time steps $\tau_{n}=\tau=1.28 \times 10^{-2} h$ and set the regularization parameter $\varepsilon=1.28 \times 10^{-3} h$. For the initial profiles $u^{0}(x)$ and $v^{0}(x)$, we chose either

$$
\begin{aligned}
& \text { (i) } \quad u^{0}(x)=\left[\frac{1}{4}-x^{2}\right]_{+} \quad \text { or } \quad \text { (ii) } \quad u^{0}(x)=1 \\
& \text { with } \quad v^{0}(x)=\frac{v_{\max }^{0}}{2}\left[(1-\gamma)-\tanh \left(A\left(|x|-x_{0}\right)\right)\right]_{+},
\end{aligned}
$$

where $v_{\max }^{0} \geq 0, \gamma \in[0,1), A>0$, and $x_{0} \in(0, L)$. (i) with $v_{\max }^{0}>0$, (i) with $v_{\max }^{0}=0$, and (ii) with $v_{\max }^{0}>0$ resemble a liquid drop on a plain surface with and without surfactant on top of it and a uniform liquid film with surfactant, respectively. Note that for $\gamma>0$ the surfactant $v^{0}$ has compact support $[-l, l]$, where $l=x_{0}+$ $A^{-1} \tanh ^{-1}(1-\gamma)$. Throughout we chose $U_{\varepsilon}^{0} \equiv \pi^{h} u^{0}$ and $V_{\varepsilon}^{0} \equiv \pi^{h} v^{0}$ as the discrete initial data for $\left(\mathrm{P}_{\varepsilon}^{h, \tau}\right)$ and $\left(\mathrm{P}_{\delta, \varepsilon}^{h, \tau}\right)$.

For the iterative algorithms $(4.2 \mathrm{a}-\mathrm{c}),(4.12 \mathrm{a}-\mathrm{d})$, and (4.3), we set, for $n \geq 1$, $Z^{n, 0} \equiv Z^{n-1}$ for $Z=U_{\varepsilon}, W_{\varepsilon}, V_{\varepsilon}$, and $R_{\varepsilon}$, where

$$
R_{\varepsilon}^{0}=0 \quad \text { and } \quad\left(W_{\varepsilon}^{0}, \chi\right)^{h}=c\left(\nabla U_{\varepsilon}^{0}, \nabla \chi\right)+\left(\phi\left(U_{\varepsilon}^{0}\right), \chi\right)^{h} \quad \forall \chi \in S^{h},
$$

and for each $n$ adopted the stopping criteria

$$
\left|U_{\varepsilon}^{n, k}-U_{\varepsilon}^{n, k-1}\right|_{0, \infty}<t o l \text { and }\left|V_{\varepsilon}^{n, k}-V_{\varepsilon}^{n, k-1}\right|_{0, \infty}<t o l,
$$


TABLE 1

(i) with $v_{\max }^{0}=0$, source-type solution errors.

\begin{tabular}{|r||cccccc|}
\hline$\# J$ & 65 & 129 & 257 & 513 & 1025 & 2049 \\
\hline $\max _{n=1 \rightarrow N}\left\|\pi^{h} u\left(\cdot, t_{n}\right)-U_{\varepsilon}^{n}(\cdot)\right\|_{0, \infty} \times 10^{4}$ & 74.68 & 62.90 & 42.40 & 8.263 & 2.538 & 0.900 \\
\hline $\max _{n=1 \rightarrow N}\left\|\pi^{h} v\left(\cdot, t_{n}\right)-V_{\varepsilon}^{n}(\cdot)\right\|_{0, \infty} \times 10^{5}$ & 1.549 & 1.391 & 2.041 & 2.944 & 3.499 & 3.989 \\
\hline
\end{tabular}

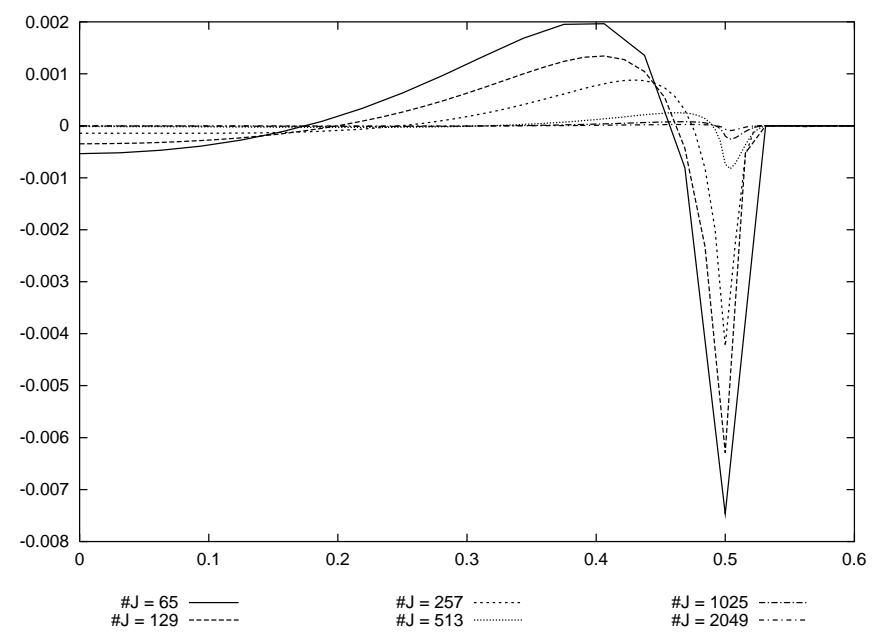

FIG. 1. $\pi^{h} u^{0}(x)-U_{\varepsilon}(x, T)$ plotted against $x$ for $T=4$ with $v^{0} \equiv 0$.

respectively, with tol $=10^{-8}$. Furthermore, we chose $\mu=\frac{1}{1.13 h}$ and set $\left\{U_{\varepsilon}^{n}, W_{\varepsilon}^{n}, R_{\varepsilon}^{n}\right\}$ $\equiv\left\{\pi^{h}\left[U_{\varepsilon}^{n, k}\right]_{+}, W_{\varepsilon}^{n, k}, R_{\varepsilon}^{n, k}\right\}$ for $(4.2 \mathrm{a}-\mathrm{c})$, while we used $\mu=0.625 h$ and set $\left\{U_{\varepsilon}^{n}, W_{\varepsilon}^{n}\right\}$ $\equiv\left\{U_{\varepsilon}^{n, k}, W_{\varepsilon}^{n, k}\right\}$ if $U_{\varepsilon}^{n, k}>0$ and $\left\{U_{\varepsilon}^{n}, W_{\varepsilon}^{n}\right\} \equiv\left\{U_{\varepsilon}^{n, k-\frac{1}{2}}, W_{\varepsilon}^{n, k}\right\}$ otherwise for (4.12a-d). For the iteration (4.3) we set $V_{\varepsilon}^{n} \equiv V_{\varepsilon}^{n, k}$.

In our first set of experiments we set the parameters $L=1, c=2 \times 10^{-2}, \rho=0$, and we chose the initial data (i) with $v_{\max }^{0}=0$ and a final time $T=4$. We note from the weak formulation $(3.25 \mathrm{a}-\mathrm{c})$ that this initial data is a steady state for $(\mathrm{P})$; that is, $u(x, t)=u^{0}(x), v(x, t)=0$ for all $(x, t) \in \Omega_{T}$. The results for various choices of $h$ are displayed in Table 1, where all values are correct to four significant figures.

Remark 5.1. In order to obtain a discretization that leads to a discrete analogue of the energy estimate (1.5) we needed to approximate $\lambda(v)$ in a subtle way; see (2.7). In particular, the resulting scheme does not guarantee that $V_{\varepsilon} \equiv 0$ if the initial data have this property. However, the results in Table 1 show that the error between $V_{\varepsilon}$ and $v$ is small.

In Figure 1 we plot $\pi^{h} u^{0}(x)-U_{\varepsilon}(x, T)$ for $\# J=2^{k}+1, k=6 \rightarrow 11$, on a short interval about the initial free boundary point on the right-hand side, $x=0.5$. For each $\# J$ there are a few points outside the support of $u^{0}(x)$ which are much larger than tol $=10^{-8}$. Outside the region plotted $\left|\pi^{h} u^{0}(x)-U_{\varepsilon}(x, T)\right|$ is "zero," i.e., much smaller than tol. This behavior compares with results in [4], where similar errors can be observed. Note that the different choice of $c$ here acts as a time scaling factor.

We see from Figure 2 that for $\# J=2^{10}+1$ the initial profile $U_{\varepsilon}^{0} \equiv \pi^{h} u^{0}$ is graphically preserved in the absence of surfactant. This is underlined by the fact that the energy

$$
E(t):=\frac{t-t_{n-1}}{\tau_{n}} \mathcal{E}\left(U_{\varepsilon}^{n}, V_{\varepsilon}^{n}\right)+\frac{t_{n}-t}{\tau_{n}} \mathcal{E}\left(U_{\varepsilon}^{n-1}, V_{\varepsilon}^{n-1}\right), \quad t \in\left[t_{n-1}, t_{n}\right], \quad n \geq 1,
$$



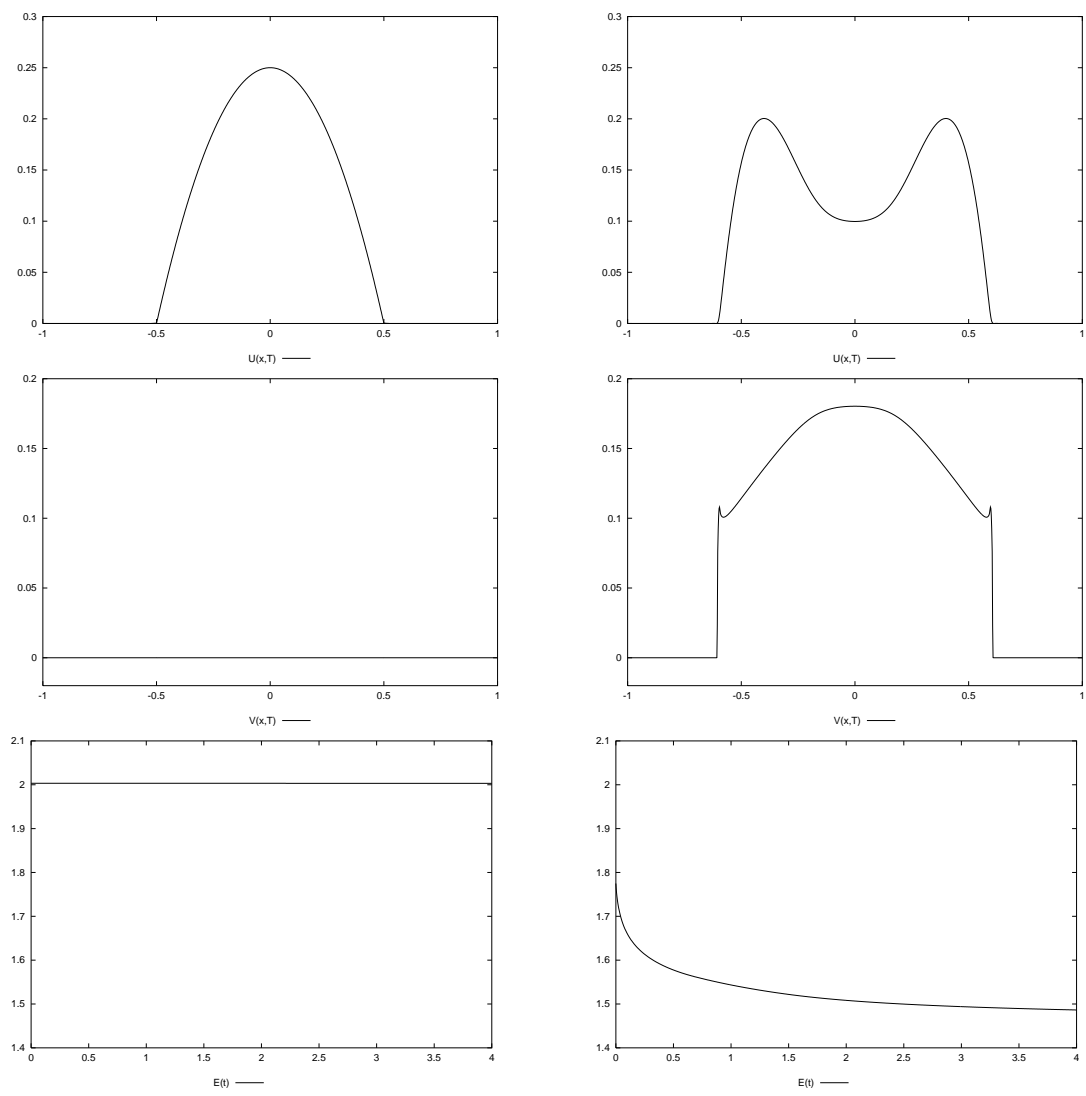

(a)

(b)

FIG. 2. Comparison of $U_{\varepsilon}(x, T), V_{\varepsilon}(x, T)$ at $T=4$ and $E(t)$ for $t \in[0, T]$ in the (a) absence and (b) presence of surfactant, respectively.

remains constant over the whole time period $[0, T]$. When surfactant is included, however, this has a dramatic effect on the shape of the solution $U_{\varepsilon}(x, T)$. For the plot in Figure 2, we took $v^{0}$ as described in (5.1b) with $A=50, x_{0}=0.1, \gamma=10^{-4}$, and $v_{\max }^{0}=0.9$ so that the support of $v^{0} \subset(-0.2,0.2) \subset[-0.5,0.5]$, the support of $u^{0}$. Eventually the solutions $U_{\varepsilon}$ and $V_{\varepsilon}$ reach a "numerical steady state"; i.e., we obtain that $\left\{U_{\varepsilon}^{n}, W_{\varepsilon}^{n}, V_{\varepsilon}^{n}\right\} \equiv\left\{U_{\varepsilon}^{n, 1}, W_{\varepsilon}^{n, 1}, V_{\varepsilon}^{n, 1}\right\}$ for $n$ sufficiently large for the stated stopping criteria on $U_{\varepsilon}$ and $V_{\varepsilon}$ (see (5.2)). For the parameters mentioned above and a stopping tolerance of $t o l=10^{-10}$ this state is reached at $T=3413$. In Figure 3 we plot $U_{\varepsilon}(\cdot, t)$ and $V_{\varepsilon}(\cdot, t)$ for $t=0, t=50, t=200$, and $t=3413$, respectively.

Remark 5.2. In the case $\rho=0$, the only mechanism for surfactant spreading is transport via the fluid velocity. If the support of the initial data of the surfactant is contained in the set of points initially wetted, then one can show that the support of the surfactant at time $t$ is contained in the set $\mathcal{W}(t):=\{x: u(x, \tau)>0$ for some $\tau \in$ $[0, t]\}$, which is the set of points which have been wetted at some time in the past. For the discrete problem a similar property follows directly from (2.11c), since $V_{\varepsilon}^{n}\left(p_{j}\right)$ can only be nonzero if either $V_{\varepsilon}^{n-1}\left(p_{j}\right) \neq 0$ or $\left(U_{\varepsilon}^{n}, \chi_{j}\right) \neq 0$. Therefore, the only modification is that the support of the surfactant can be one mesh point ahead of the discrete analogue of $\mathcal{W}(t)$. 

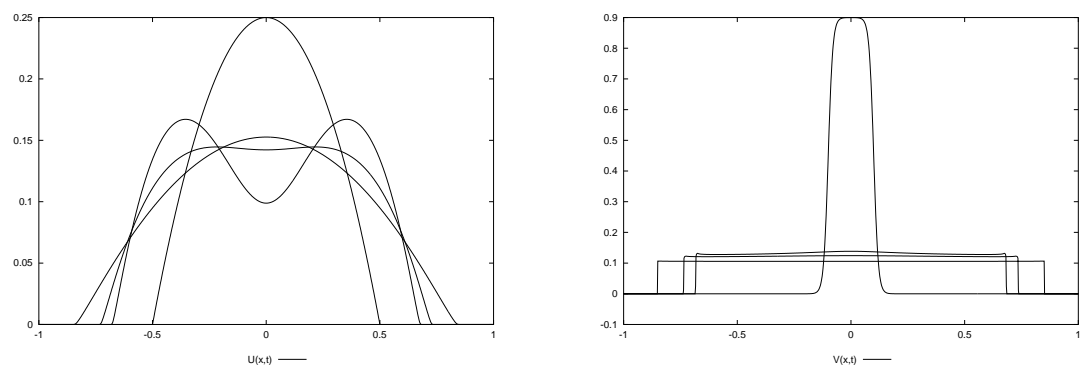

FiG. 3. $U_{\varepsilon}(x, t)$ and $V_{\varepsilon}(x, t)$ for $t=0,50,200,3413$.
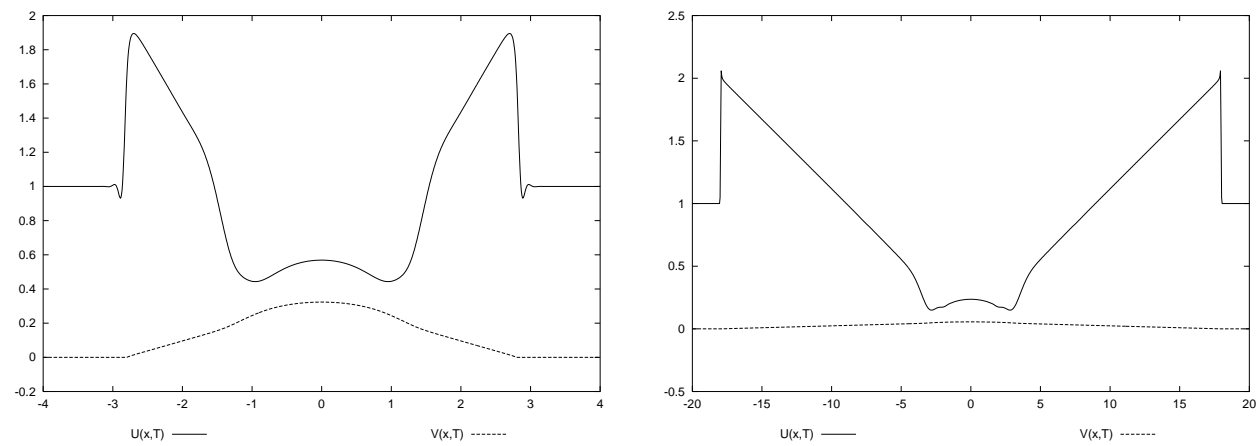

FIG. 4. $U_{\varepsilon}(x, T)$ and $V_{\varepsilon}(x, T)$ plotted against $x$ for $T=4$ (left) and $T=1000$ (right).

Remark 5.3. A parabolic profile for the drop together with a constant surfactant density on the support of the drop is a steady state for the system $(1.1 \mathrm{a}-\mathrm{c})$ if $\rho=0$. It is the discrete analogue of such a steady state that we observe for large times in Figure 3 .

Remark 5.4. For the thin film equation (in the no-slip case) it is conjectured that the support of the film does not increase. In the case that a surfactant is placed on the film this property does not seem to be true any longer.

In addition, we performed experiments for a uniform liquid layer, i.e., $u^{0} \equiv 1$. We chose the parameters similar to the ones reported in [26]. In particular, we took $L=4, T=4, c=10^{-5}, \rho=2 \times 10^{-4}, v_{\max }^{0}=1, \gamma=0, A=10, x_{0}=0.5$, and $\# J=2^{10}+1$. The computed solutions $U_{\varepsilon}(x, T)$ and $V_{\varepsilon}(x, T)$ can be seen on the left-hand side of Figure 4.

We note that $U_{\varepsilon}(x, T)$ and $V_{\varepsilon}(x, T)$ approach similarity solutions of $(\mathrm{P})$ for the case $\rho=c=a=\delta=0$; see [24]. This can be seen on the right-hand side of Figure 4, where we plot the two functions for the values $L=20, T=1000, c=10^{-8}, \rho=0$, $\gamma=0, v_{\max }^{0}=1, A=10, x_{0}=0.5$, and $\# J=2^{10}+1$. We recall that $\left(\mathrm{P}_{\varepsilon}^{h, \tau}\right)$ is only well-posed for $c>0$. In order to formulate the similarity solutions, we make use of the following transformation of coordinates. Let $\xi:=(1+t)^{-\frac{1}{3}} x, \bar{u}(\xi, t):=U_{\varepsilon}(x, t)$ and $\bar{v}(\xi, t):=(1+t)^{\frac{1}{3}} V_{\varepsilon}(x, t)$. Then the similarity solutions for $\bar{u}$ and $\bar{v}$ are given by

$$
\bar{u}_{0}(\xi)=\left\{\begin{array}{ll}
\frac{2 \xi}{\xi_{s}}, & 0 \leq \xi<\xi_{s}, \\
1, & \xi_{s} \leq \xi,
\end{array} \quad \text { and } \quad \bar{v}_{0}(\xi)= \begin{cases}\frac{\xi_{s}}{6}\left(\xi_{s}-\xi\right), & 0 \leq \xi<\xi_{s} \\
0, & \xi_{s} \leq \xi\end{cases}\right.
$$

where $\xi_{s}:=\left(12 \int_{0}^{L} v^{0}(x) \mathrm{d} x\right)^{\frac{1}{3}}$ is the position of the shock. The corresponding plot is shown on the left-hand side of Figure 5. 

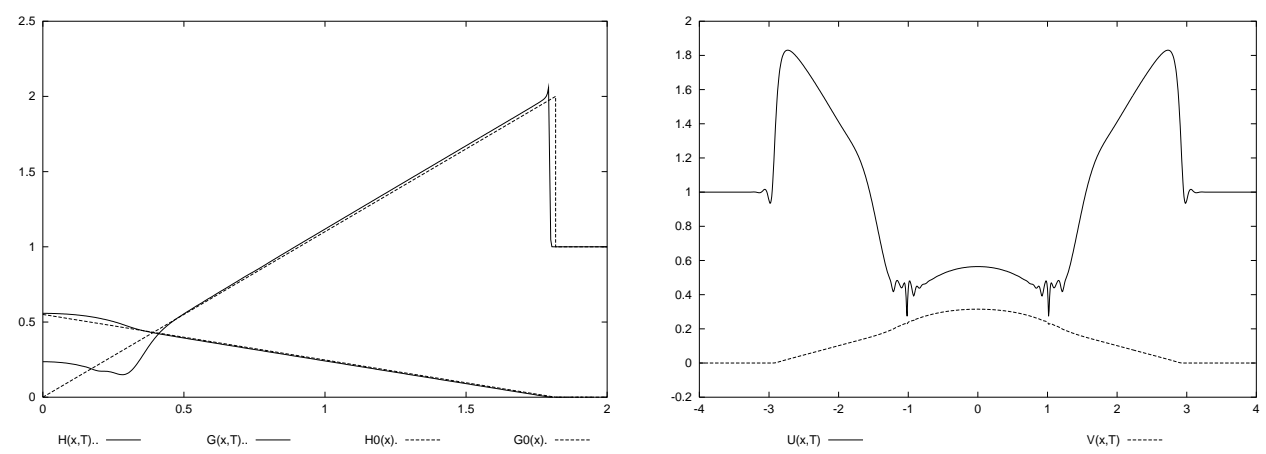

FIG. 5. $\bar{u}(\xi, T), \bar{v}(\xi, T)$ with the corresponding similarity solutions $\bar{u}_{0}(\xi), \bar{v}_{0}(\xi)$ plotted against $\xi$ for $T=1000$ (left) and $U_{\varepsilon}(x, T), V_{\varepsilon}(x, T)$ plotted against $x$ for $T=4.33$ when $\phi \not \equiv 0$ (right).

5.1. Inclusion of van der Waals forces. In addition, we conducted numerical experiments in which we considered the effect of both attractive and repulsive van der Waals forces being present. Note that this corresponds to $a>0$ and $\delta>0$, respectively.

We note that $(4.12 \mathrm{~b})$ is a decoupled system of $\# J$ equations and that for $\chi \equiv \chi_{j}$ one has to find $s=U_{\varepsilon}^{n, k-\frac{1}{2}}\left(p_{j}\right) \in \mathbb{R}_{>0}$ that satisfies

$$
\psi(s):=s^{2}\left(\varphi(s)-Y_{\varepsilon}^{n, k}\left(p_{j}\right)\right)=0,
$$

where $\varphi(s):=s+\mu \phi^{+}(s)$ as in section 4 . To solve $\psi(s)=0$, we use Newton's method:

$$
s^{\ell+1}=s^{\ell}-\left[\psi^{\prime}\left(s^{\ell}\right)\right]^{-1} \psi\left(s^{\ell}\right), \quad \ell \geq 0,
$$

with $\left|s^{\ell+1}-s^{\ell}\right|<t o l$ as the stopping criterion and $s^{0}=U_{\varepsilon}^{n, k-\frac{3}{2}}\left(p_{j}\right)$ for $k \geq 2$ and $s^{0}=U_{\varepsilon}^{n, 0}\left(p_{j}\right)$ otherwise. Note that we introduced the term $s^{2}$ in (5.3) in order to stabilize the Newton iteration. Although other powers of $s$ are possible, this particular choice seemed preferable in practice. In fact, the iteration (5.4) always converged.

On the right-hand side of Figure 5, we plot $U_{\varepsilon}(x, T)$ and $V_{\varepsilon}(x, T)$ for $a=2 \times 10^{-3}$, $\delta=10^{-5}$, and $\nu=4$. The other parameters were chosen as follows: $c=10^{-5}$, $\rho=2 \times 10^{-3}$, initial data (ii) with $v_{\max }^{0}=1, \gamma=0, A=10, x_{0}=0.5, L=4$, $T=4.33$, and $\# J=2^{10}+1$. One can clearly see the effect of modeling the van der Waals forces. Once the film thickness reaches a certain threshold, the film tries to rupture in the effected regions. Note that we have plotted the solutions just before such a "rupture" occurs. Although the film height might become extremely thin, it can never actually rupture $\left(U_{\varepsilon}=0\right)$ due to the presence of the repulsive van der Waals forces. We would also like to mention that we repeated the experiment with the parameters mentioned above on a very fine mesh. As we obtained virtually identical results, we are satisfied that the oscillations shown in Figure 5 are not due to mesh effects. In fact, the instabilities are in agreement with linear stability analysis for the thin film equation in the presence of van der Waals forces (see [28, 27]).

5.2. Numerical results for $\boldsymbol{d}=\mathbf{2}$. Finally, we present numerical experiments in two space dimensions with $\Omega=(-L, L) \times(-L, L)$. We took a uniform mesh of squares $\varsigma$ of length $h=\frac{2 L}{128}$, each of which was divided into two triangles by its north 

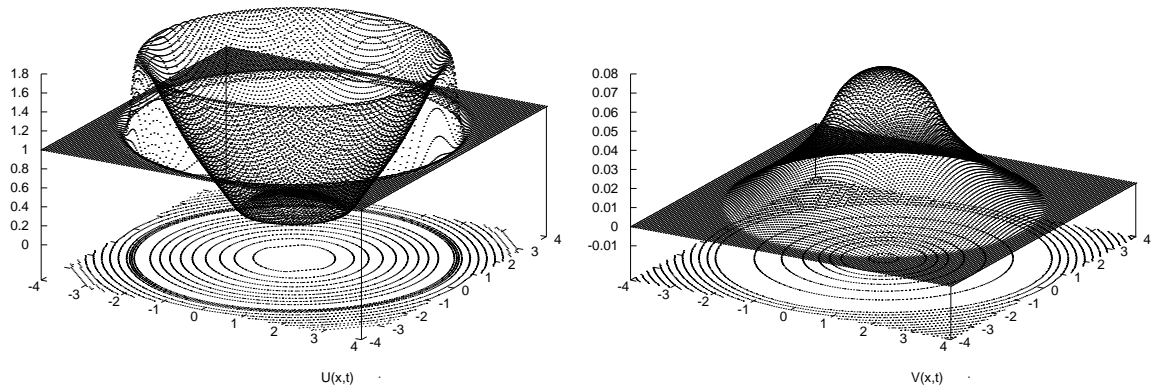

FIG. 6. $U_{\varepsilon}(x, T)$ and $V_{\varepsilon}(x, T)$ plotted against $x$ for $T=35$.

east diagonal. We used the modified discrete semi-inner product on $C(\bar{\Omega})$ :

$$
\left(\eta_{1}, \eta_{2}\right)_{*}^{h}:=\int_{\Omega} \Pi^{h}\left(\eta_{1}(x) \eta_{2}(x)\right) \mathrm{d} x .
$$

Here $\Pi^{h}$ is the piecewise continuous bilinear interpolant on $\bar{\Omega}$, which on each square $\varsigma$ is bilinear and interpolates at the vertices. Using (5.5) instead of (2.1) enables us to solve (4.6) efficiently using a "discrete cosine transform" approach; see [2]. We note that similarly to (2.1) and (2.14), the semi-inner product (5.5) is equivalent on $S^{h}$ to the standard $L^{2}$ inner product, and in place of (2.17), we have that

$$
\left|\left(z^{h}, \chi\right)^{h}-\left(z^{h}, \chi\right)_{*}^{h}\right| \leq C h^{1+m}\left[\ln \left(\frac{1}{h}\right)\right]^{2}\left\|z^{h}\right\|_{m}\left\|_{\chi}\right\|_{1} \quad \forall z^{h}, \chi \in S^{h}, \quad m=0 \text { or } 1 .
$$

Therefore, it is easy to adapt the proofs to show that all the results in this paper remain unchanged with the choice (5.5).

We report on an experiment with the same parameters for $(\mathrm{P})$ as in $d=1$ for Figure 4. In particular, we set $a=\delta=0, c=10^{-5}, \rho=2 \times 10^{-4}, L=4, T=35$, $\tau_{n}=\tau=10^{-3}$, and $\varepsilon=10^{-5}$, and for the initial profiles we chose $u^{0} \equiv 1$ and (5.1b) for $v^{0}$ with $v_{\max }^{0}=1, \gamma=0, A=10$, and $x_{0}=0.5$. We set $U_{\varepsilon}^{0} \equiv \pi^{h} u^{0}$ and $V_{\varepsilon}^{0} \equiv \pi^{h} v^{0}$. Note that $u^{0}, v^{0} \in W^{1, \infty}(\Omega)$, and hence the results of Lemma 2.5 still hold for the chosen $U_{\varepsilon}^{0}, V_{\varepsilon}^{0}$ on noting a standard interpolation result. Note, furthermore, that here we integrate until $T=35$ as opposed to $T=4$ in one space dimension. This is due to the slower speed of propagation; e.g., the corresponding similarity solution for $c=\rho=0$ advances proportionally to $(1+t)^{\frac{1}{4}}$ for $d=2$ as opposed to $(1+t)^{\frac{1}{3}}$ for $d=1$ (see [24]). We chose $t o l=10^{-8}$ and $\mu=400$ for the iterative method (4.2a-c). In Figure 6, we plot $U_{\varepsilon}(x, T)$ and $V_{\varepsilon}(x, T)$ for $T=35$, respectively.

\section{REFERENCES}

[1] R. A. Adams and J. Fournier, Cone conditions and properties of Sobolev spaces, J. Math. Anal. Appl., 61 (1977), pp. 713-734.

[2] J. W. BARRett And J. F. Blowey, Finite element approximation of a model for phase separation of a multi-component alloy with non-smooth free energy, Numer. Math., 77 (1997), pp. $1-34$.

[3] J. W. BARRett AND J. F. Blowey, Finite element approximation of a degenerate AllenCahn/Cahn-Hilliard system, SIAM J. Numer. Anal., 39 (2001), pp. 1598-1624.

[4] J. W. Barrett, J. F. Blowey, and H. Garcke, Finite element approximation of a fourth order nonlinear degenerate parabolic equation, Numer. Math., 80 (1998), pp. 525-556. 
[5] J. W. Barrett, J. F. Blowey, and H. Garcke, Finite element approximation of the CahnHilliard equation with degenerate mobility, SIAM J. Numer. Anal., 37 (1999), pp. 286-318.

[6] J. W. Barrett, J. F. Blowey, and H. Garcke, On fully practical finite element approximations of degenerate Cahn-Hilliard systems, M2AN Math. Model. Numer. Anal., 35 (2001), pp. $713-748$.

[7] J. W. BARRett AND R. NÜRnBerg, Finite element approximation of a nonlinear degenerate parabolic system describing bacterial pattern formation, Interfaces Free Bound., 4 (2002), pp. 277-307.

[8] F. BERNIS, Viscous flows, fourth order nonlinear degenerate parabolic equations and singular elliptic problems, in Free Boundary Problems: Theory and Applications, Pitman Res. Notes Math. Ser. 323, J. I. Diaz, M. A. Herrero, A. Linan, and J. L. Vazquez, eds., Longman, Harlow, UK, 1995, pp. 40-56.

[9] F. Bernis and A. Friedman, Higher order nonlinear degenerate parabolic equations, J. Differential Equations, 83 (1990), pp. 179-206.

[10] A. L. Bertozzi, M. P. Brenner, T. F. Dupont, and L. P. Kadanoff, Singularities and similarities in interface flows, in Trends and Perspectives in Applied Mathematics, Appl. Math. Sci. 100, Springer-Verlag, New York, 1994, pp. 155-208.

[11] M. Bertsch, R. Dal Passo, H. Garcke, and G. Grün, The thin viscous flow equation in higher space dimensions, Adv. Differential Equations, 3 (1998), pp. 417-440.

[12] J. F. Blowey And C. M. Elliott, The Cahn-Hilliard gradient theory for phase separation with nonsmooth free energy. II. Numerical analysis, European J. Appl. Math., 3 (1992), pp. 147-179.

[13] M. S. Borgas and J. B. Grotberg, Monolayer flow on a thin film, J. Fluid Mech., 193 (1988), pp. 151-170.

[14] J. W. Cahn, C. M. Elliott, and A. Novick-Cohen, The Cahn-Hilliard equation with a concentration dependent mobility: Motion by minus the Laplacian of the mean curvature, European J. Appl. Math., 7 (1996), pp. 287-301.

[15] J. F. Ciavaldini, Analyse numérique d'un problème de Stefan à deux phases par une méthode d'elements finis, SIAM J. Numer. Anal., 12 (1975), pp. 464-487.

[16] C. M. Elliott And H. Garcke, On the Cahn-Hilliard equation with degenerate mobility, SIAM J. Math. Anal., 27 (1996), pp. 404-423.

[17] C. M. Elliott and H. Garcke, Diffusional phase transitions in multicomponent systems with a concentration dependent mobility matrix, Phys. D, 109 (1997), pp. 242-256.

[18] D. P. Gaver III And J. B. Grotberg, The dynamics of a localized surfactant on a thin film, J. Fluid Mech., 213 (1990), pp. 127-148.

[19] H. P. Greenspan, On the motion of a small viscous droplet that wets a surface, J. Fluid Mech., 84 (1978), pp. 125-143.

[20] G. Grün, Degenerate parabolic differential equations of fourth order and a plasticity model with nonlocal hardening, Z. Anal. Anwendungen, 14 (1995), pp. 541-574.

[21] G. GRüN, On the convergence of entropy consistent schemes for lubrication type equations in multiple space dimensions, Math. Comp., 72 (2003), pp. 1251-1279.

[22] G. Grün And M. RumpF, Nonnegativity preserving numerical schemes for the thin film equation, Numer. Math., 87 (2000), pp. 113-152.

[23] G. GRün And M. RumpF, Simulation of singularities and instabilities in thin film flow, European J. Appl. Math., 12 (2001), pp. 293-320.

[24] O. E. Jensen And J. B. Grotberg, Insoluble surfactant spreading on a thin viscous film: Shock evolution and film rupture, J. Fluid Mech., 240 (1992), pp. 259-288.

[25] P. L. Lions AND B. Mercier, Splitting algorithms for the sum of two nonlinear operators, SIAM J. Numer. Anal., 16 (1979), pp. 964-979.

[26] O. K. Matar and S. M. Troian, The development of transient fingering patterns during the spreading of surfactant coated films, Phys. Fluids, 11 (1999), pp. 3232-3246.

[27] O. K. Matar and S. M. Troian, Spreading of a surfactant monolayer on a thin liquid film: Onset and evolution of digitated structures, Chaos, 9 (1999), pp. 141-153.

[28] A. Oron, S. H. Davis, And S. G. Bankoff, Long-scale evolution of thin liquid films, Rev. Modern Phys., 69 (1997), pp. 931-980.

[29] M. Renardy and R. C. Rogers, An Introduction to Partial Differential Equations, SpringerVerlag, New York, 1992.

[30] A. Schmidt And K. G. Siebert, Albert-software for scientific computations and applications, Acta Math. Univ. Comenian (N.S.), 70 (2001), pp. 105-122.

[31] S. Wieland, Modellierung und mathematische Analyse von Oberflächendiffusion auf dünnen Filmen, Ph.D. thesis, University of Bonn, Bonn, Germany, 2003 (to appear).

[32] L. Zhornitskaya AND A. L. BertozzI, Positivity-preserving numerical schemes for lubrication-type equations, SIAM J. Numer. Anal., 37 (2000), pp. 523-555. 